\title{
A Time Series Analysis-Based Stock Price Prediction Using Machine Learning and Deep Learning Models
}

\author{
Sidra Mehtab ${ }^{1}$ and Jaydip Sen ${ }^{2}$ \\ ${ }^{1}$ School of Computing and Analytics \\ NSHM Knowledge Campus, B. L. Saha Road \\ Kolkata 700104, West Bengal, INDIA \\ email: smehtab@acm.org \\ ${ }^{2}$ Departmnet of Data Science and Artificial Intelligence \\ Praxis Business School, Bakrahat Road, Off Diamond Harbor Road \\ Rasapunja, Kolkata - 7000104, West Bengal, INDIA \\ email: jaydip.sen@acm.org
}

\begin{abstract}
Prediction of future movement of stock prices has always been a challenging task for researchers. While the advocates of the efficient market hypothesis (EMH) believe that it is impossible to design any predictive framework that can accurately predict the movement of stock prices, there are seminal work in the literature that have demonstrated that the seemingly random movement patterns in the time series of a stock price can be predicted with a high level of accuracy. The design of such predictive models requires the choice of appropriate variables, right transformation methods of the variables, and tuning of the parameters of the models. In this paper, we present a very robust and accurate framework of stock price prediction that consists of an agglomeration of statistical, machine learning, and deep learning models. We use daily stock price data, collected at five minutes intervals of time, of a very well-known company that is listed in the National Stock Exchange (NSE) of India. The granular data is aggregated into three slots in a day, and the aggregated data is used for training and building the forecasting models. We contend that the agglomerative approach of model building that uses a combination of statistical, machine learning, and deep learning approaches, can very effectively learn from the volatile and random movement patterns in stock price data. This effective learning will lead to the building of very robust training of the models that can be deployed for short-term forecasting of stock prices, and prediction of stock movement patterns. We build eight classification and eight regression models based on statistical and machine learning approaches. In addition to these models, two deep learning-based regression models using a long-and-short-term memory (LSTM) network and a convolutional neural network $(\mathrm{CNN})$ have also been built. Extensive results have been presented on the performance of these models, and results are critically analyzed.
\end{abstract}

\section{Introduction}

Prediction of future movement patterns of stock prices has been a widely researched area in the literature. While there are proponents of the efficient market hypothesis who believe that it is impossible to predict stock prices, there are also propositions that demonstrated that if correctly formulated and modeled, the prediction of stock prices can be done with a fairly high level of accuracy. The latter school of thought focused on the construction of robust statistical, econometric, and machine learning models based on the careful choice of variables and appropriate functional forms or models of forecasting. There are 
propositions in the literature that are based on time series analysis and decomposition for forecasting future values of stocks. In this regard, several propositions have been presented in the literature for stock price forecasting following a time series decomposition approach. (Sen \& Datta Chaudhuri, 2018a; Sen, 2018b; Sen, 2018c; Sen \& Datta Chaudhuri, 2016a; Sen \& Datta Chaudhuri, 2016b). There is also an extent of literature that deals with various technical analysis of stock price movements. Propositions also exist for mining sock price patterns using various important indicators like Bollinger Bands, moving average convergence divergence (MACD), relative strength index (RSI), moving average (MA), stochastic momentum index (SMI), etc. There are also well-known patterns like head and shoulders pattern, inverse head and shoulders pattern, triangle, flag, Fibonacci fan, Andrew's Pitchfork, etc., which are exploited by traders for investing intelligently in the stock market. These approaches provide the user with visual manifestations of the indicators which help the ordinary investors to understand which way stock prices are more likely to move soon. In this thesis, we propose a granular approach to forecasting stock price and the

price movement pattern by combining several statistical, machine learning, and deep learning methods of prediction on technical analysis of stock prices. We present several approaches for short-term stock price movement forecasting using various classification and regression techniques and compare their performance in the prediction of stock price movement and stock price values. We believe this approach will provide several useful information to the investors in the stock market who are particularly interested in short-term investments for profit. This work is a modified and extended version of our previous work (Mehtab \& Sen, 2019). In the present work, we have presented a predictive framework that aggregates eight classification and eight regression models including a long-and short-term memory (LSTM)-based advanced deep learning model, and four variants of convolutional neural network (CNN)-based forecasting models.

The objective of our work is to take stock price data at five minutes intervals from the National Stock Exchange (NSE) of India and develop a robust forecasting framework for the stock price movement. We contend that such a granular approach can model the inherent dynamics and can be fine-tuned for immediate forecasting of stock price or stock price movement. Here, we are not addressing the problem of forecasting of long-term movement of the stock price. Rather, our framework will be more relevant to a trade-oriented framework.

The rest of the paper is organized as follows. Section 2 presents a comprehensive review of the literature on stock price movement modeling and prediction. In Section 3, we present a detailed discussion on the methodology that we have followed in this work. Section 4 provides a brief discussion on the working principles of the classification and the regression models in machine learning that we have used in this work. In Section 5, we present a summary of two deep learning-based models - LSTM-based deep learning model for regression and four variants of $\mathrm{CNN}$-based forecasting models - that we have also used in our predictive model. Section 6 presents a detailed discussion on the performance of machine learning and deep learning models. A comparative analysis of the performances of the models is also presented in this section. Finally, Section 7 concludes the paper.

\section{Related Work}

The literature attempting to prove or disprove the efficient market hypothesis can be classified into three strands, according to the choice of variables and techniques of estimation and forecasting. The first strand consists of studies using simple regression techniques on cross-sectional data (Enke et al., 2011; Ma \& Liu, 
2008; Khan et al., 2018; Ivanovski, 2016; Sen \& Datta Chaudhuri, T, 2016c. The second strand of the literature has used time series models and techniques to forecast stock returns following economic tools like autoregressive integrated moving average (ARIMA), Granger causality test, autoregressive distributed lag (ARDL), and quantile regression (QR) to forecast stock prices (Ariyo et al., 2014; Jammalamadaka et al., 2019; Jarrett \& Kyper, 2011; Mondal et al., 2014; Sen \& Datta Chaudhuri, 2017; Xiao et al., 2014). The third strand includes work using machine learning, deep learning, and natural language processing for the prediction of stock returns (Mostafa, 2010; Dutta et al., 2006; Mehtab \& Sen, 2019; Mehtab \& Sen, 2020a; Mehtab \& Sen, 2020b; Mehtab \& Sen, 2020c; Mehtab et al., 2020d; Mehtab et al., 2020e; Mehtab \& Sen, 2021; Porshnev et al., 2013; Obthong et al., 2020; Sen, 2018d; Tang \& Chen, 2018; Wang et al., 2018; Zhou \& Fan, 2019; Wu et al., 2008).

Among some of the recent propositions in the literature on stock price prediction, Mehtab and Sen have demonstrated how machine learning and long-and short-term memory (LSTM)-based deep learning networks can be used for accurately forecasting NIFTY 50 stock price movements in the National Stock Exchange (NSE) of India (Mehtab \& Sen, 2019). The authors used the daily stock prices for three years from January 2015 till December 2017 for building the predictive models. The forecast accuracies of the models were then evaluated based on their ability to predict the movement patterns of the close value of the NIFTY index on a time horizon of one week. For testing, the authors used NIFTY 50 index values for January 2018 till June 2019. To further improve the predictive power of the models, the authors incorporated a sentiment analysis module for analyzing the public sentiments on Twitter on NIFTY 50 stocks. The output of the sentiment analysis module is fed into the predictive model in addition to the past NIFTY 50 index values for building a very robust and accurate forecasting model. The sentiment analysis module uses a self-organizing fuzzy neural network (SOFNN) for handling non-linearity in a multivariate predictive environment.

Mehtab and Sen recently proposed another approach to stock price and movement prediction using convolutional neural networks (CNN) on a multivariate time series (Mehtab \& Sen, 2020). The predictive model proposed by the authors exploits the learning ability of a CNN with a walk-forward validation ability to realize a high level of accuracy in forecasting the future NIFTY index values, and their movement patterns. Three different architectures of CNN are proposed by the authors that differ in the number of variables used in forecasting, the number of sub-models used in the overall system, and the size of the input data for training the models. The experimental results indicated that the CNN-based multivariate forecasting model was highly accurate in predicting the movement of NIFTY index values with a weekly forecast horizon. Use of LSTM networks in stock price prediction has also been proposed (Sen et al., 2021a; Sen et al., 2021b).

Zhang et al. proposed the application of a multilayer backpropagation (BP) neural network in financial data mining (Zhang et al., 2007). The proposed scheme was a modified neural network-based forecasting model that carries out intelligent mining tasks. The system was capable of making robust forecasting on the buying and selling signs according to the prediction of future trends in the stock market. The simulation results on seven years of data of the Shanghai composite index indicated that the return achieved by the system is about three times that achieved by the buy-and-hold strategy.

Basalto et al. proposed an approach based on a pair-wise clustering to analyze the Dow Jones Index companies to identify similar temporal behavior of the traded stock prices (Basalto et al., 2005). The main goal of the authors was to investigate and understand the dynamics that govern companies' stock prices. The proposed scheme deployed a pairwise version of the chaotic map algorithm that was executed based 
on correlation coefficients between the financial time series to find similarity measures for clustering the temporal patterns. The resultant dynamics of such systems formed the clusters of companies that belong to different industrial branches. These clusters of companies can be gainfully exploited to optimize portfolio construction.

Chen et al. have proposed an approach for constructing a model for predicting the direction of return on the Taiwan Stock Exchange Index (Chen et al., 2003). The authors contended that the stock trading guided by robust forecasting models were more effective and usually led to a higher return on investment. To construct a robust forecasting model, the authors built and trained a probabilistic neural network (PNN) using historical stock market data. The forecasted output of the model was applied to form various index trading strategies, and the effectiveness of those strategies was compared with those generated by the buy and hold strategy, the investment strategies formed using the output of a random walk model, and the parametric generalized method of moments (GMM) with a Kalman filter. The results showed that the investment strategies made using the output of the PPN yielded the highest return of investment in the long-run.

de Faria et al. illustrated a predictive model using a neural network and an adaptive exponential smoothing (AES) method for forecasting the movements of the principal index of the Brazilian stock market (de Faria et al., 2009). The authors compared the forecasting performance of both the neural network and the exponential smoothing models with a particular focus on the sign of the market returns. While the simulation results showed that both methods were equally efficient in predicting the index returns, the neural network model was found to be more accurate in predicting the market movement than the adaptive exponential smoothing method.

Leigh et al. proposed the use of linear regression and simple neural network models for forecasting the stock market indices in the New York Stock Exchange during the period 1981-1999 (Leigh et al., 2005). The proposed scheme by the authors used a template matching mechanism based on statistical pattern recognition that efficiently and accurately identified spikes in the trading volumes. A threshold limit for the spike in volume was identified, and the days on which the traded volume exhibited significant spikes were identified. A linear regression model was applied to forecast the future change in price based on the historical price, traded volume, and the prime interest rate.

Shen et al. proposed a novel scheme that was based on a tapped delay neural network (TDNN) with an ability of adaptive learning and pruning for forecasting on a non-linear time series of stock price values (Shen et al., 2007). The TDDN model was trained by a recursive least square (RLS) technique that involved a tunable learning-rate parameter that enables faster network convergence. The trained neural network model was optimized using a pruning algorithm that reduced the possibility of overfitting of the model. The experimental results in a simulated environment clearly showed that the pruned model had a reduced complexity, faster execution, and improved prediction accuracy.

Ning et al. proposed a scheme of stock index prediction that was based on a chaotic neural network (Ning et al., 2009). Data from a Chinese stock market and a Shenzhen stock market were used for building the model. The non-linear, stochastic, and chaotic patterns in the stock market indices were learned by the chaotic neural network, and the learnings of the chaotic neural network were gainfully applied in forecasting future index values of the stock markets.

Hanias et al. conducted a study to predict the daily stock exchange price index of the Athens Stock Exchange (ASE) using a neural network with backpropagation (Hanias et al., 2012). The neural network 
was used to make a multistep forecasting for nine days and yielded a very low mean square error (MSE) value of 0.0024 .

$\mathrm{Wu}$ et al. proposed an ensemble model of prediction using support vector machines (SVM) and artificial neural networks (ANN) for predicting stock prices (Wu et al., 2008). The forecasting performance of the ensemble model was compared with those of the SVM model and the ANN model. It was observed by the authors that the ensemble approach produced more accurate results than the other two models.

Liao et al. carried out a study on the stock market investment issues on the Taiwan stock market (Liao et al., 2008). The scheme involved two phases. In the first phase, the apriori algorithm was used to identify the association rules and knowledge patterns about stock category association and possible stock category investment collections. After the association rules were successfully mined, in the second phase, the kmeans clustering algorithm was used to identify the various clusters of stocks based on their association patterns. The authors also proposed several possible stock market portfolio alternatives under various clusters of stocks.

Zhu et al. hypothesized that there is a significant bidirectional nonlinear causality between stock returns and trading volumes (Zhu et al., 2008). The authors proposed the use of a neural network-based scheme for forecasting stock index movements. The model was further enriched by the inclusion of different combinations of indices and component stocks' trading volumes as inputs. NASDAQ, DJIA, and STI data of stock prices and volume of transactions were used in training the neural network. The experimental results demonstrated that the augmented neural networks with trading volumes lead to improvements in forecasting performance under different terms of the forecasting horizon.

Bentes et al. presented a study on the long memory and volatility clustering for the S\&P 500, NASDAQ 100, and Stoxx 50 indexes to compare the US and European markets (Bentes et al., 2008). The authors compared the performance of two different approaches. The first approach was based on the traditional approaches using generalized autoregressive conditional heteroscedasticity $\operatorname{GARCH}(1,1), \operatorname{IGARCH}(1,1)$, and FIGARCH (1, d, 1), while the second approach exploited the concept of entropy in the Econophysics. In the second approach, three different measures were considered by the authors in the study. The three measures were Shannon, Renyi, and Tsallis measures. The results obtained using both the approaches elicited the existence of nonlinearity and volatility of SP 500, NASDAQ 100, and Stoxx 50 indexes.

Chen et al. demonstrated how the random and chaotic behavior of stock price movements can be very effectively modeled using a local linear wavelet neural network (LLWNN) technique (Chen et al., 2005). The proposed wavelet-based model was further optimized using a novel algorithm, which the authors referred to as estimation of distribution algorithm (EDA). The purpose of the model was to accurately predict the share price for the following trade day given the opening, closing, and maximum values of the stock price for a particular day. The study revealed an interesting observation - even for a time series that exhibited an extremely high level of random fluctuations in its values, the model could extract some very important features from the opening, closing, and the maximum values of the stock index that enabled an accurate prediction of its future behavior.

Dutta et al. illustrated how ANN models could be applied in forecasting the Bombay Stock Exchange's SENSEX weekly closing values from January 2002 to December 2003 (Dutta et al., 2006). The proposed approach by the author involved building two neural networks each consisting of three hidden layers, in addition to the input and the output layers. The input values to the first neural network were: (i) the weekly closing values, (ii) the 52-week moving average of the weekly closing SENSEX values, (iii) the 5-week 
moving average of the closing values, and (iv) the 10-week oscillator values for the past 200 weeks. On the other hand, the second network was provided with the following input values: (i) weekly closing value of SENSEX, (ii) the moving average of the weekly closing values computed on the 52-week historical data, (iii) the moving average of the closing values computed on the 5 -week historical data, and (iv) the volatility of the SENSEX records computed on 5-week basis over the past 200 weeks. The forecasting performance of the two neural networks was compared using their root mean square error (RMSE) and mean absolute error (MSE) values on the test data. To test the networks, the weekly closing SENSEX values for the period of January 2002 to December 2003 were used.

Hammad et al. demonstrated that an artificial neural network (ANN) model can be trained to converge to an optimal solution while it maintains a very high level of precision in the forecasting of stock prices (Hammad et al., 2009). The proposed scheme was based on a multi-layer feedforward neural network model that used the back-propagation algorithm. The model was used for forecasting the Jordanian stock prices. The authors demonstrated simulations using MATLAB that were carried on seven Jordanian companies from the service and manufacturing sectors. The accuracy of the model in forecasting stock price movement was found to be very high.

Tseng et al. utilized various approaches including the traditional time series decomposition (TSD) model, HoltWinters $(\mathrm{H} / \mathrm{W})$ exponential smoothing with trend and seasonality models, Box-Jenkins (B/J) models using autocorrelation and partial autocorrelation, and neural network-based models (Tseng et al, 2012). The authors trained the models on the stock price data of 50 randomly chosen stocks during the period: September 1, 1998 - December 31, 2010. To train the models, 3105 observations based on closed prices of the stocks were used. The testing of the model was carried out on data spanning over 60 trading days. The study showed that the forecasting accuracies were higher for $\mathrm{B} / \mathrm{J}, \mathrm{H} / \mathrm{W}$, and normalized neural network models. The errors associated with the time series decomposition-based model and the non-normalized neural network models were found to be higher.

Senol and Ozturan illustrated that ANN can be used to predict stock prices and their direction of changes (Senol \& Ozturan, 2008). The result was promising with a forecast accuracy of $81 \%$ on average.

Fu et al. presented an approach that represented the data points in a financial time series according to their importance (Fu et al., 2008). Using the ranked data points based on their importance, a tree was constructed that enabled incremental updating of data in the time series. The scheme facilitated the representation of a large-sized time series in different levels of details, and also enabled multi-resolution dimensionality reduction. The authors have presented several evaluation methods of data point importance, a novel method of updating a time series, and two-dimensionality reduction approaches. Extensive experimental results are also presented demonstrating the effectiveness of all propositions.

Phua et al. presented a predictive model using neural networks with genetic algorithms for forecasting stock price movements in the Singapore Stock Exchange (Phua et al., 2001). The forecasting accuracy of the predictive model was found to be $81 \%$ on the test dataset indicating that the model was moderately effective in its forecasting job.

Moshiri and Cameron described a back propagation-based neural network and a set of econometric models to forecast inflation levels (Moshiri, \& Cameron, 2010). The set of econometric models proposed by the authors included the following: (i) Box-Jenkins autoregressive integrated moving average (ARIMA) model, (ii) vector autoregression (VAR) model, and (iii) Bayesian vector autoregression (BVAR) model. The forecasting accuracies of the three models were compared with the hybrid back propagation network (BPN) 
model proposed by the authors. To test the models, three different values of the forecasting horizon were used: one month, two months, and twelve months. With the root mean square error (RMSE) and the mean absolute error (MAE) as the two metrics, the authors observed that the performance of the hybrid BPN was superior to the other econometric models.

Asghar et al. contend that the stock price data acquired from social media and the websites storing financial data, is usually sparse, and often the predictors used for building the stock price prediction models are poorly chosen (Asghar et al., 2019). This leads to poor performance of the models. The authors propose a multiple regression model that systematically selects the predictors, and hence yields a high accuracy in forecasting. The system also has a user-friendly interface that provides a rich user experience. However, the model is too simple to effectively handle time series containing highly volatile stock prices.

Park et al. present a model for stock price prediction that is based on multiple regression (Park et al., 2010). The model has the capability of estimating the risk associated with a stock price. However, the framework is too simple and the assumptions made in designing the model may not be holding good in real-world times series of volatile stock price movements.

Yan et al. propose a hybrid predictive model that consists of a multiple linear regression model and a backpropagation (BP) neural network model for predicting the movements of the stock prices (Yan et al., 2019). The results make it evident that the BP neural network is more accurate than its multiple regression counterpart. However, the effectiveness of the BP neural network model on a highly granular and volatile stock price data is questionable.

Vantuch et al. propose an ARIMA-based stock price forecasting model (Vantuch \& Zelinka, 2014). The ARIMA model is further fine-tuned using evolutionary algorithms that are based on genetic algorithms (GA) and particle swarm optimization (PSO). The model is found to have a very high level of accuracy in forecasting. However, its applicability on a very highly granular time series data with a very short forecast horizon is not addressed by the authors.

Ning et al. investigate the relationship between several macroeconomic variables, e.g., interest rate, money supply, exchange rate, inflation rate, etc., and their effect on stock returns in Hong Kong and Shanghai (Ning et al., 2019). The relationships are tested using arbitrage pricing theory (APT), vector error correction model (VECM), and the Granger causality test. The results elicit an important observation investors should have long-term investments in the Chinese stock market for getting a good return on their investments, while for the Hong Kong stock markets, the case is just the opposite.

Bao et al. present a hybrid deep learning framework for stock price prediction that consists of three components: (i) wavelet transform (WT), (ii) stacked autoencoders (SAEs), and (iii) long-and short-term memory (LSTM) gates (Bao et al., 2017). Initially, the time series of the stock price data is decomposed the WT for denoising of the data. The denoised data is passed on to the SAEs that extract deep features from the data, which are, then, passed into the LSTM module for predicting the future stock prices. The model is found to yield very high accuracy. However, the model is evaluated on a stock price data that has a daily frequency. Hence, it is not suitable for intra-day investment decisions.

Vargas et al. propose a sophisticated deep learning model for the detection and analysis of complex patterns and interactions among stock price data (Vargas et al., 2017). The deep learning framework consists of a $\mathrm{CNN}$ and a RNN model. The results show that $\mathrm{CNN}$ is more effective in catching the semantic meaning 
from the text inputs to the model, while RNN is superior in understanding the context information and modeling the temporal characteristics for the stock price prediction.

The major drawback of the existing propositions in literature for stock price prediction is their inability to predict stock price movement in a short-term interval. The current work attempts to address this shortcoming by exploiting the learning ability of a gamut of machine learning and two deep neural networks in stock price movement modeling and prediction.

\section{Methodology}

In Section 1, we mentioned that the goal of this work is to develop a robust forecasting framework for the short-term price movement of stocks. We use the Metastock tool for collecting data on the short-term price movement of stocks (Metastock). Particularly, we collected the stock data for the company - Godrej Consumer Products Ltd. The data is collected at every 5 minutes interval in a day, for all the days in which the National Stock Exchange (NSE) was operational during the years 2013 and 2014. The raw data for each stock consisted of the following variables: (i) date, (ii) time, (iii) open value of the stock, (iv) high value of the stock, (v) low value of the stock, (vi) close value of the stock, and (vii) volume of the stock traded in a given interval. The variable time refers to the time instance at which the stock values are noted as each record is collected at 5 minutes interval of time. Hence, the time interval between two successive records in the raw data was 5 minutes. The raw data in this format is collected for the stock Godrej Consumer Products. for two years. In addition to the seven variables in the raw data that we have mentioned above, we also collected the NIFTY index at 5 minutes interval for the same period of two years, To capture the overall market sentiment at each time instant, so that more accurate and robust forecasting can be made using the combined information of historical stock prices and the market sentiment index. Therefore, the raw data for both the stocks now consists of seven variables. As 5 minutes interval is too granular, we make some aggregation of the raw data. We break the total time interval in a day into three slots as follows: (1) morning slot that covers the time interval 9:00 AM till 11:30 AM, (2) afternoon slot that covers the time interval 11:35 AM till 1:30 PM, and (3) evening slot that covers the time interval 1:35 PM till the time of closure of NSE in a given day. Hence, the daily stock information now consists of three records, each record containing stock price information for a time slot.

Using the eight variables in the raw data, and incorporating the aggregation of data using the time slots, we create eleven derived variables and compute their values. These derived variables are used as the input variables for building the predictive models for forecasting the stock price and the stock movement. We followed two approaches to stock price forecasting - regression and classification. The difference in these two approaches lied in the way the response variable open_perc was used in the model building process. This point will be described in detail later in this Section.

The following are the eleven variables that are computed for designing the machine learning models.

month: This is a numeric variable that refers to the month for a given stock price record. The twelve months are assigned numeric codes of 1 through 12, with January being coded as 1, and the month of December assigned with a code of 12 .

day_month: This numeric variable denotes the particular day of a given month to which a stock price record corresponds. The value of this variable lies in the interval [1,31]. For instance, if the date for a stock price record is 22nd May 2013 then the day_month variable for that record will be assigned a value of 22 . 
day_week: This is a numeric variable that corresponds to the day of the week for a given stock price record. The five days in a week on which the stock market remain open are assigned numeric codes of 1 through 5, with Monday being coded as 1, while Friday is assigned a code of 5.

time: This numeric variable refers to the time slot to which a stock price record belongs. There are threetime slots in a day - morning, afternoon, and evening. The slots are assigned codes 1, 2, and 3 respectively. For example, if a stock price record refers to the time point 3:45 PM, the variable time will be assigned a value of 3 for the stock price record.

open_perc: it is a numeric variable that is computed as a percentage change in the value of the open value of the stock over two successive time slots. The computation of the variable is done as follows. Suppose, we have two successive slots: $S_{1}$ and $S_{2}$. Both of them consist of several records at five minutes intervals of time. Let the open price of the stock for the first record of $S_{1}$ is $X_{1}$ and that for $S_{2}$ is $X_{2}$. The open_perc for the slot $S_{2}$ is computed as $\left(X_{z}-X_{1}\right) / X_{2}$ in terms of percentage.

high_perc: it is a numeric value that is computed as the difference between the high values of two successive slots. The computation is identical to that of open_perc except for the fact that high values are used in this case instead of the open values.

low_perc: it is a numeric value that is computed as the difference between the low values of two successive slots. For two successive slots $\mathrm{S}_{1}$ and $\mathrm{S}_{2}$, first we compute the mean of all low values of the records in both the slots. If $L_{1}$ and $L_{2}$ refer to the mean of the low values for $S_{1}$ and $S_{2}$ respectively, then low perc for $S_{2}$ is computed as $\left(L_{2}-L_{1}\right) / L_{2}$ in terms of percentage.

close perc: it is a numeric value that is computed as the difference between the close values of two successive slots. Its computation is similar to the open_perc variable, except for the fact that we use the close values in the slots instead of the open values.

vol perc: it is a numeric value that is computed as the difference between the volume values of two successive slots. For two successive slots $S_{1}$ and $S_{2}$, we compute the mean values of volume for both the slots, say $V_{1}$ and $V_{2}$ respectively. Now, the vol_perc for $S_{2}$ is computed as $\left(V_{2}-V_{1}\right) / V_{2}$ in terms of percentage.

nifty_perc: it is a numeric variable that is computed as a percentage change in the NIFTY index over two successive time slots. The computation of the variable is done as follows. We compute the means of the NIFTY index values for two successive time slots $S_{1}$ and $S_{2}$. Let us assume the means are $M_{1}$ and $M_{2}$ respectively. Then the nifty perc for the slot $S_{2}$ is computed as $\left(M_{2}-M_{1}\right) / M_{2}$ in terms of percentage.

range_diff: The value of this numeric variable is obtained by computing the difference in the range values of two consecutive time slots. The range value for a given slot is the difference between its high and low values. If $S_{1}$ and $S_{2}$, denote two consecutive slots, and if $H_{1}, H_{2}, L_{1}$, and $L_{2}$ respectively represent the high and the low values of the slots $S_{1}$ and $S_{2}$, then the range value for $S_{1}$ is $R_{1}=\left(H_{1}-L_{1}\right)$ and for $S_{2}$ is $R_{2}=\left(H_{2}\right.$ - $\left.L_{2}\right)$. The range_diff for the slot $S_{2}$ is computed as $\left(R_{2}-R_{1}\right)$.

After we compute the values of the above eleven variables for each slot for both the stocks for the time frame of two years (i.e., 2013 and 2014), we develop the forecasting framework. As mentioned earlier, we followed two broad approaches in the forecasting of the stock prices - regression and classification.

In the regression approach, based on the historical movement of the stock prices we predict the stock price in the next slot. We use open_perc as the response variable, which is a continuous numeric variable. The objective of the regression technique is to predict the open_perc value of the next slot, given the stock 
movement pattern and the values of the predictors till the previous slot. In other words, if the current time slot is $S_{1}$, the regression techniques will attempt to predict open_perc for the next slot $S_{2}$. If the predicted open_perc is positive, then it will indicate that there is an expected rise in the stock price in $S_{2}$, while a negative open_perc will indicate a fall in the stock price in the next slot. Based on the predicted values, a potential investor can make his/her investment decision in stocks.

In the classification approach, the response variable open_perc is a discrete variable belonging to one of the two classes - " 0 " or " 1 ". For designing the classification-based forecasting models, we converted open_perc into a categorical variable that assumes one of the two values " 0 " and " 1 ". While " 0 " indicates a negative or a zero open_perc value, " 1 " indicates a positive open_perc value. Hence, if the current slot is $S_{1}$ and if the predictive model expects a rise in the open_perc value in the next slot $S_{2}$, then the open_perc value for $S_{2}$ will be "1". An expected negative value of the open_perc in the next slot will be indicated by a " 0 " value for the response variable.

For both classification and regression approaches, we studied three cases. In the following, these scenarios are described in detail.

Case I: We used the data for the year 2013 which consisted of 19, 385 records at five minutes intervals. These records were aggregated into 745-time slot records for building the predictive model. We used the same dataset for testing the forecast accuracy of the models for the stock of Godrej Consumer Products Ltd. and carried out a comparative analysis of all the models.

Case II: We used the data for the year 2014 which consisted of 18, 972 records at five minutes intervals. These granular data were aggregated into a 725 -time slot record for building the predictive model. We used the same dataset for testing the forecast accuracy of the models and carried out an analysis of the performance of the predictive models.

Case III: We used that data for 2013 as the training dataset for building the models and test the models using the data for the year 2014 as the test dataset. We, again, carried out an analysis of the performance of different models in this approach.

We build eight classification models and ten regression models for developing our forecasting framework. The classification models are: (i) logistic regression, (ii) $k$-nearest neighbor (iii) decision tree, (iv) bagging, (v) boosting, (vi) random forest, (vii) artificial neural network, and (viii) support vector machines. For measuring accuracy and effectiveness in these approaches, we use several metrics such as sensitivity, specificity, positive predictive value, negative predictive value, classification accuracy, and F1 score. Sensitivity and positive predictive value are also known as recall and precision respectively.

The ten regression methods that we build are: (i) multivariate regression, (ii) multivariate adaptive regression spline (MARS), (iii) decision tree, (iv) bagging, (v) boosting, (vi) random forest, (vii) artificial neural network, (viii) support vector machine, (ix) long-and short-term memory (LSTM) network, (x) convolutional neural network (CNN).

While all the classification techniques are machine learning-based approaches, two regression techniques, i.e., long-and short-term memory (LSTM) network, and convolutional neural network (CNN) - based approaches are deep learning methods. For comparing the performance of the regression methods, we use three metrics: (i) root mean square error (RMSE), (ii) correlation coefficient between the actual and predicted values of the response variable, e.g., open_perc, and (iii) the number of cases in which the predicted and the actual values of open_perc differed in their signs. 


\section{Machine Learning Models}

The eight classification models that we built are discussed in detail in this Section.

Logistic Regression: Logistic regression is a classification technique, and hence in building this model, we transform the response variable open_perc into a discrete (i.e., categorical) variable that can assume two discrete values - " 0 " or " 1 ". We convert all negative or zero values of open_perc to the class " 0 ", and all positive values to class " 1 ". We use the function $g l m$ in R for building the logistic regression model with three parameters passed into the function: (i) the first parameter is the formula which is: open_perc $\sim$. to include open_perc as the response variable and all the remaining variables as the predictors, (ii) the second parameter is: family = binomial - this indicates that model is a binary logistic regression involving two classes, and (iii) the third parameter is the $\mathrm{R}$ data object containing the training data set. We use the predict function in $\mathrm{R}$ to compute the probabilities of the test records belonging to the two classes. A threshold probability value of 0.5 is assumed. In other words, when the probability of a record belonging to a class exceeds 0.5 , the record is classified into that particular class.

K-Nearest Neighbor: The KNN model follows what is known as instance-based learning. Based on the training, the classification for a new unclassified record is found simply by comparing it to the most similar records in the training set. The value of $k$ determines how many closest similar records in the training data set is considered for classifying a test data set record. We use the function knn defined in the R library class to carryout KNN classification in the stock price data. The data is normalized using min-max normalization before applying the knn function so that all predictors are scaled down into the same range of values. Different values of $k$ are tried out for building the models, and the value of $k=3$ is finally chosen. This value of $k$ is found to yield the best performance with the minimum possibility of model overfitting.

Decision Tree: The classification and regression tree (CART) algorithm produces decision trees that are strictly binary so that there are exactly two branches at each node. The algorithm recursively partitions the records in the training data set into subsets of records with similar values for the target attributes. The trees are constructed by carrying out an exhaustive search on each node for all available variables and all possible splitting values and selects the optimal split based on some goodness of split criteria. We use the tree function defined in the tree library of $\mathrm{R}$ for the classification of the stock records.

Bagging: Bootstrap Aggregation (Bagging) is an ensemble technique. It works as follows: Given a set $D$, of $d$ tuples, for iteration $i$, a training set $D_{i}$ of $d$ tuples is sampled with replacement from the original set of $D$ tuples. Each training set represents a bootstrap sample. Since the samples are simple random samples with replacement, some records (i.e., tuples) in $D$ may not get a chance to be included in $D_{i}$, while some tuples may get included in more than one sample. A classifier model $M_{i}$ is trained on the information contained in each training set $D_{i}$. For classifying an unknown tuple $X$ in the out-of-sample set (i.e., in the test dataset), each classifier $M_{i}$ is asked to return its class predictions. The classification result of each of the trained classifier is considered as one vote. The bagging classifier counts the votes and finally assigns the class with the maximum number of votes to the tuple $X$. For carrying out classification on stock price data, we use bagging function defined in the ipred library of $\mathrm{R}$. The value of the parameter $n b a g$ is taken as 25. This specifies the number of bagging classifiers used in simple majority voting.

Boosting: Unlike bagging, boosting assigns weights to each tuple in a training dataset. Based on the training dataset, $k$ classification models are built iteratively. However, all the classifiers are not given equal 
importance in the final classification decision. Unlike bagging which uses simple majority voting among the classifiers, boosting uses a weighted majority voting mechanism. After a classifier $M_{i}$ is constructed, the weights assigned to the classifiers are updated before building the subsequent classifier $M_{i+1}$. After the completion of the current iteration, the classifiers that could correctly classify the tuples which were misclassified in the previous round are assigned higher weights before the next iteration of classifier construction starts. After the completion of the final round, the boosted classifier model combines the weighted votes of each classifier, where the weights are computed based on some functions of the classification accuracies of the results reported by the individual classifier. Adaptive Boosting (AdaBoost) is a very popular variant of boosting, and for the classification purpose, we have used this approach. The boosting function of the adabag library in $\mathrm{R}$ is used in the implementation.

Random Forest: Random forest is an ensemble machine learning approach. The algorithm first builds a large number of decision tree classifiers separately so that the collection of the classifiers is a forest. The individual decision tree classifier models are built based on a random selection of attributes at each node. The splitting at each node is done by randomly selecting the feature and the feature value for splitting to introduce as much randomness as possible. In other words, each decision tree depends on the values of a random vector sampled independently, and with the same distribution for all trees in the forest. The objective of introducing so much randomness in building the decision tree models is to avoid overfitting of the models during the training phase. During the classification phase, each tree votes, and the test case is assigned the class that is returned by the majority of the trees. We use the randomForest function defined in the randomForest library in $\mathrm{R}$ in our implementation.

Artificial Neural Network: An artificial neural network (ANN) is a connectionist network that consists of nodes and their interconnecting links where the nodes are arranged in several layers - an input layer, one or more hidden layers, and an output layer. The nodes in the input layer correspond to the predictor variables (i.e., attributes) in the training dataset. The inputs are fed simultaneously into the units making up the input layer. The input values pass through the respective nodes in the input layer and are then weighted using the weights associated with the links connecting the nodes and fed simultaneously to the second layer of nodes, known as the hidden layer nodes. The outputs of the nodes in the first hidden layer are weighted again using the corresponding link weights, and the resultant values are provided as the inputs to a possible second hidden layer and so on. The weighted outputs of the last hidden layer are input to units making up the output layer, which produces the network's prediction for given tuples. We use the neuralnet function defined in the neuralnet library in R for implementing the ANN classifier. The raw data is normalized using the minmax normalization approach. Only the predictors are normalized, the response variable: open_perc is kept unchanged. The parameter hidden of the function neuralnet is varied to change the number of nodes in the hidden layer. The parameter stepmax is set to the maximum value of $10^{6}$ so that the maximum number of iteration capability of the neuralnet function can be utilized. For implementing a classification model, the parameter linear.output is set to FALSE in the neuralnet function.

Support Vector Machine: A support vector machine (SVM) model, when applied for classification, can classify both linear and nonlinear data. For linearly inseparable data, it uses a nonlinear mapping to transform the original data into a higher dimension. Within this new higher dimension, it searches for the linear optimal hyperplane that separates the two classes. SVM finds this hyperplane using support vectors which are the essential and the discriminating training tuples to separate the two classes. We use the $\mathrm{ksvm}$ function defined in the kernlab library in R for carrying implementing the SVM classifier. The function 
$\mathrm{ksvm}$ has an optional parameter called kernel which is set to vanilladot in our implementation. The resultant SVM is a linear one as the training data exhibited linear characteristics.

We now briefly discuss the regression models.

Multivariate Regression: In this regression approach, we use open_perc as the response variable and the remaining ten variables as the predictors to build predictive models for the three cases mentioned earlier in Section 3 . In all these cases, we use the programming language $\mathrm{R}$ for data management, model construction, testing of models, and visualization of results.

Case I: We use 2013 data as the training data set for building the model, and then test the model using the same data set. For both the stocks, we used two approaches of multivariate regression - (i) backward deletion and (ii) forward addition of variables. Both approaches yielded the same results for the stock price data.

For the year 2013, we apply the vif function in the faraway library to detect the collinear variables to get rid of the multicollinearity problem. The variance inflation factor (VIF) values of the variables are found to be as follows: month $=1.003$, day_month $=1.008$, day_week $=1.002$, time $=1.095$, high_perc $=4372$. 547 , low_perc $=4369.694$, close_perc $=165.436$, vol_perc $=1.072$, nifty_perc $=1.046$, range_diff $=$ 156.198. Hence, it is clear that high_perc, low_perc, close_perc, and range_diff exhibit multicollinearity. We retain low perc and range_diff for the model construction and remove the other two variables since their VIF values are smaller than the other two. Using the dropl function in the case of the backward deletion technique and the addl function in the case of the forward addition technique, we identify the variables that are not significant in the model and do not contribute to the information content of the model. For identifying the variables that contribute least to the information contained in the model in each iteration, we use the Akaike Information Criteria (AIC) - the variable that has the least AIC value and non-significant $p$-value in each iteration, is removed from the model in case of the backward deletion process. On the other hand, the variable that has the lowest AIC and a significant $p$-value is added to the model in each iteration for the forward addition technique. It is found that low_perc and range_diff are the two predictors that finally remain in the regression model.

Case II: For the year 2014, the VIF values for the predictors are found to be as follows: month $=1.007$, day_month $=1.004$, day_week $=1.007$, time $=1.057$, high_perc $=1161.446$, low_perc $=1331.035$, close perc $=115.161$, vol_perc $=1.022$, range_diff $=92.092$, nifty_perc $=1.073$. The variables high_perc, low_perc, close_perc, and range_diff exhibit multicollinearity. As in Case I, we retain low_perc and range_diff as their VIF values are smaller compared with the other two. Use of backward deletion and forward addition methods both yield the same regression models as in Case I with low_perc and range_diff as the predictors and open_perc as the response variable.

Case III: In this case, the model is identical to that of Case I. However, the model is tested on the data of the year 2014. Therefore, the performance results of the model are expected to be different. The performance results and their critical analysis are presented in Section 6.

Multivariate Adaptive Regression Spline: Multivariate Adaptive Regression Spline (MARS) is a statistical machine learning approach for building robust regression models. MARS works by splitting input variables into multiple basis functions and then fitting a linear regression model to those basis functions. The basis functions used by MARS are designed in pairs: $f(x)=\{x-t$, if $x>t, 0$ otherwise $\}$ and $g(x)=$ $\{t-x$, if $x<t, 0$ otherwise $\}$. The main characteristic property of the basis functions is that these 
functions are piecewise linear. The value $t$ at which the two functions meet is called a knot. The working principles of MARS are very similar to that of CART. Like CART, MARS first builds a complex model involving a large number of basis functions, which are separated from each other by a large number of knots. This phase of the algorithm execution is called the forward pass of the model building. In the subsequent phase, known as the backward pass, the algorithm prunes back unimportant terms (i.e., basis functions), which could not contribute significantly to the generalized $R^{2}$ values of the model. This phase essentially enables MARS to avoid the possibility of overfitting of the model during the training phase. During the execution of the backward pass, the algorithm computes the generalized cross-validation (GCV) values to determine how well the model fits into the data while avoiding any overfitting. Finally, the algorithm returns the model with the best cost/benefit ratio. To fit a model using MARS in R, we use the function earth in the library earth.

Decision Tree: For building a decision tree regression model, we use the same tree function in the tree library in $\mathrm{R}$ as we did in the decision tree-based classification model. However, in this case, the response variable (i.e., open_perc) is a continuous numeric variable. The predict function is used to predict the values of the response variable. The functions cor and rmse defined in the library Metrics are used to compute the correlation coefficient and the RMSE value for determining the prediction accuracy of the models.

Bagging: We use the bagging function defined in the ipred library of $\mathrm{R}$ for building a bagging regression model. The value of the parameter nbag is set to 100 so that one hundred decision trees are ensembled in the regression model. The predict function in the ipred library is used to predict the response variable values. The rmse function in the Metric library is used to compute the RMSE of the predicted values. The cor function is used to compute the correlation between the actual and the predicted values.

Boosting: We use the blackboost function defined in the mboost library in $\mathrm{R}$ for building boosting regression models on the stock price data. As in other regression models, the predict and rmse functions are used to compute the predicted values and the RMSE values of the regression model.

Random Forest: We use the randomForest function defined in the randomForest library in $\mathrm{R}$ for fitting a random forest regression model into the training data. The response variable open_perc is a numeric variable, and the same predict, rmse, and cor functions are used as in the case of other regression methods.

Artificial Neural Network: As in the case of classification, we use the neuralnet function defined in the neuralnet library in $\mathrm{R}$ for regression on the stock price data. The predictors are normalized using min-max normalization before building the model. The compute function defined in the neuralnet library is used for computing the predicted values, while the parameter hidden is used to change the number of nodes in the hidden layer. The value of the parameter stepmax is set to $10^{6}$ to exploit the maximum number of iterations executed by the neuralnet function. The parameter linear.output is by default set to TRUE, and hence it is not altered. For the Godrej Consumer Products training dataset, only one node in the hidden layer for all the three cases was sufficient for building ANN regression models.

Support Vector Machine: For building the SVM regression model, we use the svm function defined in the e1071 library in R. The predict function is used for predicting the response variable values using the regression model, and the rmse function is used to compute the RMSE values for the predicted quantities.

\section{Deep Learning Models}


In this section, we discuss two deep learning-based regression methods: (i) the long-and short-term memory (LSTM) network, and (ii) the convolutional neural networks (CNNs).

Long- and Short-Term Memory Network: LSTM is a variant of recurrent neural networks (RNNs) - neural networks with feedback loops (Geron, 2019). In such networks, output at the current time slot depends on the current inputs as well as the previous state of the network. However, RNNs suffer from the problem that these networks cannot capture long-term dependencies due to vanishing or exploding gradients during backpropagation in learning the weights of the links (Geron, 2019). LSTM networks overcome such problems, and hence such networks are quite effective in forecasting in multivariate time series. LSTM networks consist of memory cells that can maintain their states over time using memory and gating units that regulate the information flow into and out of the memory. There are different variants of gates used. The forget gates control what information to throw away from memory. The input gates are meant for controlling the new information that is added to the cell state from the current input. The cell state vector aggregates the two components - the old memory from the forget gate, and the new memory from the input gate. In the end, the output gates conditionally decide what to output from the memory cells. The architecture of an LSTM network along with the backpropagation through time (BPTT) algorithm for learning provides such networks a very powerful ability to learn and forecast in a multivariate time series framework. We use Python programming language and the Tensorflow and Keras deep learning frameworks for implementing LSTM networks. While building the LSTM models, we use the open price of the stock as the response variable, and the variables high, low, close, volume and NIFTY, are used as the predictors. Unlike the machine learning techniques, for the LSTM models, we don't compute the differences between successive slots. Rather, we forecast the open value of the next slot based on the values of the response and the predictor variables in the previous slots.

We use the mean absolute error (MAE) as the loss function and the adaptive moment estimation (ADAM) as the optimizer for evaluating the model performance in all three cases. ADAM computes adaptive learning rates for each parameter in the gradient descent algorithm. In addition to storing an exponentially decaying average of the past squared gradients, ADAM also keeps track of the exponentially decaying average of the past gradients, which serves as the momentum in the learning process. Instead of behaving like a ball running down a steep slope like momentum, ADAM manifests itself like a heavy ball with a rough outer surface. This high level of friction results in ADAM's preference for a flat minimum in the error surface. Due to its ability to integrate adaptive learning with a momentum, ADAM is found to perform very efficiently in optimizing the performance of large-scale networks. This was the reason for our choice of ADAM as the optimizer in our LSTM modeling.

We train the LSTM networks using different epoch values and batch sizes for the three different cases. The sequential constructor in the Tensorflow framework is used in building the LSTM model. The performance results of the LSTM models are presented in Section 6.

Convolutional Neural Networks: CNNs emerged from the study of the brain's visual cortex, and they have been used in image recognition since the 1980s. In the last few years, thanks to the increase in computational power, the amount of available training data, and the tricks for training deep neural networks. CNNs have managed to achieve superhuman performance on some complex visual tasks. They power image search services, self-driving cars, automatic video classification systems, and more. Moreover, CNNs are not restricted to visual perception: they are also successful at many other tasks, such as voice recognition, natural language processing, and complex time series analysis of financial data (Binkowski et al., 2017; Lahmiri, 2014). 
In the present work, we exploit the power of $\mathrm{CNN}$ in forecasting the univariate and multivariate time series data of Godrej Consumer Products stock. CNNs have two major types of processing layers - convolutional layers and pooling or subsampling layers. The convolutional layers read an input such as a 2-dimensional image or a one-dimensional signal using a kernel (also referred to as the filter) by reading the data in small segments at a time, and scan across the input data field. Each read result is an interpretation of the input that is projected onto a filter map and represents an interpretation of the input. The pooling or the subsampling layers take the feature map projections and distill them to the most essential elements, such as using a signal averaging (average pool) or signal maximizing process (max pool). The convolution and pooling layers are repeated at depth, providing multiple layers of abstraction of the input signals. The output of the final pooling layer is fed into one or more fully-connected layers that interpret what has been read and maps this internal representation to a class value.

We use the power of CNN in multi-step time series forecasting in the following way. The convolutional layers are used to read sequences of the input data, and automatically extract features. The pooling layers are used for distilling the extracted features, and in focusing attention on the most salient elements. The fully connected layers are deployed to interpret the internal representation and output a vector representing multiple time steps. The benefits that $\mathrm{CNN}$ provides in our time series forecasting job are the automatic feature learning, and the ability of the model to output a multi-step vector directly.

We build three different types of CNN models for multi-step time series forecasting of stock prices. They are: (i) Multi-step time series forecasting with univariate input data, (ii) Multi-step time series forecasting with multivariate input data via channels - in this case, each input sequence is read as a separate channel, like different channels of an image (e.g., red, green, and blue), (iii) multi-step time series forecasting with multivariate input data via sub-models - in this case, each input sequence is read by a different CNN submodel and the internal representations are combined before being interpreted and used to make a prediction.

In the first case, we design a CNN for multi-step time series forecasting using only the univariate sequence of the open values. In other words, given some number of prior days of open values, the model predicts the next standard week of stock market operation. A standard week consists of five days - Monday to Friday. The number of prior days used as the input defines the one-dimensional (1D) data of open values that CNN will read and learn for extracting features.

The multi-step time series forecasting approach is essentially an autoregression process. Whether univariate or multivariate, the prior time series data is used for forecasting the values for the next week.

\section{Performance Results and Analysis}

In this Section, we provide a detailed discussion on the implementation, training, and testing of the predictive models and their performance results. We first discuss the classification techniques and then the regression techniques. The metrics that have been used in evaluating the classification models are as follows:

Sensitivity: It is the ratio of the number of true positives to the total number of positives in the test dataset, expressed as a percentage. Here, positive refers to the cases that belong to the target group (i.e., the class 
"1"). The term true positive refers to the number of positive cases that the model correctly identified. The word sensitivity is also sometimes referred to as recall.

Specificity: It is the ratio of the number of true negatives to the total number of negatives in the test dataset, expressed as a percentage. Here, negative refers to the cases that belong to the non-target group (i.e., the class " 0 "). The term true negative refers to the number of negative cases that the model correctly classified.

Positive Predictive Value: Positive predictive value (PPV), also sometimes referred to as precision, refers to the accuracy of the model in classifying the target group cases among the total number of target group cases identified by it. It is computed as the ratio of the number of correctly classified target group cases to the total number of target group cases as identified by the model. Since the total number of target group cases identified by the model is the sum of the number of true positive cases and the number of falsepositive cases, PPV is the ratio of the total number of true positive cases to the sum of the number of true positive cases and the number of false-positive cases, expressed as a percentage. The complement of PPV is also called the false discovery rate (FDR).

Negative Predictive Value: Negative predictive value (NPV) refers to the accuracy of the model in classifying the non-target group cases among the total number of non-target elements identified by it. NPV is computed as the ratio of the number of correctly identified non-target group cases to the total number of non-target group cases as identified by the model. Since the total number of non-target group cases classified by the model is the sum of the number of true negative cases and the number of false-negative cases, NPV is the ratio of the total number of true negative cases to the sum of the number of true negative cases and the number of false-negative cases, expressed as a percentage. The complement of NPV is also called the false-omission rate (FOR).

Classification Accuracy (CA): It is the ratio of the total number of cases that are correctly classified to the total number of cases in the dataset, expressed as a percentage.

F1 Score: If the test data set is highly imbalanced, with the cases belonging to the non-target group far outnumbering the number of target group cases, sensitivity is usually found to be very poor even when the classification accuracy may be high. Hence, classification accuracy is not considered a very robust and reliable metric. F1 score, which is computed as the harmonic mean of the sensitivity and PPV, is found to be a very robust metric, however.

\section{Classification Methods:}

Logistic Regression: We use glm function in the R programming language to build logistic regressionbased classification models. The response variable (i.e., open_perc) is converted into a categorical type by using the function as.factor before building the models. The parameter family is set to binomial to build a binary logistic regression model. The predict function is used to predict the class of the test data records. We also build the lift curve and the receiver operating characteristic (ROC) curve of the model for each case. The output of the performance function defined in the ROCR library is plotted to depict the ROC curve of the model. The area under the curve (AUC) for each ROC curve is computed using the auc function defined in the $p R O C$ library in the $\mathrm{R}$ programming language.

Table 1: Logistic regression classification results

\begin{tabular}{|l|l|l|l|}
\hline & Case I & Case II & Case III \\
\hline
\end{tabular}




\begin{tabular}{|l|c|c|c|}
\hline \multicolumn{1}{|c|}{ Metrics } & Training Accuracy 2013 & Training Accuracy 2014 & Test Accuracy 2014 \\
\hline Sensitivity & 94.79 & 94.83 & 92.10 \\
\hline Specificity & 97.61 & 95.96 & 89.39 \\
\hline PPV & 96.87 & 95.12 & 87.83 \\
\hline NPV & 96.01 & 95.72 & 93.16 \\
\hline CA & 96.38 & 95.45 & 90.62 \\
\hline F1 Score & 95.82 & 94.97 & 89.91 \\
\hline
\end{tabular}

Table 1 presents the performance results of the logistic regression classification method. For Case I, out of 419 actual "0" cases, only 10 cases are misclassified as " 1 ", while among 326 actual " 1 " cases, 17 cases are found to be wrongly classified as " 0 ". The value of AUC for the ROC curve for Case I is 0.9934 . For Case II, 16 cases out of total 396 actual " 0 " cases are misclassified as " 1 ", and out of 329 cases which are actually "1", are wrongly classified as "0". The AUC value for this case is found to be 0.9891 . Case III yields 42 cases which are actually " 0 " but misclassified as " 1 " out of a total of 396 cases, while among 329 cases which are actually "1", 26 cases are misclassified as "0". The AUC value for Case III is found to be 0.9587 .
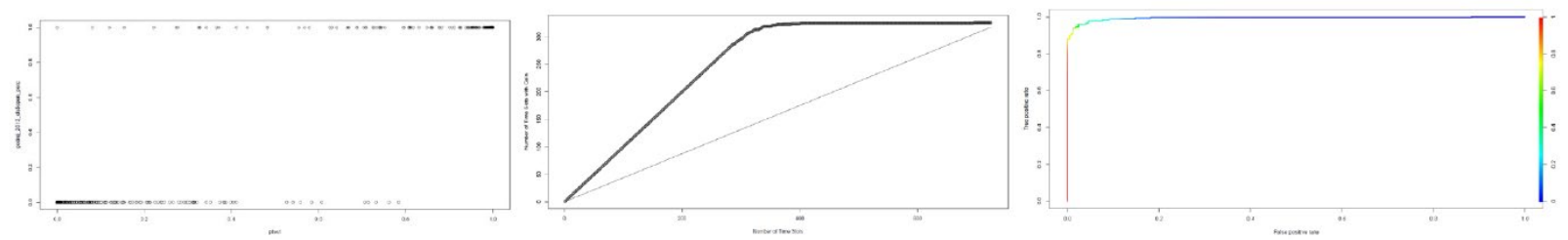

Figure 1: Logistic regression for Case I - (a) actual vs predicted probabilities of open_perc, (b) the lift curve, and (c) the ROC curve

Fig. 1 presents the classification performance, the lift curve, and the ROC curve of the logistic regressionbased classification model for Case I. In Fig. 1(a), the $y$-axis represents the actual classes of the records (either " 0 " or " 1 ") and the $x$-axis denotes the probability that a case will belong to the class " 1 ". The threshold value along the $x$-axis is by convention taken to be 0.5 . Hence, all the cases which are found to be lying on the level " 0 " along the $y$-axis and situated to the right of the threshold value of 0.5 along the $x$ axis are misclassified. Similarly, all the points which are on the level "1" along the $y$-axis, and are situated to the left of the threshold value of 0.5 along the $x$-axis are also misclassified. It is evident from Fig. 1(a) that the number of misclassified cases in Case I is very low. Fig. 1(b) shows that the lift curve is pulled up from the baseline indicating that the model is very effective in discriminating between the two classes. Fig. 1(c) depicts the ROC curve for the logistic regression model for Case I. The steepness of the curve makes it evident that the model can very effectively optimize the values of the true positive rate (TPR) and the false positive rate (FPR). In Fig. 1(c), the line segment with red color presents the class "1" cases that are correctly classified, while the blue line segment denotes the correctly classified cases which belong to the class " 0 ". The portion of the ROC curve that is colored with yellow represents those cases that belong to the class " 0 ", but the model wrongly classified them to the class " 1 ". The green-colored portion of the ROC curve depicts those cases which are misclassified into the class " 0 ", while they belong to the class " 1 ". 

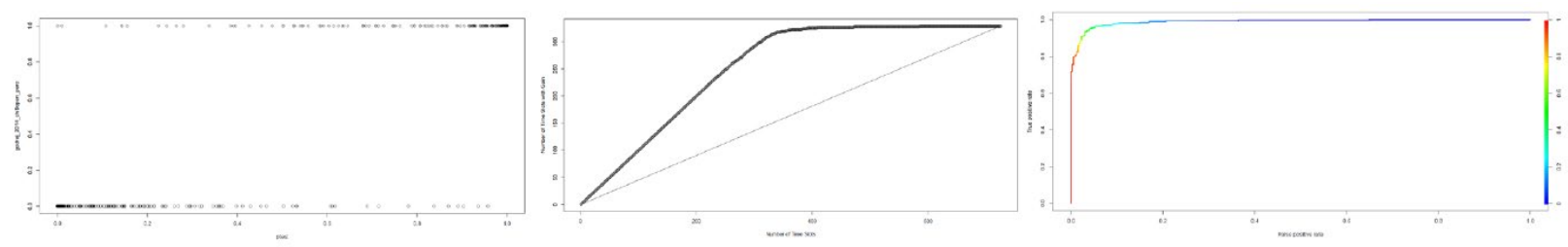

Figure 2: Logistic regression for Case II - (a) actual vs predicted probabilities of open_perc, (b) the lift curve, and (c) the ROC curve

Fig. 2 depicts respectively the classification performance, the lift curve, and the ROC curve of the logistic regression model for Case II. The performance of the model, in this case, is similar to that in Case I. However, the AUC value yielded by the model under this case is just marginally smaller than the corresponding value in Case $I$.
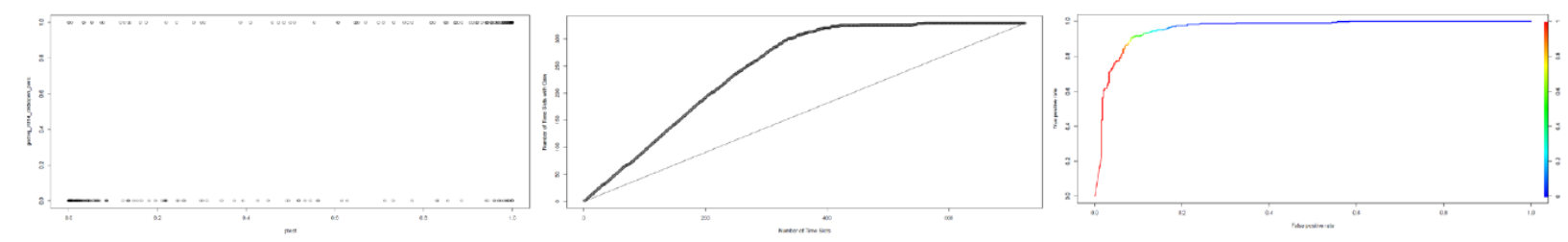

Figure 3: Logistic regression for Case III - (a) actual vs predicted probabilities of open_perc, (b) the lift curve, and (c) the ROC curve

Fig. 3 shows the classification accuracy, the lift curve, and the ROC curve for the logistic regression model in Case III. It is evident from Fig. 3(c) that the classification model in Case III has committed more errors as compared to Case I and Case II. This case also yields a lower AUC value of 0.9587 .

KNN Classification: Table 2 presents the performance results of the KNN classification method. For Case $I$, with the values of $k=1,3,5,7$, and 9, the classification accuracy values are found to be $100,93.42$, $91.68,92.35$, and 92.08 respectively. We choose $k=3$ to avoid the overfitted model with $k=1$. In this case, 419 cases are $0 \mathrm{~s}$ and 326 cases are $1 \mathrm{~s} .15$ cases of actual $0 \mathrm{~s}$ are misclassified as $1 \mathrm{~s}$, and 34 cases of actual $1 \mathrm{~s}$ are misclassified as $0 \mathrm{~s}$. In Case II, for $k=1,3,5,7$, and 9, the classification accuracy values are 100, $90.21,85.10,83.22$, and 84.16 respectively. Again $k=3$ is chosen to avoid model overfitting. 28 cases of actual 0 are misclassified as 1 , while 43 cases of actual 1 are misclassified as 0 . For Case III, the classification accuracy values are found to be $65.24,65.10,67.17,68.69$, and 67.44 for $k=1,3,5,7$, and 9 respectively. We choose $k=3$, for which 202 cases which are 0 s, are misclassified as $1 \mathrm{~s}$, while 51 cases of actual $1 \mathrm{~s}$ were misclassified as $0 \mathrm{~s}$.

Table 2: KNN classification results

\begin{tabular}{|c|c|c|c|}
\hline \multirow{2}{*}{ Metrics } & Case I & Case II & Case III \\
\cline { 2 - 4 } & Training Accuracy 2013 & Training Accuracy 2014 & Test Accuracy 2014 \\
\hline Sensitivity & 89.57 & 86.93 & 84.50 \\
\hline Specificity & 96.42 & 92.93 & 48.99 \\
\hline
\end{tabular}




\begin{tabular}{|l|l|l|l|}
\hline PPV & 95.11 & 91.08 & 57.92 \\
\hline NPV & 92.24 & 89.54 & 79.18 \\
\hline CA & 93.42 & 90.21 & 65.10 \\
\hline F1 Score & 92.26 & 88.96 & 68.73 \\
\hline
\end{tabular}

Decision Tree Classification: We use the tree function defined in the tree library in the R programming language for building the decision tree-based classification models. The response variable open_perc is converted into a categorical type using the as.factor function for classification. The predict function in the tree library is used for predicting the classes of the response variable for the records in the test dataset. For Case I and Case III the models are identical as they are trained on the same training dataset of the year 2013. However, while the model in Case I is tested on the 2103 data, the 2014 data is used for testing the model in Case II. For all three cases, we find that high_perc, low_perc, and close_perc are the three predictor variables that are used in constructing the models. However, in Case I, the predictor which is used for splitting at the root node is close prec, indicating that close perc is the most important predictor for classification in the 2013 dataset. However, for the 2014 dataset, high_perc is found to be the most discriminating one as the same is used by the model for splitting at the root node. In Case I, the decision tree classifier misclassifies 8 cases out of a total of 419 cases which belong to the class " 0 ", while 16 cases are misclassified out of a total of 326 cases which are the records of the class " 1 ".

Table 3: Decision Tree classification results

\begin{tabular}{|l|c|c|c|}
\hline \multirow{2}{*}{ Metrics } & Case I & Case II & Case III \\
\cline { 2 - 4 } & Training Accuracy 2013 & Training Accuracy 2014 & Test Accuracy 2014 \\
\hline Sensitivity & 95.09 & 92.40 & 89.97 \\
\hline Specificity & 98.09 & 95.71 & 92.42 \\
\hline PPV & 97.48 & 94.70 & 90.80 \\
\hline NPV & 96.25 & 93.81 & 91.73 \\
\hline CA & 96.78 & 94.21 & 91.31 \\
\hline F1 Score & 96.27 & 93.54 & 90.38 \\
\hline
\end{tabular}

In Case II, the model fails to classify correctly 17 cases out of a total of 396 cases which are actually " 0 " class members, while 25 cases are misclassified out of a total of 329 cases that belong to the class " 1 ". Case $I I I$, faces a more challenging task. We find that the model is unable to correctly classify 30 cases out of a total of 396 cases that belong to the class " 0 ", while 33 cases are misclassified out of a total of 329 cases that belong to the actual class of " 1 ". Table 3 presents the performance results of the decision tree classification models under three different cases. Fig. 4 depicts the decision tree classifiers for Case I, Case $I I$, and Case III respectively. 


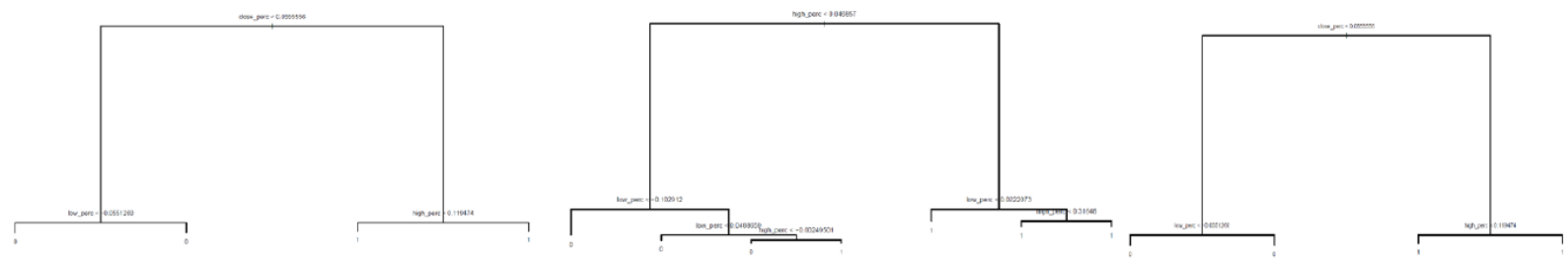

Figure 4: Decision tree for classification - (a) tree for Case I, (b) the tree for Case II, and (c) the tree for Case III

Table 4: Bagging classification results

\begin{tabular}{|l|c|c|c|}
\hline \multirow{2}{*}{ Metrics } & Case I & Case II & Case III \\
\cline { 2 - 4 } & Training Accuracy 2013 & Training Accuracy 2014 & Test Accuracy 2014 \\
\hline Sensitivity & 95.09 & 95.44 & 89.97 \\
\hline Specificity & 98.09 & 96.46 & 92.42 \\
\hline PPV & 97.48 & 95.73 & 90.78 \\
\hline NPV & 96.25 & 96.22 & 91.73 \\
\hline CA & 96.78 & 96.00 & 91.31 \\
\hline F1 Score & 96.07 & 95.58 & 90.37 \\
\hline
\end{tabular}
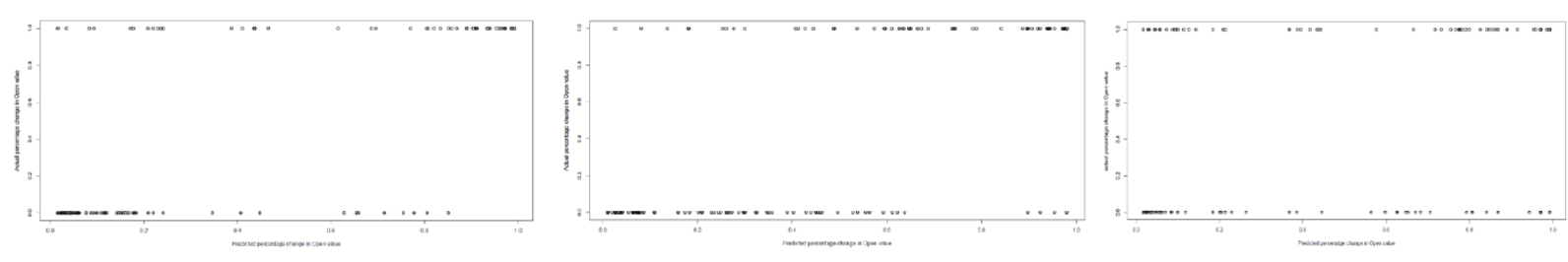

Figure 5: Bagging classification: actual vs predicted open_perc - (a) for Case I, (b) for Case II, and (c) for Case III

Bagging Classification: We used the bagging function defined in the ipred library in the R programming language for building the bagging classification models. We set the value of the parameter $n b a g$ to 25 so that 25 decision trees were created randomly, and a simple majority voting mechanism is applied in constructing the classifier. In Case I, we find that the model fails to correctly classify 8 cases out of a total of 419 cases that belong to the class " 0 ", while 16 cases out of a total of 326 cases that belong to the class "1" are also misclassified. In Case II, the model is unable to correctly classify 14 cases out of a total of 396 cases that are of actual class " 0 ", while 15 cases out of a total of 329 cases are misclassified which belong to the class "1". In Case III, 30 cases out of 396 actual "0" cases are misclassified by the model, while 33 cases out of a total of 329 cases of the class " 1 " are also misclassified. The performance results of the bagging classification model for all three cases are presented in Table 4. Fig. 5 depicts the classification accuracy of the model for Case I, Case II, and Case III respectively. In all these three figures, the $y$-axis represents the actual class labels, while the values along the $x$-axis show the probabilities of the predicted class for the records. The cases which are on the label " 0 " on the $y$-axis and have their probability values greater than 0.5 along the $x$-axis are the misclassified cases. In a similar line, those cases which are lying 
on the label " 1 " along the $y$-axis and have their probability values less than 0.5 along the $x$-axis, are also misclassified.

Table 5: Boosting classification results

\begin{tabular}{|l|c|c|c|}
\hline \multirow{2}{*}{ Metrics } & Case I & Case II & Case III \\
\cline { 2 - 4 } & Training Accuracy 2013 & Training Accuracy 2014 & Test Accuracy 2014 \\
\hline Sensitivity & 100 & 100 & 92.10 \\
\hline Specificity & 100 & 100 & 93.43 \\
\hline PPV & 100 & 100 & 92.10 \\
\hline NPV & 100 & 100 & 93.43 \\
\hline CA & 100 & 100 & 92.83 \\
\hline F1 Score & 100 & 100 & 92.10 \\
\hline
\end{tabular}
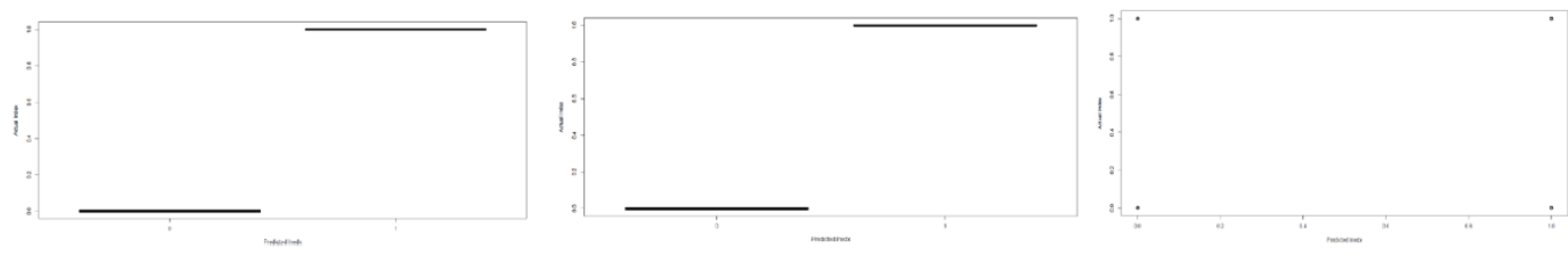

Figure 6: Boosting classification: actual vs predicted open_perc - (a) for Case I, (b) for Case II, and (c) for Case III

Boosting Classification: We use the boosting function defined in the adabag library in the R programming language for building the boosting models for classification. The response variable open_perc is transformed into the categorical type using as.factor function to satisfy the requirement of a classification model. The predict function is used for predicting the class of the response variable in the test data records. For both Case I and Case II, the boosting classification models are found to be yielding $100 \%$ accuracy in all the metrics of classification. This may be observed in Table 5. This is not surprising as in both the cases the models were built and tested using the same dataset, and thus the learning of the models had been very accurate using the ensemble of the weighted majority voting on a large number of random decision tree classifiers. However, the model encounters a more difficult task in Case III, in which the ensemble model is built on the 2013 data, and then tested on the 2014 data. In Case III, we find that the model misclassifies 26 cases out of a total of 396 cases that belong to the class " 0 ", while among 329 cases that are actually of the class " 1 ", 26 cases are incorrectly classified. Table 5 presents the performance results of the boosting classification models. Fig. 6 depicts the performance of the boosting classifier for Case I, Case II, and Case III respectively. In these three figures, along the $y$-axis the actual classes are plotted - there are two actual class levels " 0 " and " 1 ". The $x$-axis presents the predicted probability that a case will belong to the class "1". Hence, the data points which are situated to the left side of the threshold value of 0.5 along the $x$-axis, and lying on the level "1" along the $y$-axis are the misclassified cases. Similarly, the points that are on the 
right side of the threshold value of 0.5 , and lying on the level " 0 " along the $y$-axis are also the misclassified cases. It is evident from Fig. 6 that boosting classifiers have performed very well in all three cases.

Table 6: Random Forest classification results

\begin{tabular}{|l|c|c|c|}
\hline \multirow{2}{*}{ Metrics } & Case I & Case II & Case III \\
\cline { 2 - 4 } & Training Accuracy 2013 & Training Accuracy 2014 & Test Accuracy 2014 \\
\hline Sensitivity & 94.48 & 93.01 & 91.19 \\
\hline Specificity & 97.61 & 94.19 & 92.93 \\
\hline PPV & 96.86 & 93.01 & 91.46 \\
\hline NPV & 98.08 & 94.19 & 92.70 \\
\hline CA & 96.24 & 93.66 & 92.14 \\
\hline F1 Score & 95.66 & 93.01 & 91.32 \\
\hline
\end{tabular}

Random Forest Classification: We use the randomForest function defined in the randomForest library in $\mathrm{R}$ programming language, for building random forest-based classification models. In all three cases, the random forest algorithm creates 500 decision trees using three predictors at each node in the decision trees for carrying out the splitting task. In Case I, the model misclassifies 10 cases as the class "1" cases out of a total of 419 cases that belong to the class " 0 ". On the other hand, 18 cases are misclassified into the class " 1 " out of a total of 326 cases which are actually of the class " 0 ". The out-of-bag (OOB) estimate of the error rate of the model, in this case, is $3.76 \%$. In Case II, the model fails to classify correctly 23 cases out of a total of 396 cases that belong to the actual class of " 0 ". On the other hand, 23 cases out of a total of 329 cases that belong to the class of " 0 " are also misclassified. The OOB estimate of the error rate of the classification model, in this case, is $6.34 \%$. In Case III, the random forest classification model was identical to that in Case I. However, the model was tested on 2014 data unlike the model in Case I that was tested on 2013 data. We find that in Case III, the model misclassifies 28 cases out of a total number of 396 cases that belong to the class " 0 ". On the other hand, 29 cases are wrongly classified out of a total of 329 actual "1" cases. The performance results of the random forest classification models are presented in Table 6 .

ANN Classification: We use the neuralnet function defined in the neuralnet library in the R programming language to build ANN classification models. The parameter linear.output is set to false, and the response variable open_perc is converted into a categorical variable type by using the function as.factor before the classification models are built. We find that only one node at the hidden layer is sufficient to model the data. Hence, we pass the value of the parameter hidden as 1 in the neuralnet function. To avoid any possible scenario in which the backpropagation algorithm fails to converge, we set the parameter stepmax to its maximum possible value of $10^{6}$. In Case I, the ANN classification model misclassifies 10 cases out of a total of 419 cases as " 1 ", while they belong to the class " 0 ". On the other hand, 15 cases which are actually " 1 ", are misclassified as " 0 " out of a total of 326 cases. The ANN model for classification for Case I and its performance in the classification task is presented in Fig. 7. In Fig. 7(b), the actual class labels are plotted along the $y$-axis, and along the $x$-axis, the predicted probabilities are plotted. The points lying on the actual class label " 0 " along the $y$-axis while having their predicted class probabilities greater than 0.5 (i.e., those points on the " 0 " label lying on the right-hand side of the threshold value of 0.5 along the $x$-axis) represent 
the misclassified cases. In a similar line, the points which are on the label "1" along the $y$-axis while having their probabilities smaller than 0.5 (i.e., those points on the "1" label lying on the left-hand side of the threshold value of 0.5 along the x-axis) are also misclassified points. In Case II, the ANN classification model misclassifies 17 cases as class " 0 " out of 396 cases that belong to the class " 1 ". On the other hand, 21 cases are misclassified as class " 1 " out of 329 cases which are class " 0 " cases.
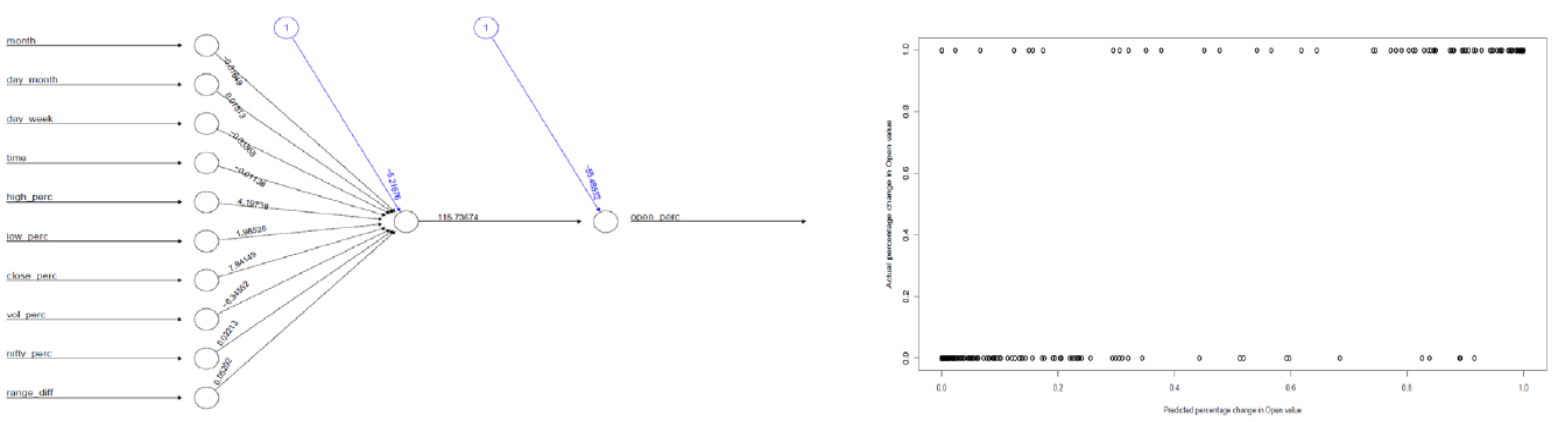

Figure 7: ANN classification - (a) the model for Case I, (b) actual vs predicted open_perc for Case I
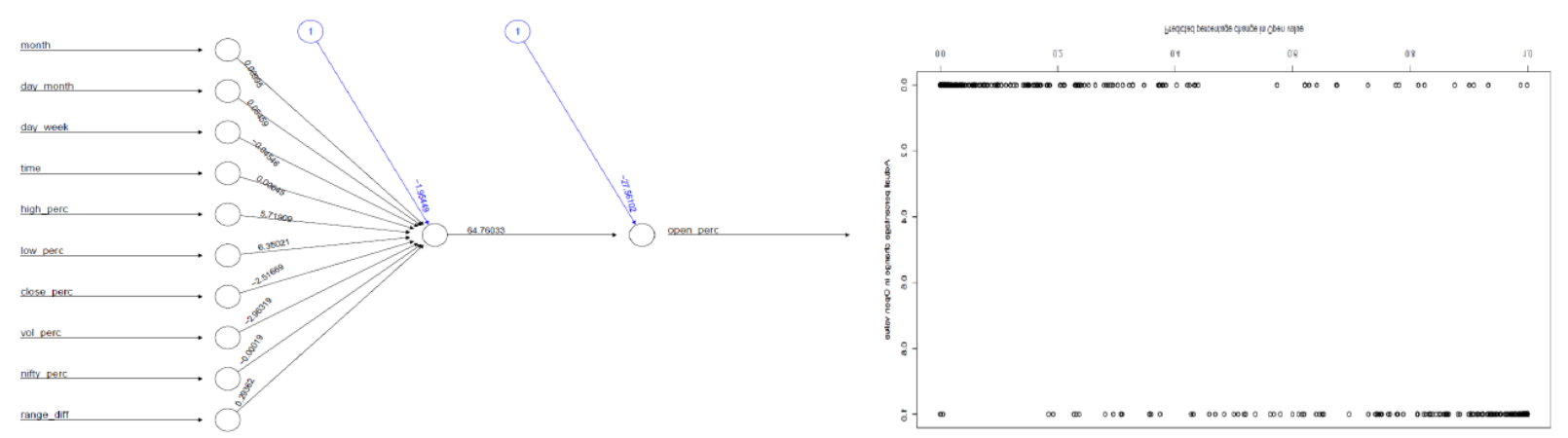

Figure 8: ANN classification - (a) the model for Case II, (b) actual vs predicted open_perc for Case II

Fig. 8 presents the ANN classification model in Case II, and its performance in the classification task, respectively. In Case III, the model is built using 2013 data, hence it is identical to the model that is used in Case I. However, since the model is tested on 2014 data, unlike in Case I in which the model is tested on 2013 data, the performance results of the model in Case III are quite different. The model in Case III encounters a more difficult challenge as there are differences in the characteristics of the data in 2013 and 2014. We find that in Case III, the model misclassifies 259 cases as class " 1 " out of 396 cases that belong to the class " 0 ". On the other hand, only 1 case out of 329 cases which are actually of the class " 1 " are misclassified as the class " 0 ". It is evident, that model performs poorly in classifying the class " 0 " cases which results in a very low value of its specificity. The specificity in Case III is found to be only $34.60 \%$, while for Case I and Case II, the specificity values are $97.61 \%$ and $95.71 \%$ respectively. This indicates that the ANN classification model has a poor generalization in its learning during the training phase using the 2013 data, and that possibly led to a model overfitting. The overfitted model fails to correctly classify the majority of the " 0 " cases in the test data of 2014. This results in a very low specificity value. Fig. 9 presents 
the ANN classification model, and its classification performance respectively. Table 7 presents the performance of the ANN classification models.
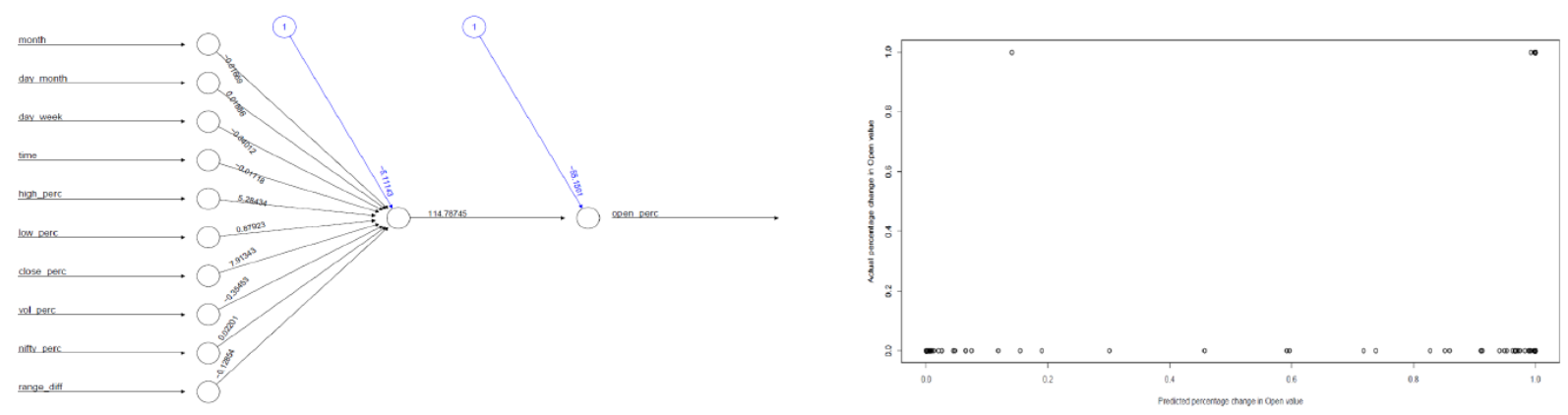

Figure 9: ANN classification - (a) the model for Case III, (b) actual vs predicted open_perc for Case III

Table 7: ANN classification results

\begin{tabular}{|l|c|c|c|}
\hline \multirow{2}{*}{ Metrics } & Case I & Case II & Case III \\
\cline { 2 - 4 } & Training Accuracy 2013 & Training Accuracy 2014 & Test Accuracy 2014 \\
\hline Sensitivity & 95.40 & 93.62 & 99.70 \\
\hline Specificity & 97.61 & 95.71 & 34.60 \\
\hline PPV & 96.88 & 94.77 & 55.88 \\
\hline NPV & 96.46 & 94.75 & 99.28 \\
\hline CA & 96.64 & 94.76 & 64.14 \\
\hline F1 Score & 96.13 & 94.19 & 71.62 \\
\hline
\end{tabular}

SVM Classification: We use the ksvm function defined in the kernlab library in the R programming language for building the SVM-based classification models. The function $\mathrm{ksvm}$ is used with the parameter kernel set to vaniladot. It implies that a linear kernel is used for building the SVM classification models. For Case I, the model finds 120 support vectors. We observe that out of a total number of 430 cases which are actually " 0 " class records, 19 cases are misclassified as " 1 ". On the other hand, 8 cases are misclassified as " 0 ", out of a total of 315 cases which are actually " 1 ". The training error in Case I is found to be $3.62 \%$. For Case II, the model detects 156 support vectors for classifying 725 records. Among 406 cases that belong to the class " 0 ", 27 cases are misclassified as " 1 ". On the other hand, 17 cases are misclassified as "0" out of a total of 319 cases which are actually "1". The training error for Case II is found to be $6.07 \%$. The SVM classification model identifies 116 support vector points in Case III. The model misclassified 41 cases as " 1 " out of a total of 418 cases which are actually " 0 ". On the other hand, out of a total of 307 cases which are actually "1", 19 cases are misclassified as " 0 ". Table 8 presents the results of the SVM classification models.

Table 8: SVM classification results 


\begin{tabular}{|l|c|c|c|}
\hline \multirow{2}{*}{ Metrics } & Case I & Case II & Case III \\
\cline { 2 - 4 } & TrainingAccuracy 2013 & Training Accuracy 2014 & Test Accuracy 2014 \\
\hline Sensitivity & 94.46 & 94.67 & 93.81 \\
\hline Specificity & 95.58 & 93.35 & 90.19 \\
\hline PPV & 94.17 & 91.79 & 87.54 \\
\hline NPV & 98.09 & 95.71 & 95.20 \\
\hline CA & 96.38 & 93.93 & 91.72 \\
\hline F1 Score & 94.31 & 93.21 & 90.57 \\
\hline
\end{tabular}

\section{Regression Methods:}

Multivariate Regression: We already mentioned in Section 44 that the predictors that are finally included in the multivariate regression models in all three cases are: low_perc and range_diff. For Case I, the regression model yields a value of 0.9919 for the adjusted $R^{2}$ value, and the $F$ statistic value of $4.58^{*} 10^{4}$ with an associated $p$-value of $2.2^{*} 10^{-16}$. This indicates that the regression model is successfully able to establish a linear relationship between the response variable, open perc, and the predictor variables, low_perc, and range_diff. The RMSE value yielded by the regression model for this case is found to be 0.0853 , and the mean of the absolute values of the actual open_perc is 0.6402 . The ratio of the RMSE to the mean of the absolute values of the actual open_perc is found to be 13.317. 14 cases out of a total of 745 cases exhibit sign mismatch between the predicted and the actual values of open perc. The correlation test produces the value of the correlation coefficient value as 0.99 with a $p$-value of the $t$-statistic as $2.2 * 10^{-16}$. This indicates that there is a strong linear relationship between the predicted and the actual values of open_perc. The Breusch-Pagan test for homoscedasticity on the residuals yields a test statistic value of 10.239 with a $p$-value of 0.005978 . Hence, it is evident that the residuals are not homoscedastic. However, the Durbin-Watson test of autocorrelation produces a test statistic value of 3.023 with an associated $p$-value of 1 . Hence, the null hypothesis that assumes the presence of no autocorrelation among the residuals has the fullest support. Hence, we conclude that the residuals do not exhibit any significant autocorrelation. For Case II, the regression model yields an adjusted $R^{2}$ value of 0.9827 with the value of the $F$-statistics as $2.052 * 10^{4}$. The $p$-value of the $F$ statistics is found to be smaller than $2.2 * 10^{-16}$ indicating a very highly significant $F$ statistic, and a very good model fit. The RMSE value for Case II is found to be 0.1749 , with the mean of the absolute values of the actual open_perc as 0.9286 . The ratio of the RMSE to the mean of the absolute values of the actual open_perc is found to be 18.84 . 39 cases out of a total of 725 cases have a sign mismatch between the predicted and the actual open_perc values. The correlation test for this case yields a value of correlation coefficient as 0.99 with a value of the $t$-statistic as 202.74 . The $p$-value of the $t$-statistic is $2.2^{*} 10^{-16}$, which indicates a very strong linear relationship between the predicted and the actual open_perc values. The Breusch-Pagan test yields a test statistic value of 3.1877 with an associated $p$-value of 0.203 . Hence, it is evident that the residuals do not exhibit the presence of any significant heteroscedasticity. The Durbin-Watson test of autocorrelation produces a test statistic value of 2.9005 . The $p$-value of the Durbin-Watson test is found to be 1 . This indicates that the null hypothesis of no significant autocorrelation among the residues has received full support. Hence, we conclude that the residuals in the regression model for Case II do not exhibit any significant autocorrelation. The model built under Case III is the same as the one built under Case I. However, its performance results are quite different as it is tested 
on 2014 data, unlike the model in Case I which is tested on 2013 data. The RMSE of the model for Case $I I I$ is found to be 0.1753 , with the mean of the absolute values of the actual open_perc as 0.9286 . Thus, the ratio of the RMSE to the mean of the absolute values of the actual open_perc values is found to be 18.88 . We find that 39 cases out of a total of 725 cases exhibit a sign mismatch between the predicted and the actual values of open_perc. The correlation test yields a correlation coefficient of 0.99 , with the value of the $t$-statistics as 202.53, and the associated $p$-value of $2.2 * 10^{-16}$. This indicates that the predicted and the actual values of open_perc exhibit a strong linear relationship between them. The Breusch-Pagan test yields a test statistic value of 3.1877 with an associated $p$-value of 0.2031 . Hence, it is clear that the residuals are not heteroscedastic. The test statistic value yielded by the Durbin-Watson test is found to be 2.9005 with an associated $p$-value of 1 . Thus, it is evident that the null hypothesis of no autocorrelation among the residuals has gained the fullest support. We conclude that the residuals do not exhibit any significant autocorrelation.

Table 9: Multivariate Regression results

\begin{tabular}{|l|c|c|c|}
\hline \multirow{2}{*}{ Metrics } & Case I & Case II & Case III \\
\cline { 2 - 4 } & Training 2013 & Training 2014 & Test 2014 \\
\hline Correlation Coefficient & 0.99 & 0.99 & 0.99 \\
\hline RMSE/Mean of Absolute Values of Actuals & 13.32 & 18.84 & 18.88 \\
\hline Percentage of Mismatched Cases & 18.67 & 5.38 & 5.24 \\
\hline
\end{tabular}
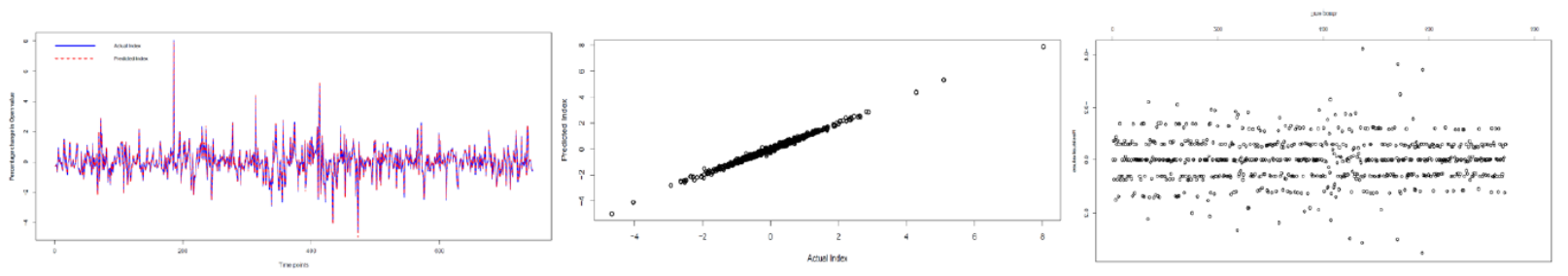

Figure 10: Multivariate regression - (a) actual and predicted values of open_perc for Case I, (b) relationship between actual and predicted open_perc for Case I, (c) residuals for Case I

Table 9 presents the performance results of the multivariate regression models. Fig. 10 presents the performance results of the model under Case I. It is evident from Fig. 10(a) that the predicted values very closely follow the pattern of the variation of the actual open_perc values. Fig. 10(b) exhibits a very strong linear relationship between the predicted and the actual values of open_perc. Fig. 10(c) shows that the residuals of the model are scattered and random, and they do not exhibit any significant autocorrelation. 

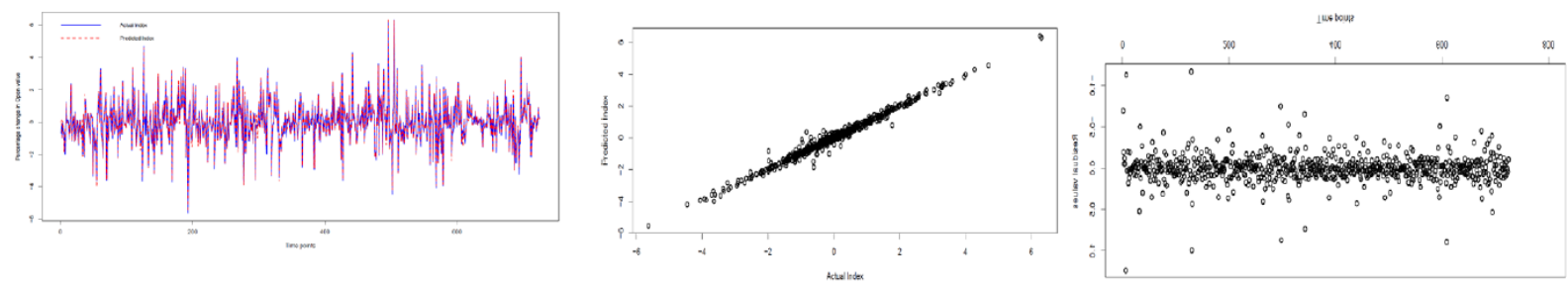

Figure 11: Multivariate regression - (a) actual and predicted values of open_perc for Case II, (b) relationship between actual and predicted open_perc for Case II, (c) residuals for Case II

The performance results of Case II are presented in Fig. 11. The predicted and the actual values of the open_perc exhibit almost identical movement patterns in Case II as in Case I. The residuals do not show any significant autocorrelations. The performance results of the model for Case III are presented in Fig. 12. Fig. 12(a) shows how closely the predicted values of the open_perc follow the patterns exhibited its actual values in Case III, while Fig 12(b) exhibits a strong linear relationship between them. Fig. 12(c) shows that the residuals in Case III are random, and do not exhibit any significant autocorrelations.
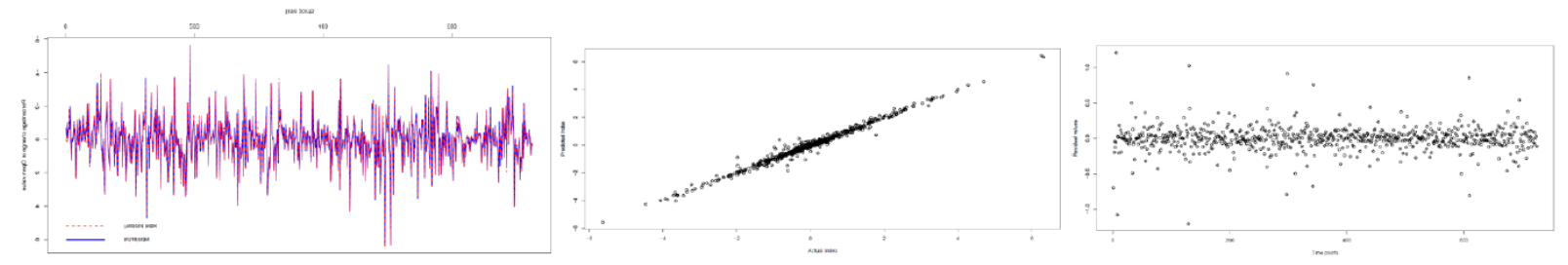

Figure 12: Multivariate regression - (a) actual and predicted values of open_perc for Case III, (b) the relationship between actual and predicted open_perc for Case III, (c) residuals for Case III

Table 10: MARS regression results

\begin{tabular}{|l|c|c|c|}
\hline \multirow{2}{*}{ Metrics } & Case I & Case II & Case III \\
\cline { 2 - 4 } & Training 2013 & Training 2014 & Test 2014 \\
\hline Correlation Coefficient & 0.99 & 0.99 & 0.99 \\
\hline RMSE/Mean of Absolute Values of Actuals & 12.41 & 17.09 & 20.40 \\
\hline Percentage of Mismatched Cases & 1.21 & 4.28 & 6.34 \\
\hline
\end{tabular}

Multivariate Adaptive Regression Spline (MARS): We use the earth function defined in the earth library in the R programming language for building the MARS regression models. For Case I, in the forward pass of the execution of the algorithm, seven terms are used in the model building. After the inclusion of the eighth term. the change in the value of $R^{2}$ is found to be only $5^{*} 10^{-5}$, which is less than the threshold value of 0.001. After the completion of the forward pass, both the generalized $R^{2}$ (GRSq) and the $R^{2}$ converged to a common value of 0.993 . During the backward pass, the algorithm is not able to prune any term, and all the seven terms used in the forward pass are finally retained in the model. In Case 1, the model retains three predictors out of a total of ten predictors. The selected predictors in decreasing order of their importance in the model are found to be: close_perc, high_perc, and low_perc. On the other hand, the predictors which are excluded from the model are: month, day_month, day_week, time, vol_perc, nifty_perc, 
and range_diff. On completion of the execution of the algorithm, the values of some of the important metrics of the model are found to be as follows: (i) generalized cross-validation (GCV): 0.0065 , residual sum of square (RSS): 4.7006, GRSq: 0.9928, and $R^{2}: 0.9930$. The seven terms that are used by the MARS model under Case I are found to be as follows: (i) the intercept, (ii) $h(-0.83682$ - high perc), (iii) $h$ (high_perc $0.83682)$, (iv) $h(-0.692841$ - low_perc), (v) $h($ low_perc - 0.692841), (vi) $h(-2.11268$ - close perc), and (vii) $h$ (close perc - 2.11268). For Case I, the MARS regression model yields 9 cases out of a total of 745 cases that exhibit a mismatch in sign between the predicted and the actual values of open perc. The RMSE value for this case is 0.0794 , while the mean of the absolute values of the actual open_perc is 0.6402 . Hence, the ratio of the RMSE to the mean of the absolute values of the actual open_perc is 2.4065 . The correlation test yields a value of the correlation coefficient as 0.99 with the $t$-statistic value of 325.41 , and an associated $p$-value of $2.2 * 10^{-16}$. This indicates that there is a strong linear relationship between the predicted and the actual values of open_perc. Table 10 presents the results of the MARS regression model.

Fig. 13 presents results of MARS regression under Case $I$.
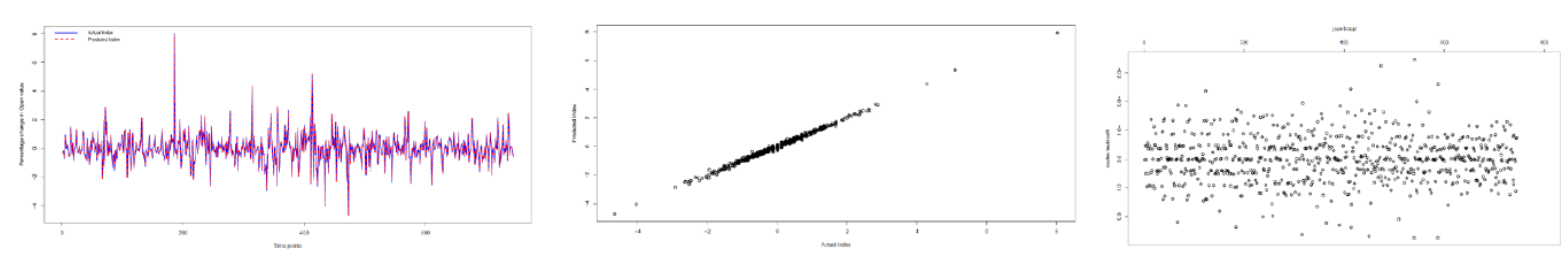

Figure 13: MARS - (a) actual and predicted values of open_perc for Case I, (b) relationship between actual and predicted open_perc for Case I, (c) residuals for Case I
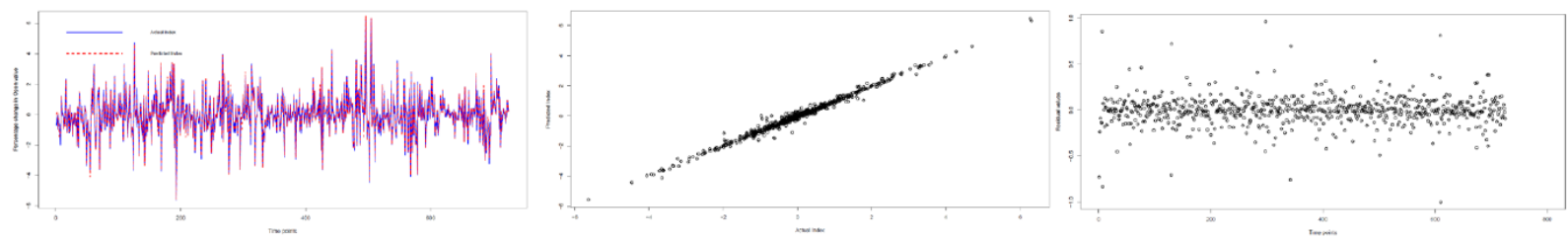

Figure 14: MARS - (a) actual and predicted values of open_perc for Case II, (b) the relationship between actual and predicted open_perc for Case II, (c) residuals for Case II

In Case II, the algorithm used nine terms during its forward execution. The change in the $R^{2}$ value at the end of the ninth term is found to be only 0.0002 , which is less than the threshold value of 0.001 . On completion of the forward pass, the values of GRSq and $R^{2}$ are found to be 0.985 and 0.986 respectively. During the backward pass of its execution, the algorithm prunes one term out of the nine terms that were included earlier during the forward pass. Hence, the algorithm, finally, uses eight terms in building the regression model. We also observe that the algorithm retains four predictors out of a total of ten predictors that were available initially. The four predictors that are retained in the model, in the decreasing order of their importance, are: low_perc, close_perc, range_diff, and high_perc. After the model completes its execution of the backward pass the values of some important metrics are noted. They are as follows: GCV: 0.0262, RSS: 18.2512, GRSq: 0.9852, and $R^{2}$ : 0.9858. In Case II, the algorithm uses the following eight terms: (i) the intercept, (ii) $h\left(0.3675\right.$ - high_perc), (iii) $h\left(h i g h \_p e r c-0.3675\right)$, (iv) $h\left(-2.6685-l o w \_p e r c\right)$, 
(v) $h$ (low_perc - 2.6685), (vi) $h(0.3996$ - close_perc), (vii) $h(-1.8$ - range_diff), and (viii) $h$ (range_diff - 1.8). In Case II, we find that 31 cases out of a total of 725 cases exhibit mismatched signs between the predicted and the actual values of open_perc. With an RMSE value of 0.1587 , and the mean of the absolute values of the actual open_perc as 0.9286 , their ratio is found to be 17.09 . The correlation test yields the value of the correlation coefficient as 0.99 , the $t$-statistic as 223.87 , an associated $p$-value of $2.2 * 10^{-16}$. The high value of the correlation coefficient, and the negligible support for the null hypothesis in the form of a very low $p$-value, indicates that there is a very strong linear relationship between the predicted and the actual values of open_perc. Fig. 14 presents results of MARS regression under Case II.

The MARS model in Case III is identical to that of Case I. The model is, however, tested on the 2014 data. We observe that in Case III, the model yields 46 cases out of a total of 725 cases that exhibit a sign mismatch between the predicted and the actual open perc values. The RMSE of the model, in this case, is found to be 0.1894 , while the mean of the absolute values of the actual open_perc is 0.9286 . The ratio of the RMSE to the mean value is found to be 20.40. The correlation test on the predicted and the actual values of open_perc yields a correlation coefficient value of 0.99 , the value of $t$-statistic as 187.13 , and an associated $p$-value of $2.2 * 10^{-16}$. The results indicate that the predicted and the actual values of open_perc exhibit a strong linear relationship between them for Case III as well. The performance results of the MARS model are presented in Fig. 15.
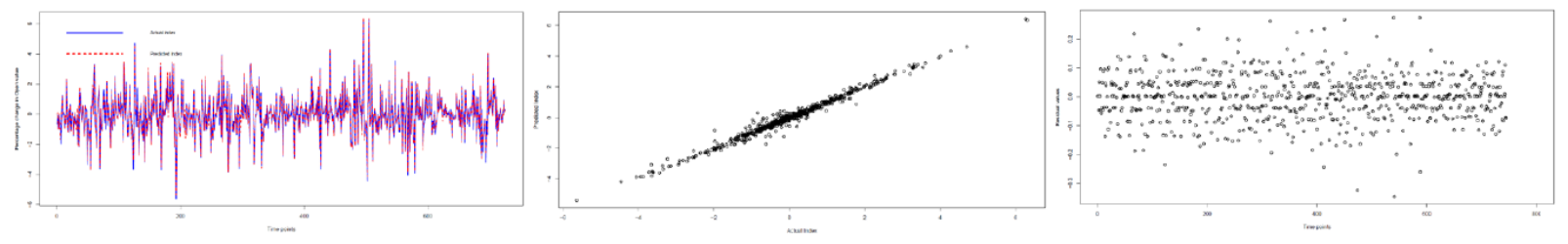

Figure 15: MARS - (a) actual and predicted values of open_perc for Case III, (b) the relationship between actual and predicted open_perc for Case III, (c) residuals for Case III

Table 11: Decision Tree regression results

\begin{tabular}{|l|c|c|c|}
\hline \multirow{2}{*}{ Metrics } & Case I & Case II & Case III \\
\cline { 2 - 4 } & Training 2013 & Training 2014 & Test 2014 \\
\hline Correlation Coefficient & 0.97 & 0.97 & 0.10 \\
\hline RMSE/Mean of Absolute Values of Actuals & 35.35 & 37.04 & 165.92 \\
\hline Percentage of Mismatched Cases & 13.42 & 17.38 & 47.72 \\
\hline
\end{tabular}

Decision Tree Regression: We use the tree function defined in the tree library in the R programming language to build decision tree-based regression models. For Case I, close perc turns out to be the splitting variable at the root node. Other important variables that lead to splitting at nodes are: high perc and low_perc. Fig. 16 depicts the decision tree model under Case I, and the patterns exhibited by the actual and the predicted values of the open_perc for the model. It is evident from Fig 16(b) that barring some minor deviations, the predicted values of open_perc very closely follow the pattern of the actual values. RMSE for this case is 0.2263 , and the mean of the absolute values of the actual open_perc is 0.6402 . Among the total of 745 cases, 100 cases exhibit a sign mismatch between the predicted and the actual values of 
open_perc. The correlation coefficient between the predicted and the actual open_perc turns out to be 0.97. The $t$-statistics for the correlation test yields a value of 111.35 with a $p$-value of $2.2 * 10^{-16}$, indicating a strong linear relationship between the predicted and the actual open_perc values.

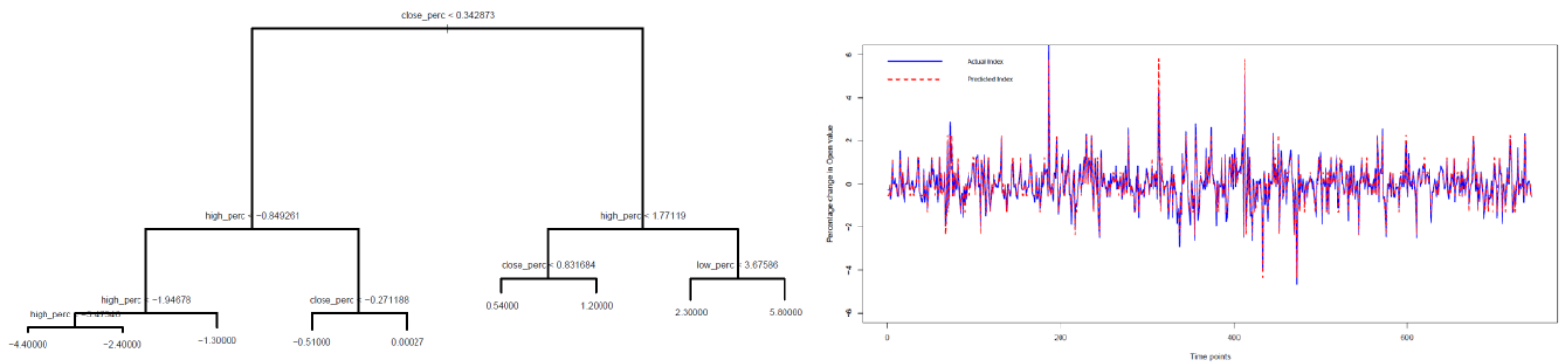

Figure 16: Decision tree regression - (a) the tree model for Case I, (b) actual and predicted open_perc for Case I
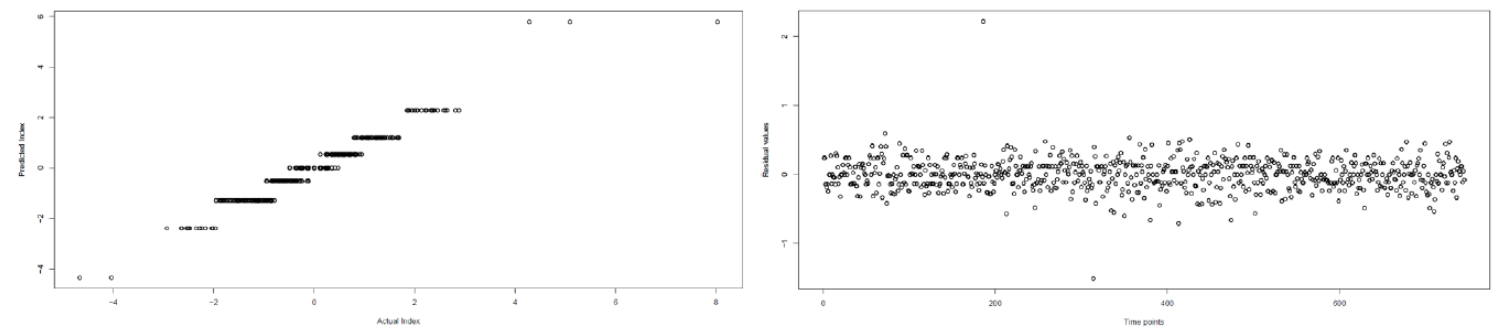

Figure 17: Decision tree regression - (a) the relationship between actual and predicted open_perc values for Case I, (b) residuals of regression for Case I

Fig. 17 depict some additional performance results of the decision tree-based regression model for Case I. Fig 17(a) shows that with the increase in the actual open perc values, its predicted values also exhibit an upward trend, stepwise. Fig 17(b) shows that residuals do not exhibit any significant autocorrelation. Table 11 depicts the results of the decision tree regression model.
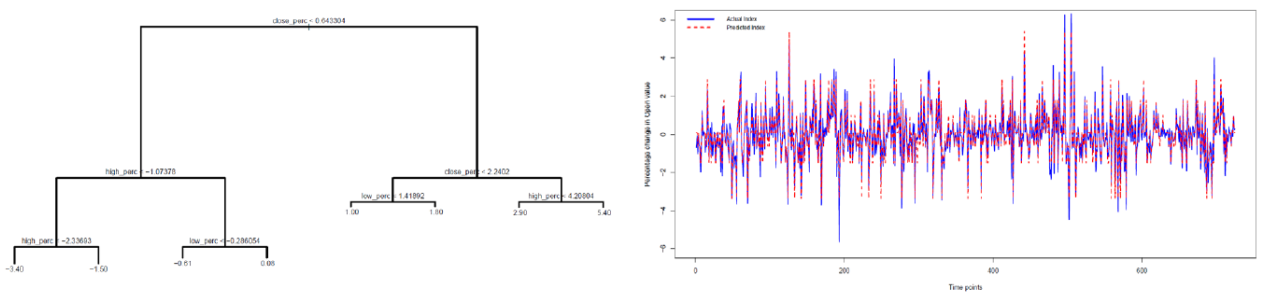

Figure 18: Decision tree regression - (a) the tree model for Case II, (b) actual and predicted open_perc for Case II
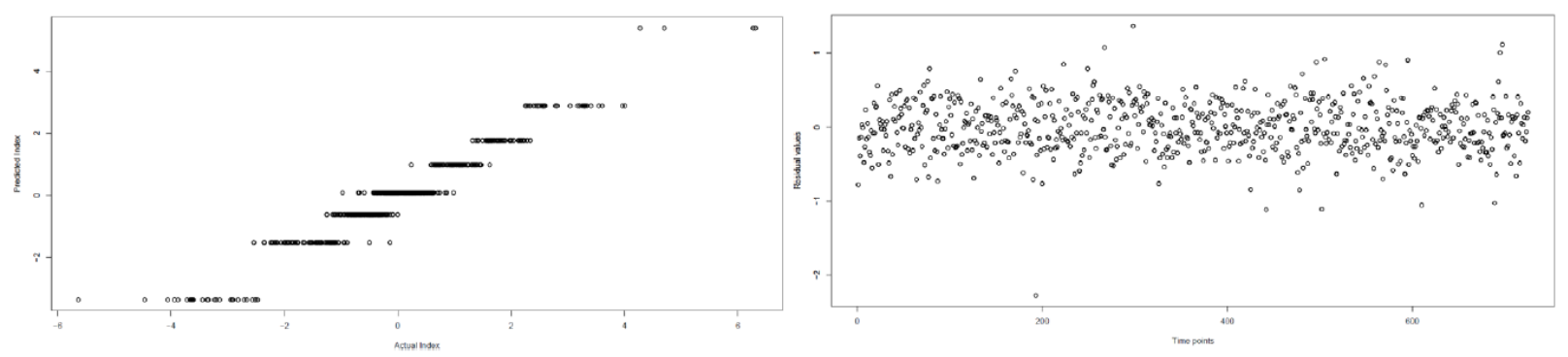
Figure 19: Decision tree regression - (a) the relationship between actual and predicted open perc values for Case II, (b) residuals of regression for Case II

Fig. 18 presents the decision tree regression model for Case II, and the pattern of variations of the actual and the predicted values of open_perc for this model. The predicted values of open_perc are found to closely follow the actual values. The variable close perc is again the node that is split at the root node. The other two variables which are split subsequently in the decision tree are high_perc and low_perc. The model for this case yields an RMSE value of 0.3440, while the mean of the absolute values of the actual open_perc values is 0.9286 .126 cases out of a total of 725 cases exhibit a sign mismatch between their predicted and actual open_perc values. The correlation coefficient between the actual and the predicted values of open_perc is found to be 0.96 , with the $t$-statistics value of 100.47 , and its associated $p$-value of $2.2 * 10^{-16}$. The predicted and the actual open_perc values are highly correlated. Fig. 19 shows that the regression model is effective in establishing a linear relationship between the response variable, open_perc, and the other predictor variables.

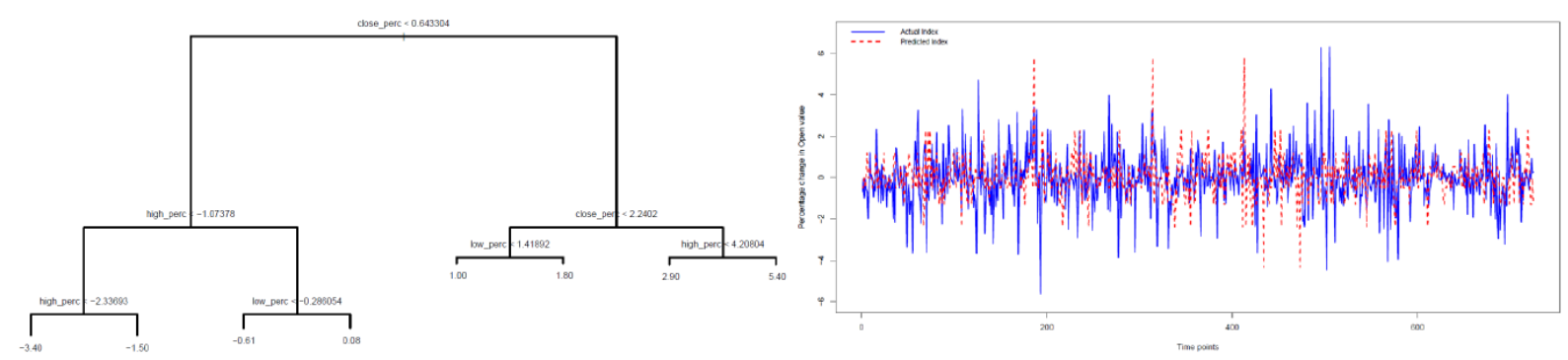

Figure 20: Decision tree regression - (a) the tree model for Case III, (b) actual and predicted open_perc for Case III
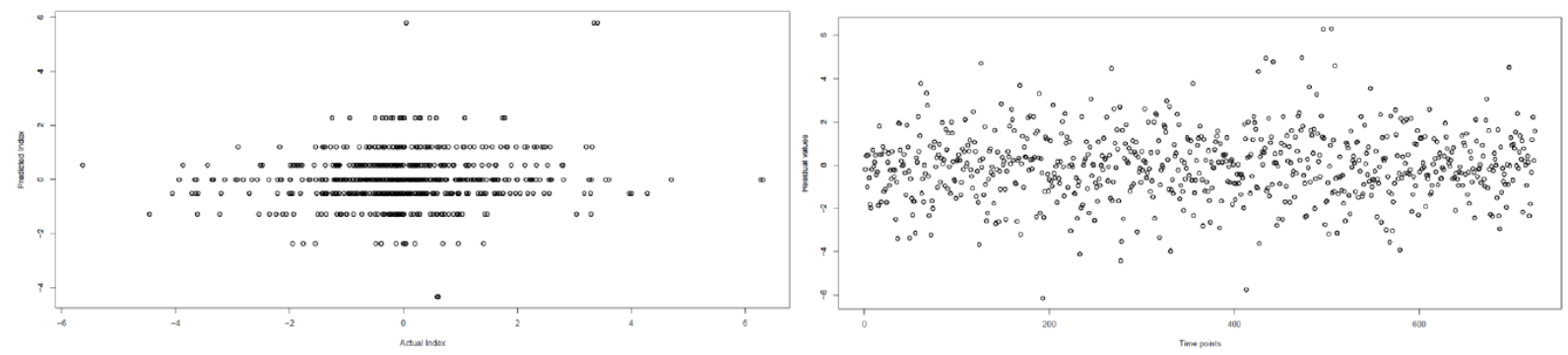

Figure 21: Decision tree regression - (a) the relationship between actual and predicted open_perc values for Case III, (b) residuals of regression for Case III

The decision tree regression model for Case III is identical to that of Case I as they are both built on the same dataset. Fig. 20 depicts the decision tree model for Case III, and the pattern of variation of the actual and predicted open_perc values for the model. Even though the model under Case III is identical to that under Case I, the performance results of the model are different. The model under Case III is tested on the 2014 data, while under Case I, the model is tested on the 2013 data. The correlation coefficient between the predicted and the actual values of open_perc for this case is found to be 0.10 , with the $t$-statistics value of 2.8243, and its associated $p$-value of 0.00487 . However, the RMSE for this case is higher than those in the previous two cases. The RMSE is found to be 1.5407, while the mean of the absolute values of the 
actual open_perc is 0.9286 . Thus, the ratio of the RMSE to the mean of the absolute values of actual open perc turns out to be 165.92 - a very high value. 346 cases out of a total of 725 cases exhibit a mismatch in sign between the predicted and the actual values of open_perc. The model is unable to make any accurate prediction as it has a very limited number of values to map into a set of a large set of continuously varying open_perc values for the year 2014.

Fig. 21 presents the performance of the model in Case III. It is evident from Fig 21 that there are significant deviations between the patterns exhibited by the actual and the predicted open_perc values. This leads to a significantly higher RMSE in this case for the decision tree model.

Table 12: Bagging regression results

\begin{tabular}{|l|c|c|c|}
\hline \multirow{2}{*}{ Metrics } & Case I & Case II & Case III \\
\cline { 2 - 4 } & Training 2013 & Training 2014 & Test 2014 \\
\hline Correlation Coefficient & 0.96 & 0.98 & 0.97 \\
\hline RMSE/Mean of Absolute Values of Actuals & 40.29 & 25.70 & 34.91 \\
\hline Percentage of Mismatched Cases & 4.70 & 5.10 & 9.24 \\
\hline
\end{tabular}

Bagging Regression: The bagging function defined in the ipred library of R programming language is used in building the bagging regression model. In Case I, the RMSE is found to be 0.2579 , with the mean of the absolute values of open_perc as 0.6402 . Out of 745 total cases, 35 cases exhibit mismatch in the predicted and the actual values of open_perc. Fig. 22 presents the performance of the bagging regression model under Case I.
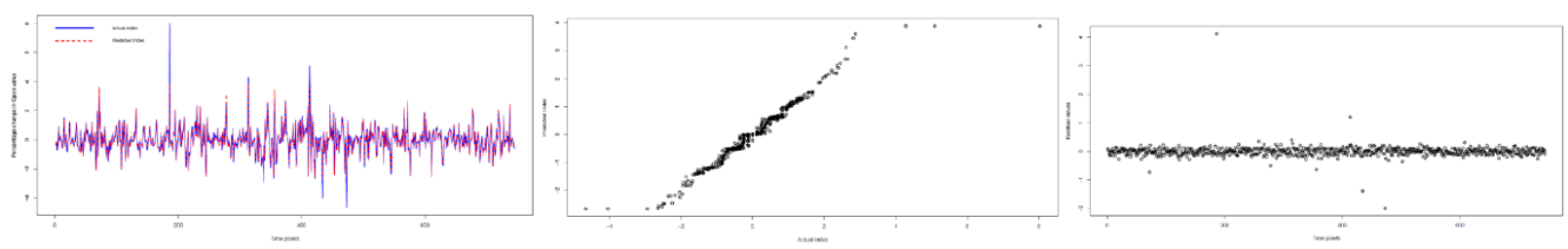

Figure 22: Bagging regression - (a) actual and predicted values of open_perc for Case I, (b) relationship between actual and predicted open_perc for Case I, (c) residuals for Case I
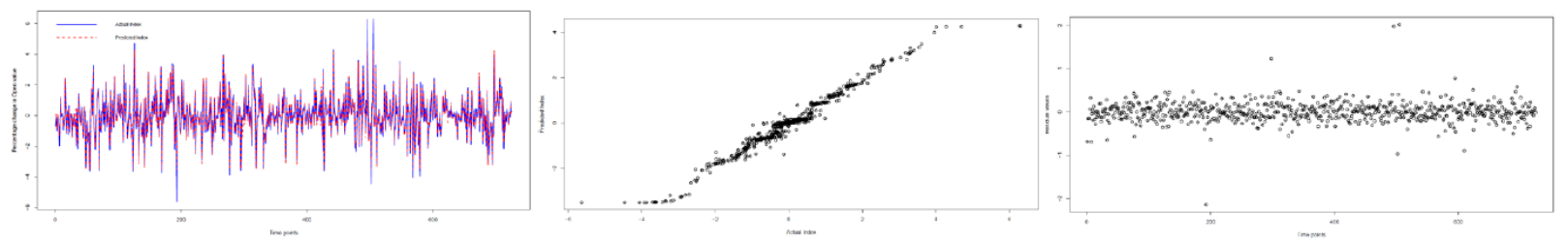

Figure 23: Bagging regression - (a) actual and predicted values of open_perc for Case II, (b) the relationship between actual and predicted open_perc for Case II, (c) residuals for Case II 
The bagging model under Case II yields an RMSE value of 0.2386 , while the mean of the absolute values of the actual open perc is 0.9286 . We find that 37 cases out of a total of 725 cases exhibit a mismatch in sign between the predicted and the actual values of open_perc. Fig. 23 depicts the performance of the bagging regression model under Case II. The RMSE value for Case III is found to be 0.3242 . We observe that 67 cases out of a total of 725 cases show a mismatch in sign between its predicted and the actual values of open_perc. The performance of the bagging model under Case III is presented in Fig. 24. Table 12 presents the overall results of the bagging regression model.

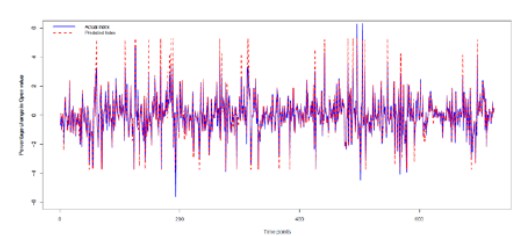

Figure 24: Bagging regression - (a) actual and predicted values of open perc for Case III, (b) the relationship between actual and predicted open_perc for Case III, (c) residuals for Case III

Table 13: Boosting regression results

\begin{tabular}{|l|c|c|c|}
\hline \multirow{2}{*}{ Metrics } & Case I & Case II & Case III \\
\cline { 2 - 4 } & Training 2013 & Training 2014 & Test 2014 \\
\hline Correlation Coefficient & 0.99 & 0.99 & 0.97 \\
\hline RMSE/Mean of Absolute Values of Actuals & 23.40 & 17.35 & 41.51 \\
\hline Percentage of Mismatched Cases & 0.81 & 4.69 & 6.90 \\
\hline
\end{tabular}
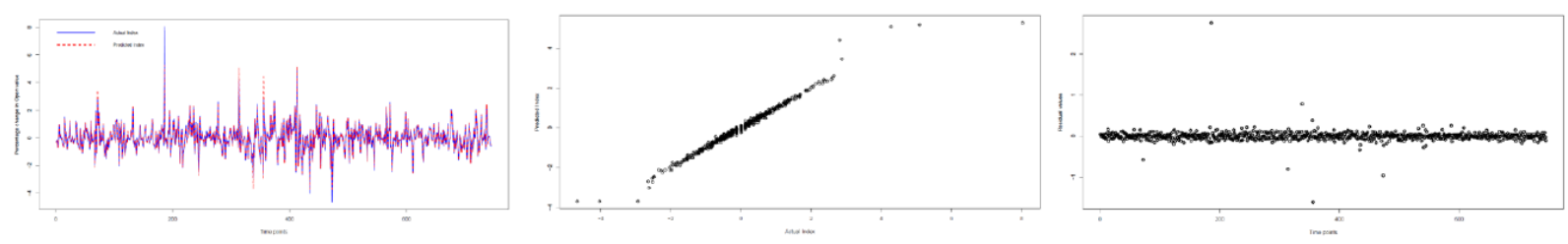

Figure 25: Boosting regression - (a) actual and predicted values of open perc for Case I, (b) relationship between actual and predicted open_perc for Case I, (c) residuals for Case I

Boosting Regression: We use the blackboost function defined in the mboost library in the R programming language for building the boosting regression models. In Case I, 6 cases out of 745 cases exhibit mismatched signs between the predicted and the actual open_perc values. The RMSE for this case is found to be 0.1498 , while the mean of the absolute values of the actual open perc is 0.6402 . The performance of the boosting regression model under Case I is presented in Fig. 25.
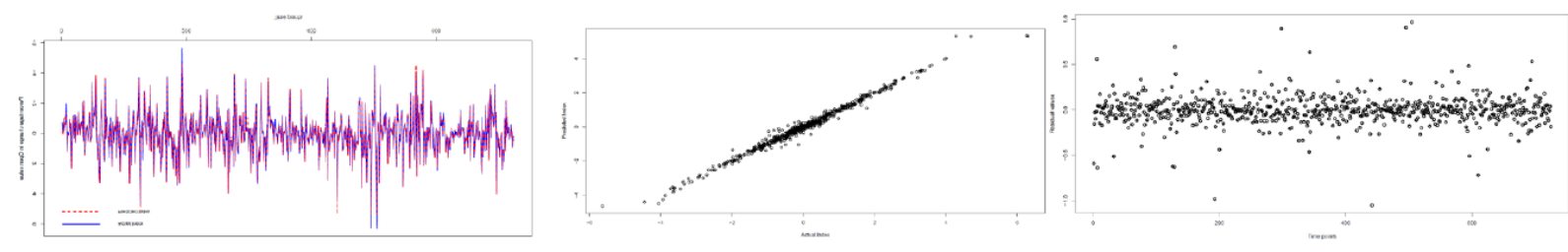
Figure 26: Boosting regression - (a) actual and predicted values of open_perc for Case II, (b) the relationship between actual and predicted open_perc for Case II, (c) residuals for Case II

For Case II, out of 725 total cases, 34 cases yield mismatched signs between the actual and the predicted values of open_perc. The RMSE for this case is 0.1611 , and the mean of the absolute values of the actual open_perc is 0.9286. The performance of the boosting model under Case II is presented in Fig. 26.

Case III yields an RMSE value of 0.3855 , while the mean of the absolute values of the actual open_perc is 0.9286. In Case III, 50 cases out of a total of 725 cases exhibit mismatched signs between the predicted and the actual open_perc values. The performance of the boosting model under Case III is presented in Fig. 27. Table 13 presents the results of the boosting regression model.

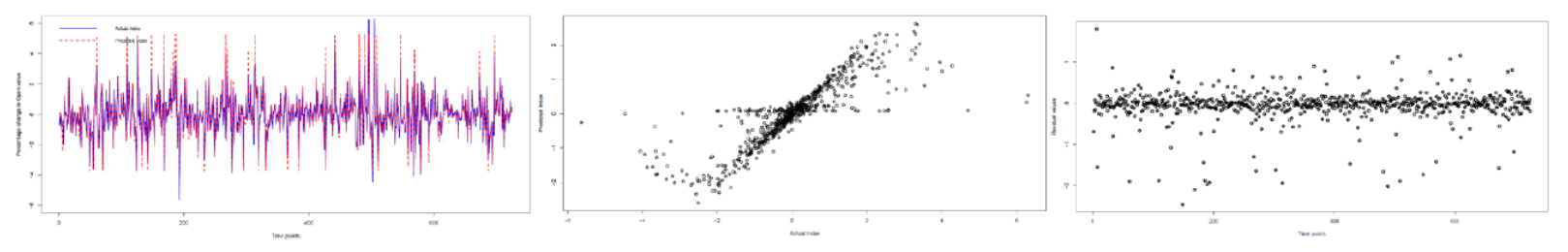

Figure 27: Boosting regression - (a) actual and predicted values of open_perc for Case III, (b) the relationship between actual and predicted open_perc for Case III, (c) residuals for Case III

Table 14: Random Forest regression results

\begin{tabular}{|l|c|c|c|}
\hline \multirow{2}{*}{ Metrics } & Case I & Case II & Case III \\
\cline { 2 - 4 } & Training 2013 & Training 2014 & Test 2014 \\
\hline Correlation Coefficient & 0.99 & 0.99 & 0.97 \\
\hline RMSE/Mean of Absolute Values of Actuals & 16.26 & 10.82 & 32.02 \\
\hline Percentage of Mismatched Cases & 0.00 & 2.62 & 6.48 \\
\hline
\end{tabular}
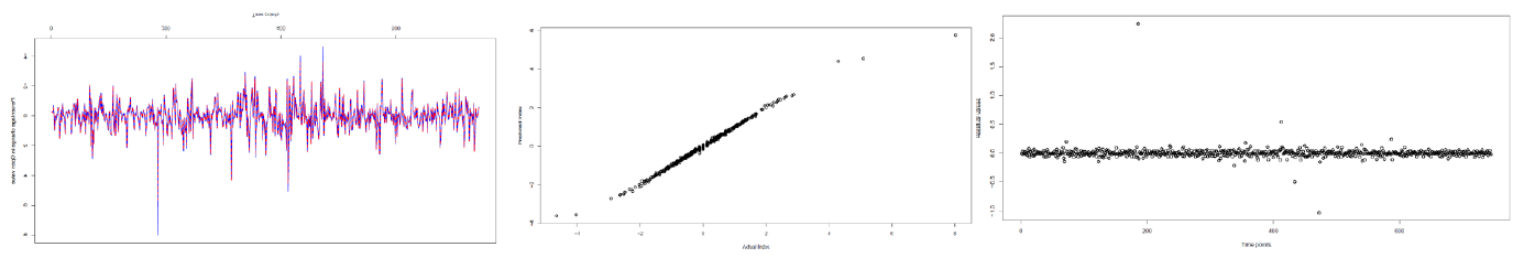

Figure 28: Random forest regression - (a) actual and predicted values of open_perc for Case I, (b) relationship between actual and predicted open_perc for Case I, (c) residuals for Case I

Random Forest Regression: We use the randomForest function defined in the randomForest library in the $\mathrm{R}$ programming language for building the random forest regression model. For all three cases, the algorithm tried using three variables at each split of the associated decision tree. The number of regression decision trees constructed in each case is 500 . The mean squared residual values are found to be $0.0441,0.0512$, and 0.0441 respectively for Case I, Case II, and Case III respectively. In Case I, the percentage of variance explained by the model is 95.13 . None of the 745 cases exhibit any sign mismatch between the predicted and the actual values of open perc. While the RMSE for this case is 0.1041 , the mean of the absolute values of the actual open_perc is 0.6402 . For Case II, the model can explain $97.11 \%$ of the variance, and 
19 cases out of a total of 725 cases exhibit mismatched signs between the predicted and the actual open_perc values. The RMSE for this case is 0.1005 , while the mean of the absolute values of the actual open_perc is 0.9286 . In Case III the model can explain $95.13 \%$ of the variance of the response variable. It is observed that 47 cases out of 725 cases exhibit mismatched signs between the predicted and the actual open_perc values. The RMSE is 0.2973 , while the mean of the absolute values of the actual open_perc values is 0.9286 . Table 14 presents the results of the random forest regression model.

Fig. 28 depicts the performance results of the random forest regression model under Case I. Fig. 28(a) depicts the pattern of variation of the predicted open_perc values vis-a-vis their corresponding actual values for each of the 745 time-slots in Case I. The linear relationship between the predicted and the actual open_perc values is presented in Fig 28(b). The residual values for the regression model are depicted in Fig. 28(c). These three visuals along with the results presented in Table 14 indicate that the random forest regression for Case $I$ is very accurate.
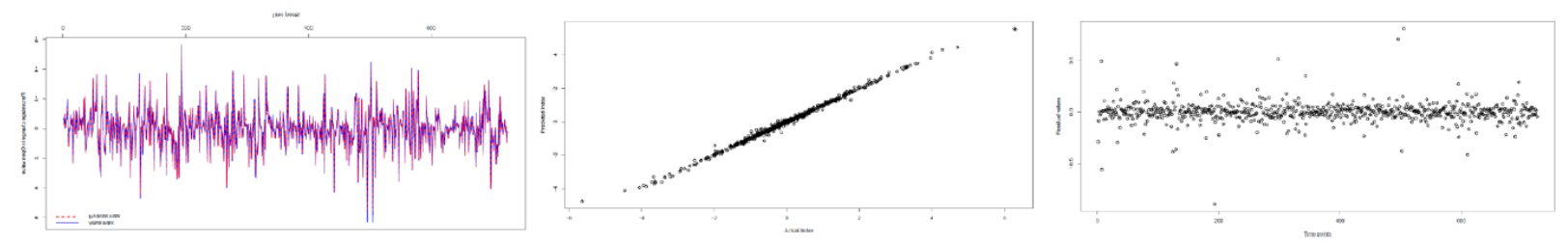

Figure 29: Random forest regression - (a) actual and predicted values of open_perc for Case II, (b) relationship between actual and predicted open_perc for Case II, (c) residuals for Case II

Fig. 29 presents the graphical representations of the performance results of the random forest regression model for Case II. It is evident that the predicted values of open_perc very closely resemble the patterns exhibited by the actual values. Moreover, the residuals of the regression model exhibit randomness, and there is no evidence of any autocorrelations in the residuals.
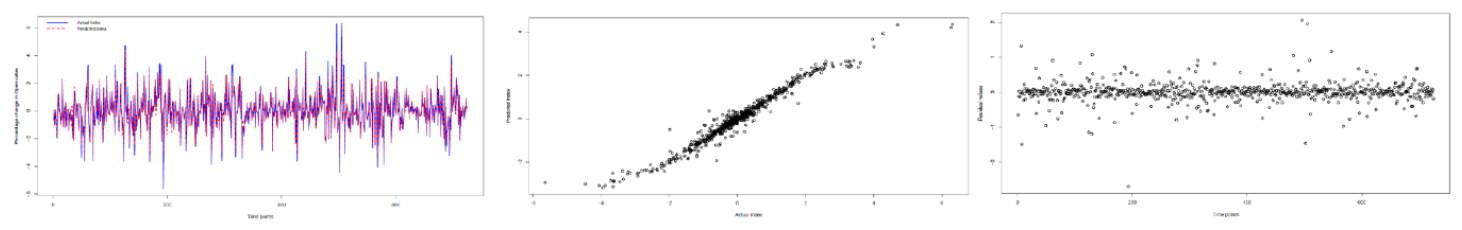

Figure 30: Random forest regression - (a) actual and predicted values of open_perc for Case III, (b) relationship between actual and predicted open_perc for Case III, (c) residuals for Case III

The performance results of the random forest regression model are resented in Fig. 30. The results indicate that the random forest regression model for Case III is accurate. Fig 30(b) exhibits some deviations from linearity at the head and the tail portions of the graph, while the middle part of the segment depicts a strong linear pattern between the actual and the predicted values of open_perc. However, the deviations from the linear pattern results in a marginally higher value for the ratio of the RMSE to the mean of the absolute values of open_perc under Case III. 
ANN Regression: We use the neuralnet function defined in the neuralnet library in the R programming language for designing the ANN regression models. For Case I, 9 out of 745 records are found to exhibit mismatched signs between the actual and the predicted open_perc values. The RMSE, in this case, is found to be 0.1099 , and the mean of the absolute values of the actual open_perc values is 0.6402 . In Case II, it is found that 68 out of 725 cases exhibit a mismatch in sign between their actual and predicted open_perc values. The RMSE of the model for Case II is found to be 0.2915. In Case III, we find that 79 out of 725 cases exhibit a mismatch in sign between the predicted and the actual values of open_perc. The RMSE of the model for this case is found to be 0.3420 . The results for the ANN regression model are presented in Table 15.

Table 15: ANN regression results

\begin{tabular}{|l|c|c|c|}
\hline \multirow{2}{*}{ Metrics } & Case I & Case II & Case III \\
\cline { 2 - 4 } & Training 2013 & Training 2014 & Test 2014 \\
\hline Correlation Coefficient & 0.99 & 0.98 & 0.98 \\
\hline RMSE/Mean of Absolute Values of Actuals & 17.16 & 31.39 & 36.83 \\
\hline Percentage of Mismatched Cases & 1.21 & 9.38 & 10.90 \\
\hline
\end{tabular}
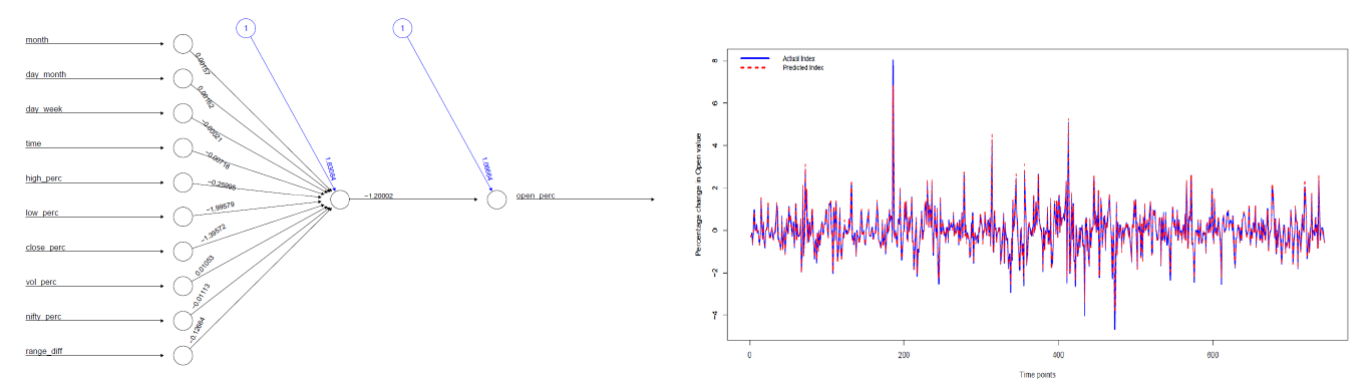

Figure 31: ANN regression - (a) the model for Case I, (b) actual and predicted open_perc for Case I
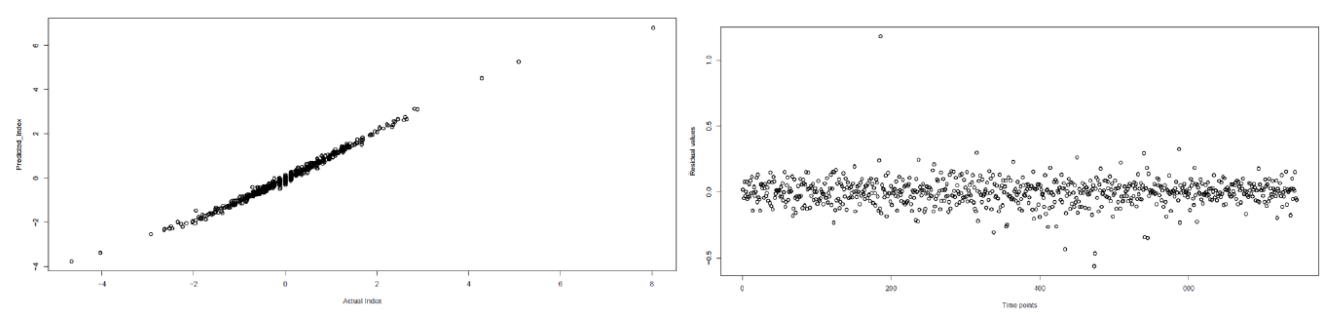

Figure 32: ANN regression - (a) the relationship between actual and predicted open_perc for Case I, (b) residuals for Case I

Fig. 31 depicts the ANN regression model for Case I, and the pattern of variation of actual and predicted values of open_perc. Only one node is used in the hidden layer as it is found to be sufficient to model the nonlinearity in the data. In the figure, the link weights are marked in black color, while the bias values associated with the hidden layer node and the output layer node are written in blue color. The nodes in the input layer correspond to the input variables of the model. Fig. 32 exhibits the functional relationship between the predicted and the actual values of open_perc, and the residuals of the regression model. It is evident that the residuals are random, and they do not exhibit any significant autocorrelation. For Case I, 
the correlation coefficient between the actual and the predicted values of open_perc is 0.99 , and the percentage of cases that exhibit sign mismatch between the predicted and the actual open_perc is 1.07.
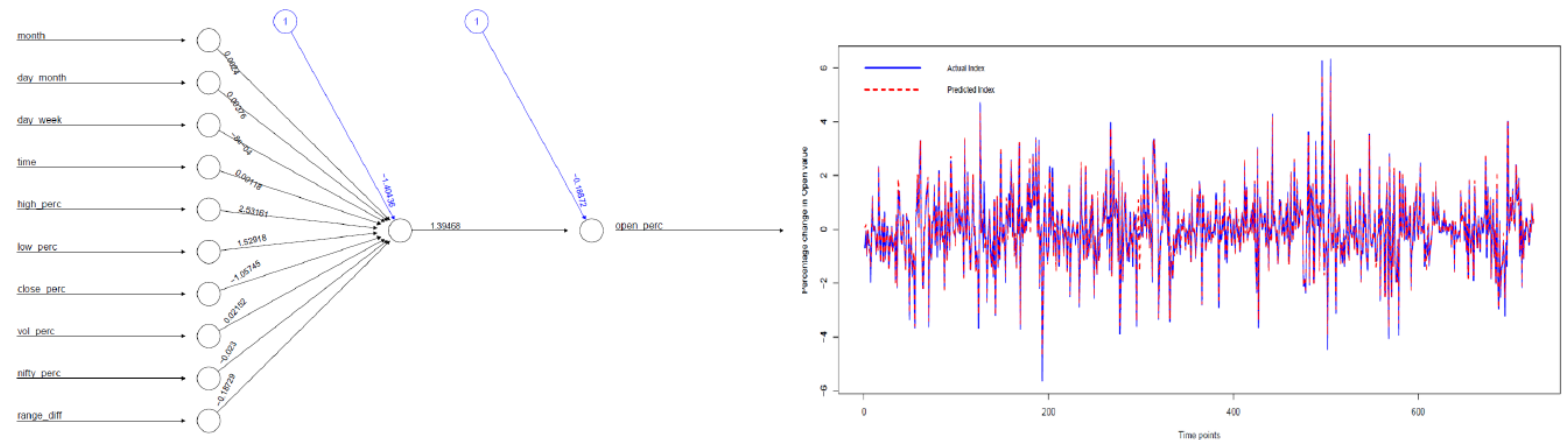

Figure 33: ANN regression - (a) the model for Case II, (b) actual and predicted open_perc for Case II
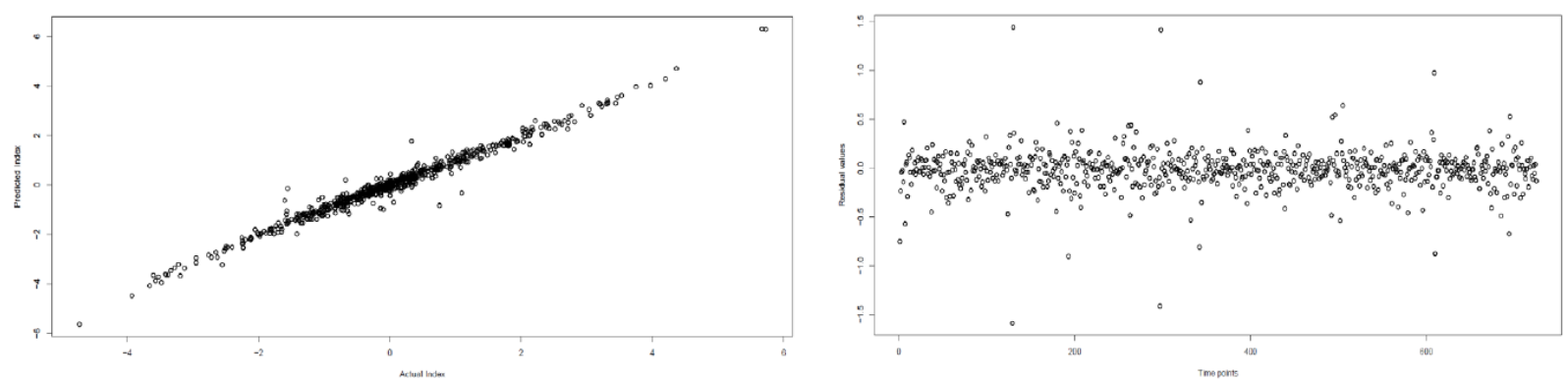

Figure 34: ANN regression - (a) the relationship between actual and predicted open_perc for Case II, (b) residuals for Case II

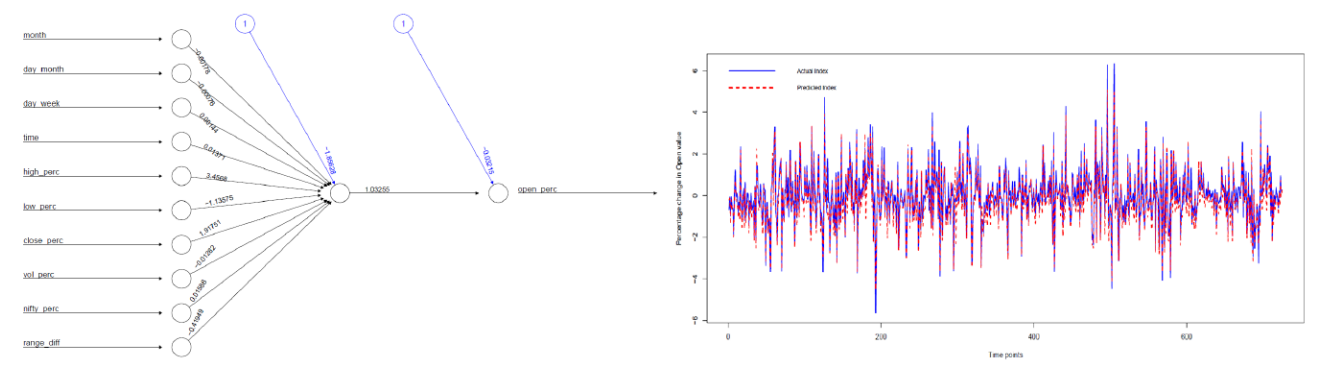

Figure 35: ANN regression - (a) the model for Case III, (b) actual and predicted open_perc for Case III

Fig. 33 shows the ANN regression model for Case II, and the pattern of variation of the actual and the predicted values of open_perc for this model. It is evident that the predicted values of open_perc very closely follow the actual open_perc values. Fig. 34 depicts that there is a high degree of linearity in the relationship between the predicted and the actual values of open_perc, and the residuals of the regression model are free from any significant autocorrelation. 

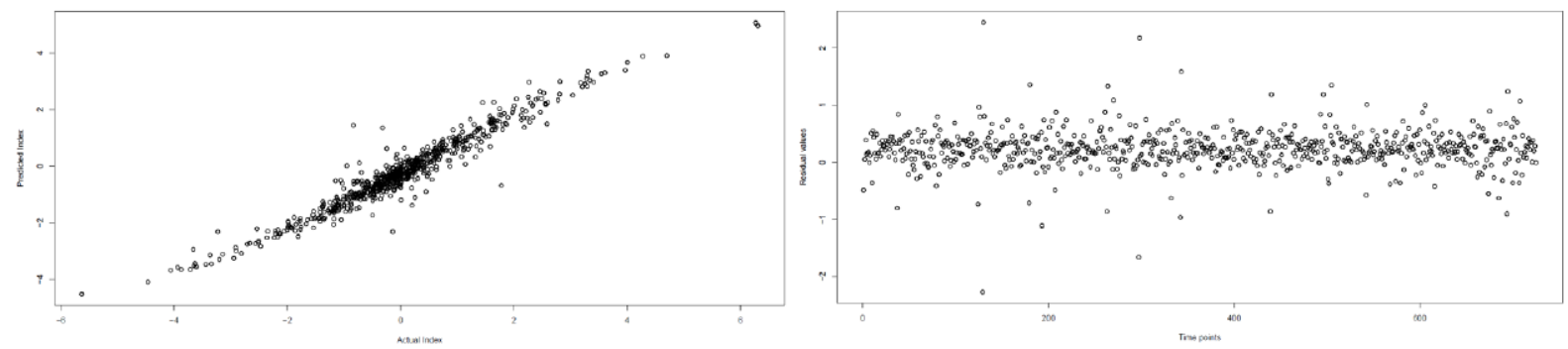

Figure 36: ANN regression - (a) the relationship between actual and predicted open_perc for Case III, (b) residuals for Case III

Fig, 35 and Fig. 36 depict the ANN regression model for Case III and its various performance results. These figures and the values of the numerical metrics like correlation coefficient, the ratio of RMSE to the mean of the absolute values of the actual open_perc, and the number sign mismatch cases between the predicted and the actual values of open_perc, all show that the model is very accurate in its task of prediction.

Table 16: SVM regression results

\begin{tabular}{|l|c|c|c|}
\hline \multirow{2}{*}{ Metrics } & Case I & Case II & Case III \\
\cline { 2 - 4 } & Training 2013 & Training 2014 & Test 2014 \\
\hline Correlation Coefficient & 0.93 & 0.98 & 0.83 \\
\hline RMSE/Mean of Absolute Values of Actuals & 53.88 & 27.92 & 82.96 \\
\hline Percentage of Mismatched Cases & 0.27 & 4.41 & 13.19 \\
\hline
\end{tabular}

SVM Regression: We use the svm function defined in the e1071 library of the R programming language. For all three cases, the regression type used is eps-regression, and the kernel is radial. The values of the parameters gamma and epsilon are both found to be 0.1 . The algorithm finds the number of support vectors as 248, 265, and 246 for Case I, Case II, and Case III respectively. The RMSE values for the three cases are found to be $0.3450,0.2593$, and 0.7703 respectively. The mean of the absolute values of the open_perc is 0.6402 . We compute the ratio of the RMSE to the mean of the absolute values of open_perc for all three cases. We also identify the cases which exhibit a difference in the signs between the actual and the predicted values of open_perc. These are the cases, in which the regression model fails to predict the direction of the movement of the actual open_perc values.

For Case I, 2 out of 745 cases are found to exhibit a sign mismatch between the actual and the predicted values of open_perc. 32 out of 725 cases yield sign mismatch in Case II. In Case III, since the model encounters a more difficult challenge in prediction, 95 out of 725 cases are mismatched in sign. The SVM regression results are presented in Table 16. For all three cases, the SVM regression model looks quite accurate in its results.

Fig. 37 presents the performance results of the SVM regression model under Case I. It is evident from Fig. 37 (a) that for this model, the predicted series very closely follows the pattern of the variation in the actual open_perc values. Fig. 37 (b) clearly shows that except for some points at the tail and the head, most of the 
points exhibit a strong linear relationship between the actual and the predicted values of open_perc for Case I. The residual plot in Fig. 37(c) shows that most of the residuals values are random.
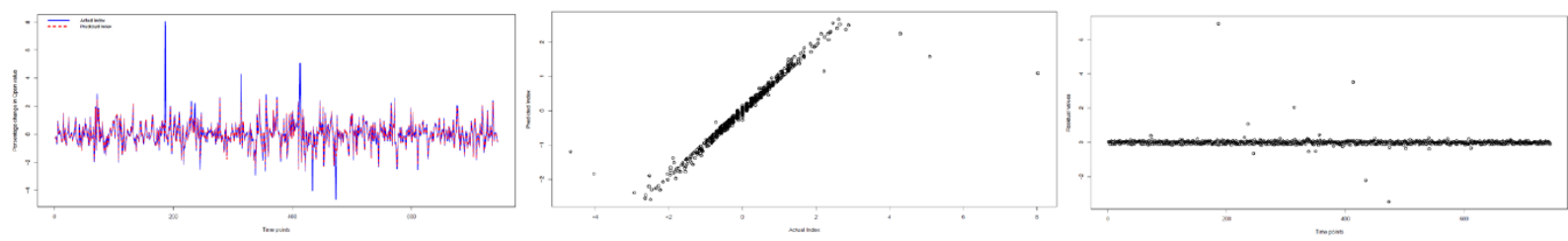

Figure 37: SVM regression - (a) actual and predicted values of open_perc for Case I, (b) relationship between actual and predicted open_perc for Case I, (c) residuals for Case I
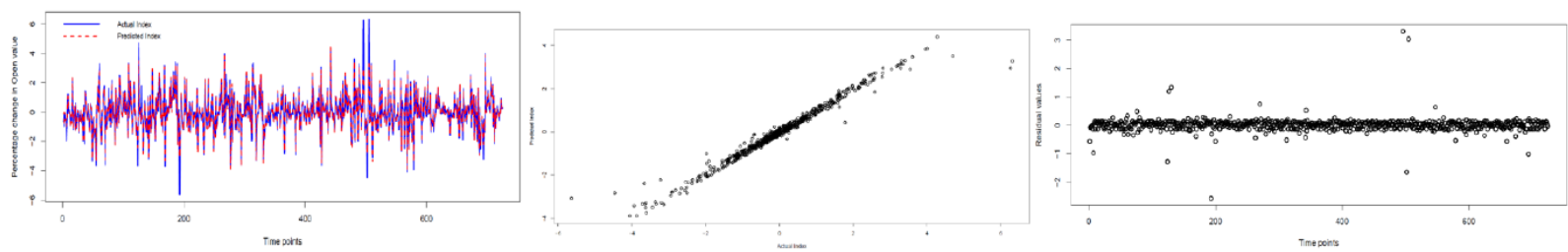

Figure 38: SVM regression - (a) actual and predicted values of open_perc for Case II, (b) the relationship between actual and predicted open_perc for Case II, (c) residuals for Case II

The performance of the SVM model in Case II is very similar to that in Case I. This is evident from Fig. 38. These figures exhibit similar patterns shown in Fig. 37. However, it is found that the ratio of the RMSE to the mean of the absolute values of the actual open_perc is smaller in Case II than that in Case I.
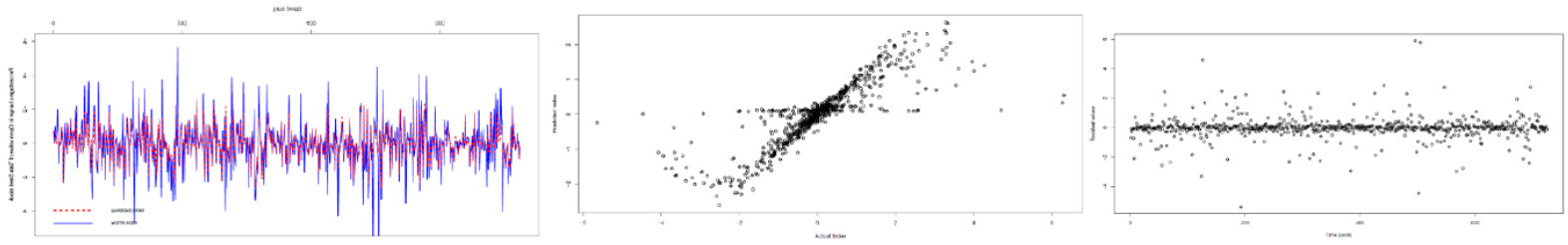

Figure 39: SVM regression - (a) actual and predicted values of open_perc for Case III, (b) the relationship between actual and predicted open_perc for Case II, (c) residuals for Case III

However, it is observed from Fig. 39 that Case III poses a more difficult challenge to the SVM regression model. For Case III, the correlation coefficient between the actual and the predicted values of the open_perc is found to be much smaller in comparison to the other two cases. Fig. 39(a) shows that the predicted values of open_perc do not exactly follow the pattern of variation exhibited by the actual open_perc values. It is also evident from Fig. 39(b) that there is a high degree of nonlinearity between the predicted and the actual open_perc values. However, no evidence of any significant autocorrelations between the residuals are observed in Fig. 39(c).

LSTM Regression: In Section 5, we briefly presented a brief overview of LSTM networks. In the following, we present, the results of the LSTM-based regression models. These are the sequential steps that are followed in building the LSTM models: (i) reading the file containing the raw data, (ii) normalizing the 
data, (iii) converting the normalized data into a time series and then into a supervised learning problem, (v) creating a deep learning model using Tensorflow and Keras frameworks, (vi) training and validating the model, (vii) visualization of the training and validation performance, and (viii) evaluating the accuracy of the model on test data. The raw data consists of the following attributes: (i) year, (ii) month, (iii) day, (iv) hour (i.e., the time slot), (v) open, (vi) high, (vii) low, (viii) close, (ix) volume, and (x) the NIFTY index. Using Python programming, we combine the attributes year, month, day, and hour into a single attribute, and use the combined new attribute as the index. Other remaining attributes are now used for building the models. We provide the details of the three cases in the following.
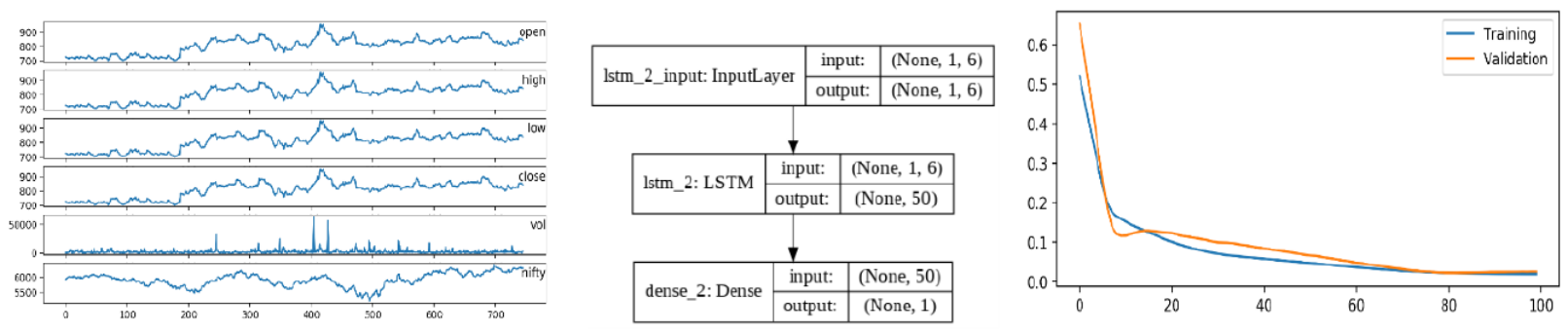

Figure 40: LSTM regression - (a) the representation of the stock price data for Case I, (b) the model architecture for Case I, Case II, and Case III, and (c) the training and validation losses for Case I

For Case I, we first plot the open, high, low, close, volume, and the NIFTY time series. In this case, there are 746 records. Fig. 40(a) depicts the time series for each of the attributes. All the six value attributes (leaving out the index column) are then normalized using the MinMaxScalar function defined in the processing submodule of the sklearn module in Python. Out of the total 746 records, the first 500 records are used for training the model while the remaining 246 records are left out for validation. The Sequential function defined in Keras is used for building the layers and the model is compiled using mean absolute error (MAE) as the loss function and adaptive moment estimation (Adam) as the optimizer. The model architecture is presented in Fig. 40(b). In this model, the input layer consists of six attributes, and the input data is passed on to the LSTM layer that has 50 nodes so that 50 features are extracted from the input data. The output of the LSTM layer is passed on to a dense layer (i.e., a fully connected layer) that has 1 node at its output which produces the next predicted value of the time series. The behavior of the training and the validation loss values is studied for different values of epochs and batch sizes. With a batch size of 72 and an epoch value of 100 , the training and validation losses are found to have converged to a very low value. Fig. 40(c) presents the behavioral patterns of the training and the validation losses in Case I. On the completion of the final epoch, the RMSE value is 8.812, and the correlation coefficient between the actual and the predicted open values is 0.983 . The training and validation loss values are found to be 0.0194 and 0.0252 respectively.

Case II involves slot-wise stock prices for the year 2014, and there are 725 records in the dataset in this case. Fig. 41(a) presents the plots for the time series attributes for Case II. In this case too, before building the model, the raw values of the six attributes are normalized using the MinMaxScalar function. The model architecture remains identical to that of Case I, which is represented in Fig. 40(b). For training the model, the first 500 records are used, while the remaining 225 records are used for the model validation. It is observed that, initially, the validation loss converges with the training loss at an epoch value of 40 . 
However, it starts increasing again with the increase in the epoch value. The validation loss finally converges with the training loss at an epoch value of 100 when a batch of 72 is used. The RMSE of the model is found to be 15.002, and the correlation coefficient between the actual and the predicted open values is 0.982 . On completion of the final epoch, the training and the validation loss are found to be 0.0134 and 0.0301 respectively. Fig. 41(b) depicts the convergence of the training and the validation loss in Case II.
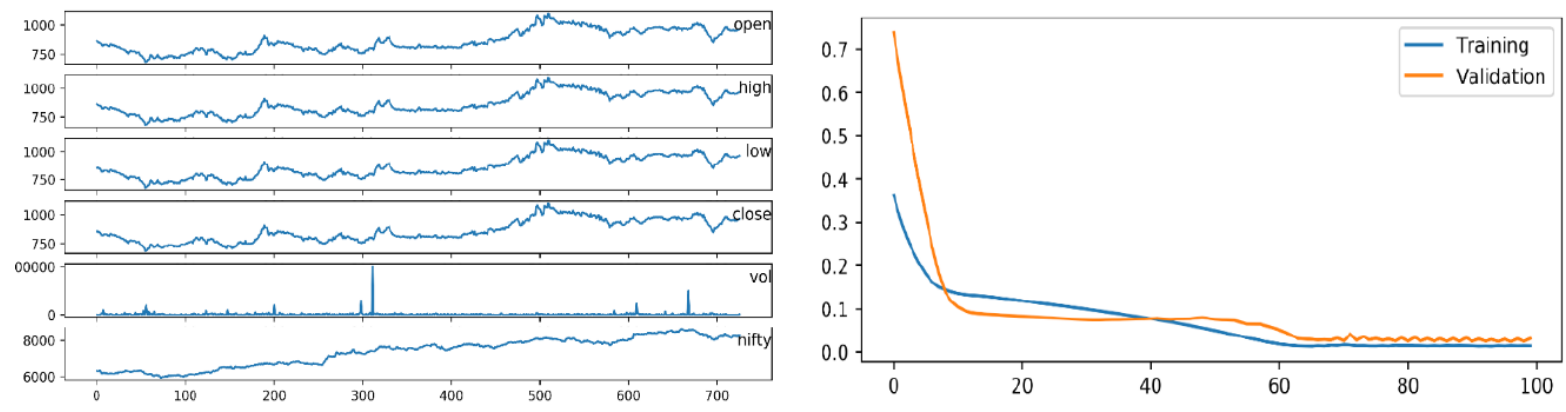

Figure 41: LSTM regression - (a) the representation of the stock price data for Case II, (b) the training and validation losses for Case II
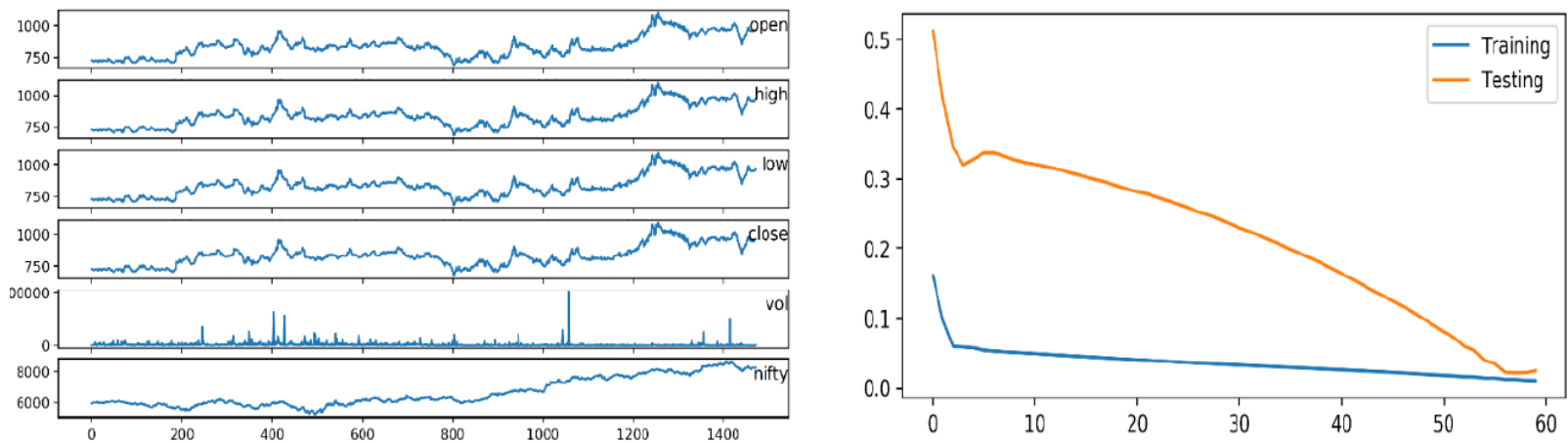

Figure 42: LSTM regression - (a) the representation of the stock price data for Case III, (b) the training and validation losses for Case III

In Case III, the LSTM model is built using data of the year 2013, and then the model is tested on the stock price records of the year 2014. The raw dataset, in this case, consists of 1471 records in total - 746 records (those belonging to the year 2013) are used in building the model, and the remaining 725 records (those belonging to the year 2014) are used for testing the model. Fig 42(a) presents the plots of the open, high, low, close, volume, and the NIFTY time series for the 1471 records. The LSTM model architecture remains unchanged and is identical to that of Case I. The training and the test losses converge with each other after an epoch value of 60 with a batch size of 72 . The RMSE and the correlation coefficient, for this case, are found to be 13.477 and 0.996 respectively. After the completion of the last epoch, the training and the test losses are 0.0116 and 0.0258 respectively. Fig, 42(b) depicts the convergence of the training and the testing losses with varying epoch values for Case III. 
CNN Regression: In Section 5, we presented a brief overview of how CNN regression models are used to carry out multi-step forecasting of the open values of the stock price time series. Unlike the machine learning and the LSTM models, we follow a different approach in model building testing for the CNN models. We use the stock price data from December 31, 2012 (a Monday) to January 9, 2015 (a Friday) for our analysis. The original stock price dataset consists of records that are collected at 5 minutes interval of time. For each time slot the values of open, high, low, close, and volume are available. The stock price data from December 31, 2012, to December 30, 2013, is used for training, while the models are tested on the data from December 31, 2013 to January 9, 2015. The time-series data is also arranged in the form of a repetitive weekly sequence from Monday to Friday. Fig. 43 depicts the time series data of stock prices at 5 minutes. As mentioned in Section 5, we follow four different models of CNN regression. The models are as follows.

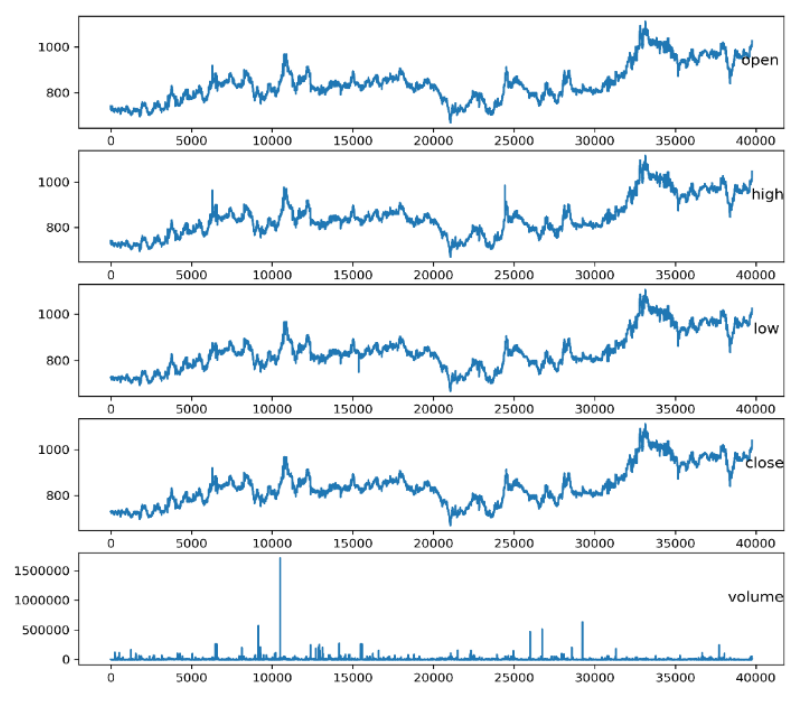

Figure 43: The stock price data of Godrej Consumer Ltd. at five minutes interval during 2013 - 2015

CNN Model \#1: This model is based on a univariate forecasting approach with the previous one week's data as the input. Since is a week consists of five data points, the input size of the model is five. Since the input data size is small, we build a light CNN model for this case. One convolution layer with 16 filters and a kernel size of 3 is used. In other words, it means that the input sequence of five days is read with a convolutional operation in three time-steps at a time, and this operation is performed 16 times. A maxpooling layer of size 2 is used that reduces the size of the feature maps before the internal representation is flattened to one long vector. This is interpreted by a fully-connected layer before the output layer (which is also fully connected) predicts the open values for the next five days. Fig. 44(a) depicts the architecture of the CNN model \#1. In the convolution layer and the fully connected layer, the rectified linear unit (ReLU) function is used as the activation function. The Adam optimizer is used in the stochastic gradient descent algorithm, and 20 epochs with a batch size of 4 are used for building and compiling the model. The mean squared error (MSE) is used as the loss function at the output layer. For computing the error in prediction, root mean squared error (RMSE) is used as the metric. With a small batch size, and with the use of the 
stochastic nature of the gradient descent algorithm, the model is expected to learn a slightly different mapping of the inputs to the outputs every time it is trained. This implies that performance results will vary slightly in different runs. Hence, the model is iterated over several rounds and the mean values of the metrics are taken as the overall performance of the model.

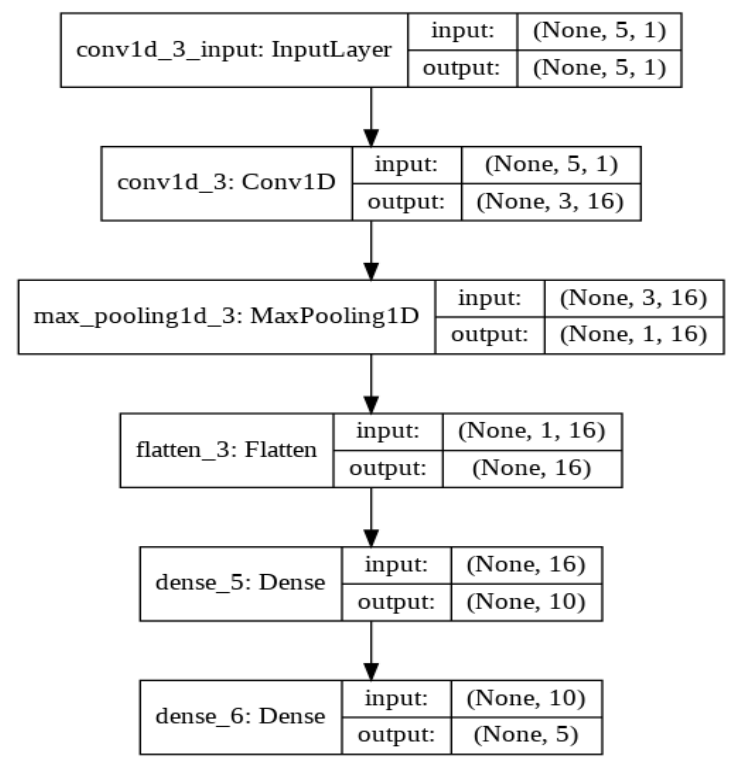

\begin{tabular}{|c|c|c|c|c|c|c|c|}
\hline Round_No & Overall RMSE & Monday & Tuesday & Wednesday & Thursday & Friday & Exec_Time \\
\hline 1 & 5.368 & 4.6 & 4.7 & 5.4 & 5.6 & 6.2 & 79.795 \\
\hline 2 & 5.482 & 3.9 & 4.8 & 5.8 & 5.6 & 6.9 & 80.419 \\
\hline 3 & 6.074 & 5.3 & 5.3 & 6.5 & 6.1 & 6.9 & 86.294 \\
\hline 4 & 5.647 & 4.7 & 5.4 & 6.0 & 6.0 & 6.1 & 85.200 \\
\hline 5 & 5.226 & 3.8 & 4.7 & 5.3 & 6.0 & 6.0 & 84.000 \\
\hline 6 & 5.340 & 3.8 & 4.7 & 5.5 & 6.1 & 6.2 & 81.377 \\
\hline 7 & 5.357 & 3.7 & 5.2 & 5.8 & 5.7 & 6.0 & 81.940 \\
\hline 8 & 6.018 & 3.9 & 5.1 & 6.1 & 7.4 & 6.9 & 81.626 \\
\hline 9 & 5.121 & 3.7 & 4.7 & 5.4 & 5.6 & 6.0 & 80.998 \\
\hline 10 & 7.019 & 6.0 & 7.2 & 6.4 & 6.6 & 8.6 & 82.542 \\
\hline 11 & 6.657 & 5.9 & 6.6 & 6.5 & 6.8 & 6.9 & 84.902 \\
\hline 12 & 6.598 & 4.9 & 7.5 & 7.0 & 6.3 & 6.9 & 82.832 \\
\hline 13 & 5.364 & 3.9 & 4.9 & 5.4 & 5.9 & 6.4 & 81.955 \\
\hline 14 & 5.371 & 3.9 & 4.7 & 5.7 & 5.7 & 6.5 & 83.065 \\
\hline 15 & 5.769 & 5.2 & 6.0 & 5.8 & 5.7 & 6.2 & 79.991 \\
\hline 16 & 5.470 & 4.7 & 5.2 & 5.9 & 5.6 & 5.9 & 98.986 \\
\hline 17 & 5.392 & 4.9 & 5.0 & 5.3 & 5.6 & 6.0 & 80.584 \\
\hline 18 & 5.704 & 4.6 & 5.5 & 6.2 & 6.0 & 6.1 & 79.428 \\
\hline 19 & 5.169 & 3.8 & 4.7 & 5.4 & 5.7 & 6.0 & 78.198 \\
\hline 20 & 5.636 & 4.5 & 5.3 & 5.8 & 5.7 & 6.6 & 79.228 \\
\hline Mean & $\mathbf{5 . 6 8 9 1}$ & $\mathbf{4 . 4 8 5}$ & $\mathbf{5 . 3 6}$ & $\mathbf{5 . 8 6}$ & $\mathbf{5 . 9 8 5}$ & $\mathbf{6 . 4 6 5}$ & 82.668 \\
\hline SD & $\mathbf{0 . 5 3 0}$ & $\mathbf{0 . 7 2 3}$ & $\mathbf{0 . 8 3 8}$ & $\mathbf{0 . 4 7 3}$ & $\mathbf{0 . 4 7 7}$ & $\mathbf{0 . 6 2 4}$ & $\mathbf{4 . 3 9 2}$ \\
\hline Min & $\mathbf{5 . 1 2 1}$ & $\mathbf{3 . 7}$ & $\mathbf{4 . 7}$ & $\mathbf{5 . 3}$ & $\mathbf{5 . 6}$ & $\mathbf{5 . 9}$ & $\mathbf{7 8 . 1 9 8}$ \\
\hline Max & $\mathbf{7 . 0 1 9}$ & $\mathbf{6}$ & $\mathbf{7 . 5}$ & $\mathbf{7}$ & $\mathbf{7 . 4}$ & $\mathbf{8 . 6}$ & $\mathbf{9 8 . 9 8 6}$ \\
\hline RMSE/Mean & $\mathbf{0 . 0 0 6 5}$ & $\mathbf{0 . 0 0 5 2}$ & $\mathbf{0 . 0 0 6 2}$ & $\mathbf{0 . 0 0 6 8}$ & $\mathbf{0 . 0 0 6 9}$ & $\mathbf{0 . 0 0 7 5}$ & \\
\hline & & & & & & & \\
\hline
\end{tabular}

Figure 44: The CNN Model \#1 - (a) the model architecture, and (b) regression results of the univariate time series model with previous one week's data as the input

We test the model for 20 rounds and note the performance of the model in each round for its overall RMSE, the RMSE values for the individual days of a week (i.e., Monday - Friday), the execution time of the model, and the ratio of the RMSE to the mean value of the variable predicted (i.e., mean of the open value for the test dataset). Figure 44(b) presents the results for the performance of the CNN model for Case I. The mean value of open for the test data is 866.5875 . The training and the test data consist of 19500 and 20250 records respectively. The execution time for the model is expressed in seconds. The model is executed on a system consisting of an Intel i7 CPU with a clock speed of $2.60 \mathrm{GHz}-2.59 \mathrm{GHz}$ and $16 \mathrm{~GB}$ random access memory (RAM) running on the Windows 10 operating system.

CNN Model \#II: This model is based on univariate forecasting with the previous two week's data as the input. The architecture of this model is identical to that of CNN Model \#I. However, this model is fed with two weeks' prior data (i.e., 10 immediate past open values) to forecast the open values of the next week. Fig. 45(a) depicts the architecture of the CNN model for Case II. The performance results of the CNN Model \#II for 20 rounds of its execution are presented in Fig. 45(b). 


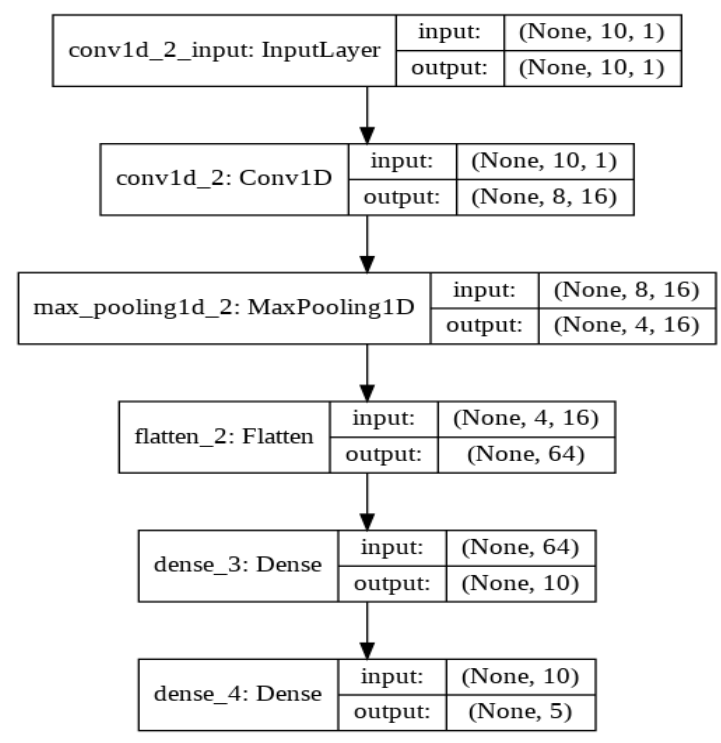

\begin{tabular}{|c|c|c|c|c|c|c|c|}
\hline Round_No & Overall RMSE & Monday & Tuesday & Wednesday & Thursday & Friday & Exec_Time \\
\hline 1 & 5.307 & 3.9 & 4.6 & 5.6 & 5.7 & 6.4 & 85.386 \\
\hline 2 & 6.042 & 3.9 & 6.2 & 6.4 & 6.8 & 6.6 & 85.845 \\
\hline 3 & 5.378 & 4.0 & 5.0 & 5.6 & 5.6 & 6.3 & 84.247 \\
\hline 4 & 5.278 & 3.5 & 4.6 & 5.5 & 5.9 & 6.3 & 108.214 \\
\hline 5 & 5.592 & 4.4 & 5.0 & 5.7 & 6.0 & 6.7 & 88.994 \\
\hline 6 & 8.852 & 6.6 & 8.7 & 9.6 & 10.4 & 8.5 & 83.484 \\
\hline 7 & 5.294 & 3.8 & 5.3 & 5.5 & 5.7 & 6.0 & 85.899 \\
\hline 8 & 6.061 & 4.7 & 4.9 & 5.9 & 6.8 & 7.5 & 88.373 \\
\hline 9 & 5.229 & 3.9 & 4.9 & 5.4 & 5.8 & 5.8 & 88.297 \\
\hline 10 & 5.857 & 5.6 & 5.3 & 5.3 & 5.9 & 7.1 & 84.636 \\
\hline 11 & 5.227 & 4.8 & 4.7 & 5.6 & 5.4 & 5.7 & 101.964 \\
\hline 12 & 8.797 & 7.8 & 8.4 & 9.0 & 9.5 & 9.1 & 87.828 \\
\hline 13 & 7.190 & 5.3 & 6.6 & 7.9 & 8.5 & 7.2 & 81.649 \\
\hline 14 & 5.697 & 4.9 & 5.7 & 5.8 & 5.9 & 6.1 & 84.072 \\
\hline 15 & 5.314 & 3.6 & 4.5 & 5.6 & 6.3 & 6.1 & 87.396 \\
\hline 16 & 5.186 & 3.6 & 4.8 & 5.3 & 5.7 & 6.2 & 81.858 \\
\hline 17 & 7.110 & 9.3 & 6.0 & 6.5 & 7.1 & 6.2 & 88.401 \\
\hline 18 & 5.356 & 3.7 & 4.5 & 5.2 & 6.0 & 6.8 & 84.992 \\
\hline 19 & 5.210 & 4.1 & 4.6 & 5.3 & 5.6 & 6.2 & 84.526 \\
\hline 20 & 6.889 & 6.7 & 6.6 & 7.1 & 6.9 & 7.1 & 82.084 \\
\hline Mean & $\mathbf{6 . 0 4 3}$ & $\mathbf{4 . 9 0 5}$ & $\mathbf{5 . 5 4 5}$ & $\mathbf{6 . 1 9 0}$ & $\mathbf{6 . 5 7 5}$ & $\mathbf{6 . 6 9 5}$ & $\mathbf{8 7 . 4 0 7}$ \\
\hline SD & 1.145 & $\mathbf{1 . 5 7 8}$ & $\mathbf{1 . 2 2 8}$ & $\mathbf{1 . 2 6 1}$ & $\mathbf{1 . 3 7 0}$ & $\mathbf{0 . 8 7 1}$ & $\mathbf{6 . 5 2 8}$ \\
\hline Min & $\mathbf{5 . 1 8 6}$ & $\mathbf{3 . 5 0 0}$ & $\mathbf{4 . 5 0 0}$ & $\mathbf{5 . 2 0 0}$ & $\mathbf{5 . 4 0 0}$ & $\mathbf{5 . 7 0 0}$ & $\mathbf{8 1 . 6 4 9}$ \\
\hline Max & $\mathbf{8 . 8 5 2}$ & $\mathbf{9 . 3 0 0}$ & $\mathbf{8 . 7 0 0}$ & $\mathbf{9 . 6 0 0}$ & $\mathbf{1 0 . 4 0 0}$ & $\mathbf{9 . 1 0 0}$ & $\mathbf{1 0 8 . 2 1 4}$ \\
\hline RMSE/Mean & $\mathbf{0 . 0 0 7 0}$ & $\mathbf{0 . 0 0 5 7}$ & $\mathbf{0 . 0 0 6 4}$ & $\mathbf{0 . 0 0 7 1}$ & $\mathbf{0 . 0 0 7 6}$ & $\mathbf{0 . 0 0 7 7}$ & \\
\hline & & & & & & & \\
\hline
\end{tabular}

Figure 45: The CNN Model \#2 - (a) the model architecture, and (b) regression results of the univariate time series model with the previous two weeks' data as the input

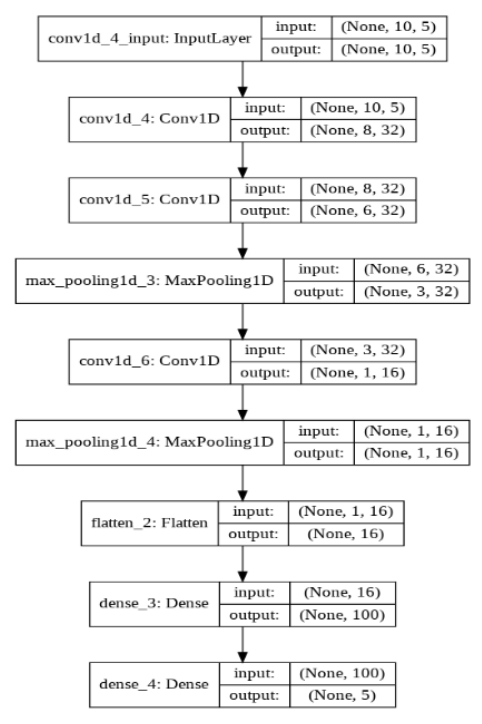

\begin{tabular}{|c|c|c|c|c|c|c|c|}
\hline Round_No & Overall RMSE & Monday & Tuesday & Wednesday & Thursday & Friday & Exec_Time \\
\hline 1 & 8.466 & 7.9 & 6.7 & 7.4 & 9.6 & 10.1 & 134.286 \\
\hline 2 & 5.510 & 4.2 & 5.2 & 5.6 & 6.1 & 6.3 & 122.020 \\
\hline 3 & 6.003 & 4.7 & 5.6 & 6.3 & 6.4 & 6.8 & 111.729 \\
\hline 4 & 6.718 & 5.6 & 6.4 & 7.0 & 7.2 & 7.3 & 116.582 \\
\hline 5 & 5.602 & 4.4 & 5.2 & 5.7 & 6.1 & 6.4 & 131.704 \\
\hline 6 & 6.056 & 4.3 & 5.9 & 6.2 & 6.7 & 6.8 & 113.107 \\
\hline 7 & 5.585 & 4.3 & 5.0 & 5.7 & 5.9 & 6.8 & 137.406 \\
\hline 8 & 6.220 & 4.6 & 6.6 & 6.5 & 6.4 & 6.8 & 113.899 \\
\hline 9 & 5.710 & 4.5 & 5.4 & 5.9 & 6.1 & 6.4 & 113.717 \\
\hline 10 & 6.101 & 5.1 & 6.0 & 6.2 & 6.3 & 6.7 & 111.018 \\
\hline 11 & 6.708 & 6.7 & 6.6 & 6.5 & 6.6 & 7.1 & 131.253 \\
\hline 12 & 5.398 & 4.1 & 5.0 & 5.5 & 5.9 & 6.2 & 139.208 \\
\hline 13 & 7.956 & 8.3 & 6.4 & 6.4 & 7.8 & 10.2 & 114.197 \\
\hline 14 & 7.061 & 6.5 & 6.3 & 6.4 & 8.4 & 7.5 & 115.814 \\
\hline 15 & 5.870 & 4.5 & 5.6 & 6.1 & 6.4 & 6.5 & 116.391 \\
\hline 16 & 6.070 & 4.7 & 5.7 & 6.2 & 6.5 & 7.0 & 113.899 \\
\hline 17 & 5.977 & 5.2 & 5.4 & 6.0 & 6.4 & 6.7 & 116.089 \\
\hline 18 & 5.760 & 4.8 & 5.0 & 6.5 & 6.1 & 6.2 & 114.035 \\
\hline 19 & 5.647 & 4.9 & 5.2 & 5.7 & 6.0 & 6.3 & 112.558 \\
\hline 20 & 5.547 & 4.4 & 5.1 & 5.6 & 5.9 & 6.5 & 116.758 \\
\hline Mean & $\mathbf{6 . 1 9 8}$ & $\mathbf{5 . 1 8 5}$ & $\mathbf{5 . 7 1 5}$ & $\mathbf{6 . 1 7}$ & $\mathbf{6 . 6 4}$ & $\mathbf{7 . 0 3}$ & $\mathbf{1 1 9 . 7 8 4}$ \\
\hline SD & $\mathbf{0 . 8 1 9}$ & $\mathbf{1 . 2 1 8}$ & $\mathbf{0 . 6 0 1}$ & $\mathbf{0 . 4 8 8}$ & $\mathbf{0 . 9 4 9}$ & $\mathbf{1 . 1 2 4}$ & $\mathbf{9 . 3 0 7}$ \\
\hline Min & $\mathbf{5 . 3 9 8}$ & $\mathbf{4 . 1}$ & $\mathbf{5}$ & $\mathbf{5 . 5}$ & $\mathbf{5 . 9}$ & $\mathbf{6 . 2}$ & $\mathbf{1 1 1 . 0 1 8}$ \\
\hline Max & $\mathbf{8 . 4 6 6}$ & $\mathbf{8 . 3}$ & $\mathbf{6 . 7}$ & $\mathbf{7 . 4}$ & $\mathbf{9 . 6}$ & $\mathbf{1 0 . 2}$ & $\mathbf{1 3 9 . 2 0 8}$ \\
\hline RMSE/Mean & $\mathbf{0 . 0 0 7 2}$ & $\mathbf{0 . 0 0 6 0}$ & $\mathbf{0 . 0 0 6 6}$ & $\mathbf{0 . 0 0 7 1}$ & $\mathbf{0 . 0 0 7 7}$ & $\mathbf{0 . 0 0 8 1}$ & \\
\hline & & & & & &
\end{tabular}

Figure 46: The CNN Model \#3 - (a) the model architecture, and (b) regression results of the multivariate time series model with the previous two weeks' data as the input

CNN Model \#III: This model is based on a multivariate forecasting approach with the previous two week's data as the input. In this case, the multi-channel CNN model uses all the values of the variables - open, high, low, close, and volume - over the previous two weeks for forecasting the next week's open values. This is done by allowing each variable of the time series to enter into the model as a separate channel of input. In this case, CNN uses a separate kernel, and reads each input sequence into a separate set of features, and learning from those features. The increase in the amount of data requires a larger and more sophisticated model that is trained for a longer time. Two convolutional layers, each with 32 filter maps and a kernel size of 3 are used. The two convolutional layers are followed by a max-pooling layer of size 2 . The output of 
the max-pooling layer is passed onto a third convolutional layer with 16 filter maps with a kernel size of 3. The third convolutional layer passes its output to a max-pooling layer of size 1. A fully connected layer with 100 nodes then interprets the features before finally sending the predicted values to the five nodes in the output layer. The model is trained over 70 epochs with a batch size of 16 . The activation function for all layers is ReLU, and the Adam optimizer is used for optimizing the stochastic gradient descent algorithm. Fig. 46(a) depicts the architecture of the CNN Model \#3. The performance results of the model are presented in Fig. 46(b).

From Figs. 44, 45, and 46, it is evident observed that the CNN Model \#1 - the univariate CNN model with the previous one week's data as the input is the most accurate. It yields a ratio of RMSE to the mean of the actual values as 0.0065 . This model is also found to be the fastest in execution with a mean execution time of 82.668 seconds for 20 rounds. The CNN Model \#III - the multivariate CNN model with the previous two weeks' data as the input, is found to have performed the worst. This model yields a value of 0.0072 for the ratio of the RMSE to the mean of the actual values. The model also takes the longest time to execute. The mean execution time per round for this model is found to be 119.784 seconds for the 20 rounds over which the model is trained. It is also noted that for all the models, the ratio of RMSE to the mean value of the response variable is the lowest for Monday, and the same is the highest for Friday. The ratio of RMSE to the mean open value consistently increased from Monday through Friday.

We now present a summary of the performance results for all the machine learning-based classification and regression results.

Overall Performance: Finally, we summarize the performance of the machine learning and LSTM models different predictive models. Tables $21-23$ present the performance of the classification models for Case I, Case II, and Case III respectively. For each case and each metric, the best model is marked with a bold font.

Table 21: Summary of the performance of the classification models in Case I

\begin{tabular}{|l|c|c|c|c|c|c|c|c|}
\hline & LR & KNN & DT & BAG & BOOST & RF & ANN & SVM \\
\hline Sensitivity & 94.79 & 89.57 & 95.09 & 95.09 & $\mathbf{1 0 0 . 0 0}$ & 94.48 & 95.40 & 94.46 \\
\hline Specificity & 97.61 & 96.42 & 98.09 & 98.09 & $\mathbf{1 0 0 . 0 0}$ & 97.61 & 97.61 & 95.58 \\
\hline PPV & 96.87 & 95.11 & 97.48 & 97.48 & $\mathbf{1 0 0 . 0 0}$ & 96.86 & 96.88 & 94.17 \\
\hline NPV & 96.01 & 92.24 & 96.25 & 96.25 & $\mathbf{1 0 0 . 0 0}$ & 98.08 & 96.46 & 98.09 \\
\hline CA & 96.38 & 93.42 & 96.78 & 96.78 & $\mathbf{1 0 0 . 0 0}$ & 96.24 & 96.64 & 96.38 \\
\hline F1 & 95. & 92. & 96. & 96.0 & $\mathbf{1 0 0 . 0 0}$ & 95.6 & 96. & 94.3 \\
Score & 82 & 26 & 27 & 7 & & 6 & 13 & 1 \\
\hline
\end{tabular}

Table 22: Summary of the performance of the classification models in Case II

\begin{tabular}{|l|c|c|c|c|c|c|c|c|}
\hline & LR & KNN & DT & BAG & BOOST & RF & ANN & SVM \\
\hline Sensitivity & 94.83 & 86.93 & 92.40 & 95.44 & $\mathbf{1 0 0 . 0 0}$ & 93.01 & 93.62 & 94.67 \\
\hline Specificity & 95.96 & 92.93 & 95.71 & 96.46 & $\mathbf{1 0 0 . 0 0}$ & 94.19 & 95.71 & 93.35 \\
\hline PPV & 95.12 & 91.08 & 94.70 & 95.73 & $\mathbf{1 0 0 . 0 0}$ & 93.01 & 94.77 & 91.79 \\
\hline NPV & 95.72 & 89.54 & 93.81 & 96.22 & $\mathbf{1 0 0 . 0 0}$ & 94.19 & 94.75 & 95.71 \\
\hline CA & 95.45 & 90.21 & 94.21 & 96.00 & $\mathbf{1 0 0 . 0 0}$ & 93.66 & 94.76 & 93.93 \\
\hline F1 Score & 94.97 & 88.96 & 93.54 & 95.58 & $\mathbf{1 0 0 . 0 0}$ & 93.01 & 94.19 & 93.21 \\
\hline
\end{tabular}

We observe that both for Case I and Case II and all the metrics, boosting performed the best among all the classification models. However, because Case I and Case II represent only the training scenarios, the performance in Case III that depicts the performance in the testing phase should be considered as the most critical. From Table 23, it is observed that the ANN model performs the best on sensitivity and NPV, while 
the boosting model outperforms all other models on specificity, PPV, and classification accuracy. However, the random forest model is found to have performed best on the Fl score - the metric that is usually considered to be the most important one in classification. In Tables 21-26, the following abbreviations are used in the column names: $\mathrm{LR}$ - logistic regression, $\mathrm{KNN}$ - k-nearest neighbor, DT- decision tree, $\mathrm{BAG}-$ bagging, BOOST - boosting, RF - random forest, ANN - artificial neural networks, SVM - support vector machines, MV - multivariate regression, MARS - multivariate adaptive regression spline, LSTM - longand-short- term memory.

Table 23: Summary of the performance of the classification models in Case III

\begin{tabular}{|l|c|c|c|c|c|c|c|c|}
\hline & LR & KNN & DT & BAG & BOOST & RF & ANN & SVM \\
\hline Sensitivity & 92.10 & 84.50 & 89.97 & 89.97 & 92.10 & 91.19 & $\mathbf{9 9 . 7 0}$ & 93.81 \\
\hline Specificity & 89.39 & 48.99 & 92.42 & 92.42 & $\mathbf{9 3 . 4 3}$ & 92.93 & 34.60 & 90.19 \\
\hline PPV & 87.83 & 57.92 & 90.80 & 90.78 & $\mathbf{9 2 . 1 0}$ & 91.46 & 55.88 & 87.54 \\
\hline NPV & 93.16 & 79.18 & 91.73 & 91.73 & 93.43 & 92.70 & $\mathbf{9 9 . 2 8}$ & 95.20 \\
\hline CA & 90.62 & 65.10 & 91.31 & 91.31 & $\mathbf{9 2 . 8 3}$ & 92.14 & 64.14 & 91.72 \\
\hline F1 Score & 89.91 & 68.73 & 90.38 & 90.37 & 92.10 & $\mathbf{9 1 . 3 2}$ & 71.62 & 90.57 \\
\hline
\end{tabular}

Table 24: Summary of the performance of the regression models in Case I

\begin{tabular}{|l|c|c|c|c|c|c|c|c|c|}
\hline & MV & MARS & DT & BAG & BOOST & RF & ANN & SVM & LSTM \\
\hline Correlation & $\mathbf{0 . 9 9}$ & $\mathbf{0 . 9 9}$ & 0.97 & 0.96 & $\mathbf{0 . 9 9}$ & $\mathbf{0 . 9 9}$ & $\mathbf{0 . 9 9}$ & 0.93 & $\mathbf{1 . 0 0}$ \\
\hline RMSE/Mean & 13.32 & $\mathbf{1 2 . 4 1}$ & 35.35 & 40.29 & 23.40 & 16.26 & 17.16 & 53.88 & $\mathbf{7 . 9 4}$ \\
\hline Mismatched Cases & 18.67 & 1.21 & 13.42 & 4.70 & 0.81 & $\mathbf{0 . 0 0}$ & 1.21 & $\mathbf{0 . 2 7}$ & $\mathbf{0 . 0 0}$ \\
\hline
\end{tabular}

Table 25: Summary of the performance of the regression models in Case II

\begin{tabular}{|l|c|c|c|c|c|c|c|c|c|}
\hline & MV & MARS & DT & BAG & BOOST & RF & ANN & SVM & LSTM \\
\hline Correlation & $\mathbf{0 . 9 9}$ & $\mathbf{0 . 9 9}$ & 0.97 & 0.98 & $\mathbf{0 . 9 9}$ & $\mathbf{0 . 9 9}$ & 0.98 & 0.98 & $\mathbf{1 . 0 0}$ \\
\hline RMSE/Mean & 18.84 & 17.09 & 37.04 & 25.70 & 17.35 & $\mathbf{1 0 . 8 2}$ & 31.39 & 27.92 & $\mathbf{4 . 0 4}$ \\
\hline Mismatched Cases & 5.38 & 4.28 & 17.38 & 5.10 & 4.69 & $\mathbf{2 . 6 2}$ & 9.38 & 4.41 & $\mathbf{0 . 0 0}$ \\
\hline
\end{tabular}

Table 26: Summary of the performance of the regression models in Case III

\begin{tabular}{|l|c|c|c|c|c|c|c|c|c|}
\hline & MV & MARS & DT & BAG & BOOST & RF & ANN & SVM & LSTM \\
\hline Correlation & $\mathbf{0 . 9 9}$ & $\mathbf{0 . 9 9}$ & 0.10 & 0.97 & 0.97 & 0.97 & 0.98 & 0.83 & $\mathbf{0 . 9 9}$ \\
\hline RMSE/Mean & $\mathbf{1 8 . 8 8}$ & 20.40 & 165.92 & 34.91 & 41.51 & 32.02 & 36.83 & 82.96 & $\mathbf{2 . 3 6}$ \\
\hline Mismatched Cases & $\mathbf{5 . 2 4}$ & 6.34 & 47.72 & 9.24 & 6.90 & 6.48 & 10.90 & 13.19 & $\mathbf{2 . 4 0}$ \\
\hline
\end{tabular}

Tables $24-26$ present the performance results of the regression models, including the LSTM-based deep learning model. Since the LSTM model outperforms all the machine learning models on all the metrics and for all three cases, we also identify the best performing machine learning model on each metric.

In Case I, multivariate regression, MARS, boosting, random forest, and ANN all yield the highest correlation coefficient value of 0.99 . However, the correlation coefficient is found to be 1.00 in the case of LSTM. For the ratio of the RMSE to the mean of the absolute values of the open perc values, MARS yields the lowest value of 12.41 among the machine learning models, while the corresponding value for LSTM is 7.94. Both random forest and LSTM yield no sign mismatch between the predicted and the actual values of open_perc. 
In Case II, the highest value of the correlation coefficient is achieved by multivariate regression, MARS, boosting, and random forest. LSTM outperforms all the machine learning models on this metric by attaining a value of 1.00 . The RMSE to the mean ratio value of 10.82 is the least for random forest among the machine learning models. However, the corresponding value yielded by LSTM was 4.04. Random forest produces only 2.62 percent cases that mismatch in the signs of the actual and the predicted open perc values, however for LSTM, all the cases have the same sign for the actual and the predicted open_perc values.

For Case III, while LSTM exhibits the best performance on all metrics, multivariate regression and MARS yield the same (the highest) value for the correlation coefficient. For the metric RMSE to the mean ratio, and the percentage of the mismatched cases, multivariate regression produces the best results among the machine learning models.

It may be noted that the CNN models are built and tested on the stock price data from December 31, 2012, to January 9, 2015, and the data are of 5 minutes' granularity. The predictions are made on one week's time horizon daily. Since all other models built in this work are based on stock price data aggregated into three slots in a day, it is not wise to compare the performance of the CNN model suites with the machine learningbased models and the LSTM models. However, one can easily see that based on the ratio of the RMSE to the mean of the actual values of the forecasted variable, all the CNN models outperform the LSTM by a large margin. While the least value for the ratio of the RMSE to the actual value of the forecasted variable for the LSTM model was found to be 2.36, the corresponding value for the CNN suite was 0.0065 .

\section{Conclusion and Future Work}

In this paper, we propose a robust forecasting framework for stock price and stock price movement patterns with a very high level of accuracy. The predictive model consists of eight classification and eight regression models based on several machine learning approaches. Also, the predictive framework includes two deep learning models of regression built on an LSTM network and a suite of CNNs. The machine learning-based models and the LSTM model use stock price data of three slots in a day and predict the stock price slotwise over a prediction horizon of one year. We construct the models, train, validate, and finally test them using the historical stock prices of a company - Godrej Consumer Products Ltd. The data is extracted from the listed values of the stock in the National Stock Exchange (NSE) of India during the period of two years - January 2013 till December 2014. The stock price data are extracted at five minutes intervals of time using the Metastock tool. The raw data is pre-processed, appropriate transformation (i.e., normalization, standardization, NA removal, etc.) done, and several derived predictor variables are created based on the rich features of the stock data. While several newly derived predictors are used in building the model, we use the percentage change in the open values of the stock, called open_perc, as the response variable. The five minutes interval granular data are also aggregated into three slots on a given day so that the predictive models can be built to forecast the value of the open_perc in the next slot given stock price data till the current slot. While the classification-based models are used to predict the movement pattern of open_perc values, the objective of the regression models is to accurately predict the value of the open_perc. In addition to exploiting the machine learning algorithms for building the eight classification and eight regression models, we also leverage the rich features of Tensorflow and Keras frameworks in building two extremely powerful deep learning-based regression models using an LSTM network and a suite of CNNs. For building the machine learning models, we use the R programming language, while for the LSTM-based deep 
learning regression model, and the suite of four CNN models, the Python programming has been used. The results elicit a very interesting observation. While no single machine learning model performs the best on all the metrics on classification and regression, the deep learning model using an LSTM network outperforms all the machine learning-based regression models on every metric. Since the CNN models are built using stock price data collected at 5 minutes interval of time while the machine learning models and the LSTM models are based on stock price data collected at three slots in a day, it is not appropriate to compare the performance of the CNN suite with the other models. However, it has been found that based on the metric of the ratio of the RMSE to the mean of the actual values of the forecasted variable, the CNN models are far more accurate than the machine learning models and the LSTM-based deep learning models.

\section{Reference}

Ariyo, A. A., Adewumi, A. O. and Ayo, C. K. (2014) 'Stock price prediction using the ARIMA model', $16^{\text {th }}$ International Conference on Computer Modelling and Simulation (UKSIM'14), Cambridge, UK, 26-28 March, pp. 106 - 112. DOI:10.1109/UKSim.2014.67.

Asghar, M. Z., Rahman, F., Kundi, F. M. and Ahmed, S. (2019) 'Development of stock market trend prediction system using multiple regression', Computational and Mathematical Organization Theory, Vol. 25, pp. 271-301, DOI: 10.1007/s10588-019-09292-7.

Basalto, N., Bellotti, R., De Carlo, F., Facchi, P. and Pascazio, S. (2005) 'Clustering stock market companies via chaotic map synchronization', Physica A: Statistical Mechanics and its Applications, Vol. 345, Nos. 1 - 2, pp. 196-206, DOI: 10.1016/j.physa.2004.07.034.

Bao, W., Yue, J. and Rao, Y. (2017) 'A deep learning framework for financial time series using stacked autoencoders and long-and-short-term memory', PLOSE ONE, Vol. 12, No. 7, DOI: 10.1371/journal.pone.0180944

Bentes, S. R., Menezes, R. and Mendes, D. A. (2008) 'Long memory and volatility clustering: Is the empirical evidence consistent across stock markets?', Physica A: Statistical Mechanics and its Applications, Vol. 387, No. 15, pp. 3826-3830, DOI: 10.1016/j.physa.2008.01.046.

Binkowski, M., Marti, G. and Donnat, P. (2018) 'Autoregressive convolutional neural networks for asynchronous time series', 35 ${ }^{\text {th }}$ International Conference on Machine Learning (ICML'18), 10-15 July, Sydney, Australia.

Chen, A.-S., Leung, M. T. and Daouk, H. (2003) 'Application of neural networks to an emerging financial market: forecasting and trading the Taiwan Stock Index', Computers and Operations Research, Vol. 30, No. 6, pp. 901-923, DOI: 10.1016/S0305-0548(02)00037-0.

Chen, Y., Dong, X. and Zhao, Y. (2005) 'Stock index modeling using EDA based local linear wavelet neural network', International Conference on Neural Networks and Brain (ICNNB'15), Beijing, China, 13 - 15 October, pp. 1646 - 1650, DOI: 10.1109/ICNNB.2005.1614946.

de Faria, E. L., Albuquerque, M. P., Gonzalez, J. L., Cavalcante, J. T. P. and Albuquerque, M. P. (2009) 'Predicting the Brazilian stock market through neural networks and adaptive exponential smoothing methods', Expert Systems with Applications, Vol. 36, No. 10, pp. 12506-12509, DOI: 10.1016/j.eswa.2009.04.032.

Dutta, G., Jha, P., Laha, A. K. and Mohan, N. (2006) 'Artificial neural network models for forecasting stock price index in the Bombay Stock Exchange', Journal of Emerging Market Finance, Vol 5, No 3, pp. 283295, DOI: $10.1177 / 097265270600500305$. 
Enke, D., Grauer, M. and Mehdiyev, N. (2011) 'Stock market prediction with multiple regression, fuzzy type-2 clustering, and neural networks', Procedia Computer Science, Vol 6, pp. $201-206$.

Fu, T-C, Chung, F-L., Luk, R. and Ng, C-M, (2008) 'Representing financial time series based on data point importance', Engineering Applications of Artificial Intelligence, Vol. 21, No. 2, pp. 277-300, DOI: 10.1016/j.engappai.2007.04.009.

Geron, A. (2019). Hands-on Machine Learning with Scikit-Learn Keras \& Tensorflow. O'Reilly Media Inc., 2nd Edition, September, 2019, Sebastopol, CA, USA. ISBN: 9781492032632.

Hammad, A. A. A., Ali, S. M. A. and Hall, E. L. (2007) 'Forecasting the Jordanian stock prices using artificial neural networks', Book Chapter in the book: Intelligent Engineering Systems through Artificial Neural Networks, ASME Press, NY, USA.

Hanias, M., Curtis, P. and Thalassinos, E. (2012) 'Time series prediction with neural networks for the Athens Stock Exchange indicator', European Research Studies Journal, Vol. 15, No. 2, pp. 23-32, DOI: $10.35808 / \mathrm{ersj} / 351$.

Ivanovski, Z., Ivanovska, N. and Narasanov, Z. (2016) 'The regression analysis of stock returns at MSE', Journal of Modern Accounting and Auditing, Vol. 12, No. 4, pp. 217-224.

Jammalamadaka, S. R., Qui, J. and Ning, N. (2019) 'Predicting a stock portfolio with multivariate Bayesian structural time series model: Do news or emotions matter?', International Journal of Artificial Intelligence, Vol. 17, No. 2, pp. 81-104.

Jarrett, J. E. and Kyper E. (2011) 'ARIMA modeling with intervention to forecast and analyze Chinese stock prices', International Journal of Engineering Business Management, Vol. 3, pp. 53-58, DOI: $10.5772 / 50938$.

Khan, U., Aadil, F., Ghazanfar, M., Khan, S., Metawa, N., Muhammad, K., Mehmood, I. and Nam, Y. (2018) 'A robust regression-based stock exchange forecasting and determination of correlation between stock markets', Sustainability, Vol. 10, No. 3702, pp. 20. DOI: 10.3390/su10103702.

Lahmiri, S. (2014) 'Wavelet low- and high- frequency components as features for predicting stock prices with backpropagation Neural Networks', Journal of King Saud University - Computer and Information Sciences, Vol 26, No 2, pp. 218-227. DOI: 10.1016/j.jksuci.2013.12.001.

Leigh, W., Hightower, R. and Modani, N. (2005) 'Forecasting the New York stock exchange composite index with past price and interest rate on condition of volume spike', Expert Systems with Applications, Vol. 28, No. 1, pp. 1-8, DOI: 10.1016/j.eswa.2004.08.001.

Liao, S-H., Ho, H-H., Lin and H-W. (2008) 'Mining stock category association and cluster on Taiwan stock market', Expert System with Applications, Vol. 35, No. 1-2, pp. 19-29, DOI: 10.1016/j.eswa.2007.06.001.

Ma, J. and Liu, L. (2008) 'Multivariate nonlinear analysis and prediction of Shanghai stock Market', Discrete Dynamic in Nature and Society, Vol. 2008, May, pp. 1-8, Article ID: 526734. DOI: $10.1155 / 2008 / 526734$.

Mehtab, S., Sen, J. (2021a). 'Analysis and forecasting of financial time series using CNN and LSTM-based deep learning models', In: Proceedings of the $2^{\text {nd }}$ International Conference on Advances in Distributed Computing and Machine Learning (ICADCMl'21), January 15-15, 2021, Bhubaneswar, India. (Accepted for publication)

Mehtab, S. and Sen, J. (2020a) 'Stock price prediction using convolutional neural networks on a multivariate time series', Proceedings of the 3rd National Conference on Machine Learning and Artificial Intelligence (NCMLAI'20), February 1, New Delhi, India. 
Mehtab, S. and Sen J. (2020b) Time Series Analysis-Based Stock Price Prediction Framework Using Machine Learning and Deep Learning Models. Technical Report No: NSHM_KOL_2020_SCA_DS_1, 2020. DOI: $10.13140 /$ RG.2.2.14022.22085/2.

Mehtab, S. and Sen, J. (2020c) 'Stock price prediction using CNN and LSTM-based deep learning models', Proceedings of the IEEE International Conference on Decision Aid Sciences and Applications (DASA'20), November 8-9, 2020, Sakheer, Bahrain, pp. 447-453. DOI: 10.1109/DASA51403.2020.9317207.

Mehtab, S., Sen, J., and Dasgupta, S. (2020d) 'Robust analysis of stock price time series using CNN and LSTM-based deep learning models', Proceedings of the $4^{\text {th }}$ IEEE International Conference on Electronics, Communication and Aerospace Technology (ICECA'20), November 5-7, 2020, Coimbatore, India, pp. 1481-1486. DOI: 10.1109/ICECA49313.2020.9297652.

Mehtab, S. Sen, J., and Dutta, A. (2020e) 'Stock price prediction using machine learning and LSTM-based deep learning models', In: Thampi, S. M., Piramuthu, S., Li, K. C., Beretti, S., Wozniak, M., Singh, D. (eds.), Machine Learning and Metaheuristics Algorithms and Applications, SoMMA 2020. Communications in Computer and Information Science, Vol 1366, pp. 88-106, Springer, Singapore. DOI: 10.1007/978-981-16-0419-5 8.

Mehtab, S. and Sen, J. (2019) 'A robust predictive model for stock price prediction using deep learning and natural language processing', Proceedings of the 7th International Conference on Business Analytics and Intelligence (BAICONF'19), Bangalore, India, 5-7 December, DOI: 10.2139/ssrn.3502624.

Metastock Website: http://www.metastock.com (accessed May 28 2021)

Mondal, P., Shit, L. and Goswami, S. (2014) 'Study of effectiveness of time series modeling (ARMA) in forecasting stock prices', International Journal of Computer Science, Engineering and Applications, Vol. 4, No. 2, pp. 13-29, DOI: 10.5121/ijcsea.2014.4202.

Moshiri, S. and Cameron, N. (2010) 'Neural network versus econometric models in forecasting inflation', Journal of Forecasting, Vol. 19, No. 3, pp. 201-217. DOI: 10.1002/(SICI)1099131X(200004)19:3<201::AID-FOR753>3.0.CO;2-4.

Mostafa, M. M. (2010) 'Forecasting stock exchange movements using neural networks: Empirical evidence from Kuwait', Expert Systems with Application, Vol. 37, No. 9, pp. 6302-6309, DOI: 10.1016/j.eswa.2010.02.091.

Ning, B., Wu, J., Peng, H. and Zhao, J. (2009) 'Using chaotic neural network to forecast stock index', In: Yu, W., He, H., Zhang, N. (eds) Advances in Neural Networks - ISNN 2009, Lecture Notes in Computer Science, Vol. 551, pp. 870-876, Springer, Berlin, Heidelberg, DOI: 10.1007/978-3-642-01597-6_98.

Ning, Y., Wah, L. C. and Erdan, L. (2019) 'Stock price prediction based on error correction model and Granger causality test', Cluster Computing, Vol. 22, pp. 4849-4858, 2019, DOI: 10.1007/s10586-0182406-6.

Obthoong, M., Tantisantiwong, N., Jeamwatthanachai, W. and Will, G. (2020) 'A survey on machine learning for stock price prediction: Algorithms and techniques', Proceedings of the 2nd International Conference on Finance, Economics, Management and Business (IT FEMIB'20), May 5 -6, Prague, Czech Republic.

Park, N. J., George, K. M. and Park, N. (2010) 'A multiple regression model for trend change prediction', Proceedings of the 2010 IEEE International Conference on Financial Theory and Engineering, 18-20 June, Dubai, USE, DOI: 10.1109/CFTE.2010.5499430.

Phua, P. K. H., Ming, D. and Lin, W. (2001) 'Neural network with genetically evolved algorithms for stock prediction', Asia-Pacific Journal of Operational Research, Vol. 18, No. 1, pp. 103 - 107. 
Porshnev, A., Redkin, I. and Shevchenko, A. (2013) Machine learning in prediction of stock market indicators based on historical data and data from Twitter sentiment analysis', Proceedings of the IEEE International Conference on Data Mining Workshops, Dallas, TX, USA.

Sen, J., Mehtab, S. (2021a). 'Accurate stock price forecasting using robust and optimized deep learning models', Proceedings of the IEEE International Conference on Intelligent Computing (CONIT'21), June 25-27, 2021, Hubli, India. (Accepted for publication)

Sen, J., Dutta, A., and Mehtab, S. (2021b). 'Profitability analysis in stock investment using an LSTM-based deep learning model', Proceedings of the $2^{\text {nd }}$ IEEE International Conference on Emerging Technologies (INCET'21), May 22-23, 2021, Belgaum, India. (Accepted for publication)

Sen, J. and Datta Chaudhuri, T. (2018a) 'Understanding the sectors of Indian economy for portfolio choice', International Journal of Business Forecasting and Marketing Intelligence, Vol. 4, No. 2, pp. 178-222, DOI: 10.1504/IJBFMI.2018.090914.

Sen, J. (2018b) 'Stock composition of mutual funds and fund style: A time series decomposition approach towards testing for consistency', International Journal of Business Forecasting and Marketing Intelligence, Vol. 4, No. 3, pp. 235-292, DOI: 10.1504/IJBFMI.2018.092781.

Sen, J. (2018c) 'A study of the Indian metal sector using time series decomposition-based approach', Book Chapter in Selected Studies on Economics and Finance, Editors: Basar, S., Celik, A. A., \& Bayramoglu, T., pp. 105-152, Cambridge Scholars Publishing, UK, March 2018.

Sen, J. (2018d) 'Stock price prediction using machine learning and deep learning frameworks', 6th International Conference on Business Analytics and Intelligence (ICBAI'18), 20-22 December, Bangalore, India.

Sen, J. and Datta Chaudhuri, T. (2017) 'A predictive analysis of the Indian FMCG sector using time series decomposition-based approach', Journal of Economics Library, Vol. 4, No. 2, pp. 206-226, DOI: 10.1453/jel.v4i2.1282.

Sen, J. and Datta Chaudhuri, T. (2016a) 'Decomposition of time series data of stock markets and its implications for prediction -An application for the Indian auto sector', 2nd National Conference on Advances in Business Research and Management Practices (ABMRP'16), Kolkata, India, 8-9 January, pp, 1-28, DOI: 10.13140/RG.2.1.3232.0241.

Sen, J. and Datta Chaudhuri, T. (2016b) 'Decomposition of time series data to check consistency between fund style and actual fund composition of mutual funds', 4th International Conference on Business Analytics and Intelligence (ICBAI'16), Bangalore, India, 19-21 December, DOI: 10.13140/RG.2.2.33048.19206.

Sen, J. and Datta Chaudhuri, T. (2016c) 'An alternative framework for time series decomposition and forecasting and its relevance for portfolio choice - A comparative study of the Indian consumer durable and small-cap sector', Journal of Economics Library, Vol. 3, No. 2, pp. 303-326, DOI: 10.1453/jel.v3i2.787.

Senol, D. and Ozturan, M. (2008) 'Stock price direction prediction using artificial neural network approach: The case of Turkey', Journal of Artificial Intelligence, Vol. 1, No. 2, pp. 70-77, DOI: 10.3923/jai.2008.70.77

Shen, J., Fan, H. and Chang, S. (2007) 'Stock index prediction based on adaptive training and pruning algorithm', Advances in Neural Networks, Lecture Notes in Computer Science, Springer-Verlag, Vol 4492, pp. 457-464, DOI. 10.1007/978-3-540-72393-6 55.

Tang, J. and Chen, X. (2018) 'Stock market prediction based on historic prices and news titles', Proceedings of the International Conference on Machine Learning Technologies (ICMLT'18), Jinan, China, pp. 2934. 
Tseng, K-C., Kwon, O. and Tjung, L. C. (2012) 'Time series and neural network forecast of daily stock prices’, Investment Management and Financial Innovations, Vol. 9, No. 1, pp. 32-54.

Vantuch, T. and Zelinka, I. (2014) 'Evolutionary based ARIMA models for stock price forecasting', Proceedings of the Interdisciplinary Symposium on Complex Systems (ISCS'14), pp. 239-247, DOI: 10.1007/978-3-319-10759-2_25.

Vargas, M. R., de Lima, B. S. L. P. and Evsukoff, A. G. (2017) 'Deep learning for stock market prediction from financial news article', Proceedings of the IEEE International Conference on Computational Intelligence and Virtual Environments for Measurement Systems and Applications (CIVEMSA), 31 July, Annecy, France, DOI: 10.1109/CIVEMSA.2017.7995302.

Wang, Z., Ho, S-B. and Lin, Z. (2018) 'Stock market prediction analysis by incorporating social and news opinion and sentiment', Proceedings of the IEEE International Conference on Data Mining Workshops, Singapore.

Wu, Q., Chen, Y. and Liu, Z. (2008) 'Ensemble model of intelligent paradigms for stock market forecasting', $1^{\text {st }}$ International Workshop on Knowledge Discovery and Data Mining (WKDD’08), Washington, DC, USA, pp. 205 - 208, DOI: 10.1109/WKDD.2008.54.

Xiao, Y., Xiao, J., Liu, J. and Wang, S. (2014) 'A multiscale modeling approach incorporating ARIMA and ANNs for financial market volatility forecasting', Journal of Systems Science and Complexity, Vol. 27, No, 1, pp. 225-236.

Yan, Z., Huang, Z. and Liang, M. (2019) 'Stock prediction via linear regression and BP regression network', Communications in Mathematical Finance, Vol. 8, N0. 1, pp. 1-20.

Zhang, D., Jiang, Q. and Li, X. (2007) 'Application of neural networks in financial data mining', International Journal of Computer, Electrical, Automation, and Information Engineering, Vol. 1, No. 1, pp. 225-228, DOI: 10.5281/zenodo.1333234.

Zhou, J. and Fan, P. (2019) 'Modulation format/bit rate recognition based on principal component analysis (PCA) and artificial neural networks (ANNs)', OSA Continuum, Vol. 2, No. 3, pp. 923-937.

Zhu, X., Wang, H., Xu, L. and Li, H. (2008) 'Predicting stock index increments by neural networks: The role of trading volume under different horizons', Expert Systems Applications, Vol. 34, No. 4, pp. 30433054. DOI: 